Supporting Information for:

\title{
One-Pot Regiodirected Annulations for the Rapid Synthesis of $\pi$-Extended Oligomers
}

Andrea Nitti, Peshawa Osw, Giuseppe Calcagno, Chiara Botta, Samuel I. Etkind, Gabriele Bianchi, Riccardo Po, Timothy M. Swager and Dario Pasini*

\section{Table of Contents}

1. General experimental $\quad S 2$

2. Synthesis of known compounds $\quad S 2$

2. Synthesis of new compounds S4

3. Additional experiments $\quad S 10$

4. References $\quad S 16$

5. Characterization of New Compounds $\quad$ S17 


\section{General Experimental}

All commercially available reagents and solvents were purchased from Sigma-Aldrich, Fluorochem and Alfa Aesar. They were all used as received. Flash chromatography was carried out using Merck silica gel 60 (pore size $60 \AA, 270-400 \mathrm{Mesh}) .{ }^{1} \mathrm{H}$ and ${ }^{13} \mathrm{C}$ NMR spectra were recorded from solutions in deuterated solvents on 300 Bruker spectrometers or 400 Jeol with tetramethylsilane as internal standard. Low resolution mass spectra of pure compounds were recorded using Agilent Technologies ESI-MS Spectrometer instrument and a Thermofisher Finnigan TRACE DSQ GCMS instrument equipped with Direct Exposure Probe (DEP). Samples for the ESI-MS experiment were dissolved in a mixture of THF/MeOH 1:1, while samples for the DEP-MS were dissolved in a good solvent (eg. DCM) and settled on the probe filament. High resolution mass spectra were recorded using a Bruker Autoflex MALDI-TOF in reflectron mode with trans-2-[3-(4-tertButylphenyl)-2-methyl-2-propenylidene]malononitrile (DCTB) as a matrix. The UV-Vis spectroscopic studies were recorded using JASCO V-550 spectrophotometer. The PL and PLE spectra were recorded using Perkin Elmer LS55 luminescence spectrophotometer and Horiba Fluorolog. Cyclic voltammetry experiments were carried out using a Biologic SP-150 potentiostat with a polished glassy carbon working electrode, platinum counter electrode, silver pseudoreference electrode, and tetrabutylammonium hexofluorophosphate (recrystallized three times from $\mathrm{EtOH}$ ) as a supporting electrolyte. Sample concentrations were between 0.2 and $1.0 \mathrm{mM}$. All electrochemical measurements were reference to the $\mathrm{Fc} / \mathrm{Fc}^{+}$redox couple. Band gaps were estimated using the onset of the initial oxidation and reduction events, and $\mathrm{E}_{\mathrm{HOMO}}$ and $\mathrm{E}_{\mathrm{LUMO}}$ were estimated given an $\mathrm{E}_{\text {номо }}$ of $4.80 \mathrm{eV}$ for ferrocene. ${ }^{1}$ Theoretical calculations were carried out with the Spartan '18 (1.4.4, Wave function Inc, Irvine CA) software packages on a computer operating with Windows 10 OS. Geometry optimizations and energy calculations was performed with density functional theory (DFT) calculations using the $\omega \mathrm{B} 97 \mathrm{X}-\mathrm{D}$ functional with the $6-31 \mathrm{G}^{*}$ basis set in the gas phase. Solubilizing alkyl groups were replaced with methyl groups for simplicity.

\section{Synthesis of known compounds}

Scheme S1. Synthesis of aldehyde $3 g$.

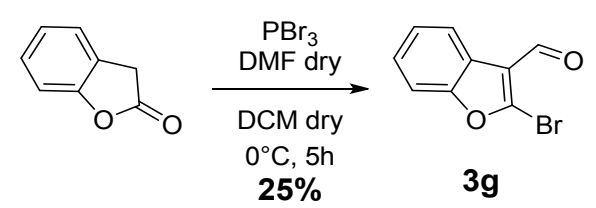

Compounds 3g was synthesized in one step adapting the Vilsmeier-Hack protocol reported in literature for compound $\mathbf{3 h} .^{2}$

2-bromobenzofuran-3-carbaldehyde (3g). A dried double neck flask purged with argon and sealed with a septum cap was charged with dry DCM $(1 \mathrm{~mL})$ and dry DMF $(1 \mathrm{~mL})$, cooled to $0{ }^{\circ} \mathrm{C}$ with an ice-water bath and $\mathrm{PBr}_{3}(469 \mu \mathrm{L}, 5 \mathrm{mmol}, 2.5 \mathrm{eq})$ was added dropwise. The solution was stirred for $1 \mathrm{~h}$ at $0{ }^{\circ} \mathrm{C}$, then benzofuran-2(3H)-one (268 mg, $2 \mathrm{mmol}, 1.0 \mathrm{eq})$ was dissolved in dry DCM (3 mL) and added via syringe. 
The reaction was refluxed for $12 \mathrm{~h}$ with an oil bath. After TLC monitoring, the solvent was removed under reduced pressure yielding an orange-brown lumpy solid. Purification by column chromatography $\left(\mathrm{SiO}_{2}, 8: 2\right.$ diethyl ether : $n$-hexane) yielded pure $\mathbf{3 g}$ as a pale yellow solid (220 mg, 46\%). ${ }^{1} \mathrm{H} \mathrm{NMR}\left(200 \mathrm{MHz}, \mathrm{CDCl}_{3}\right)$ $\delta=10.05(\mathrm{~s}, 1 \mathrm{H}), 8.13(\mathrm{dd}, J=5.6,3.1 \mathrm{~Hz}, 1 \mathrm{H}), 7.43-7.50(\mathrm{~m}, 1 \mathrm{H}), 7.33-7.40(\mathrm{~m}, 2 \mathrm{H})$.

Scheme S2. Synthesis of aldehyde $3 \mathrm{~h}$

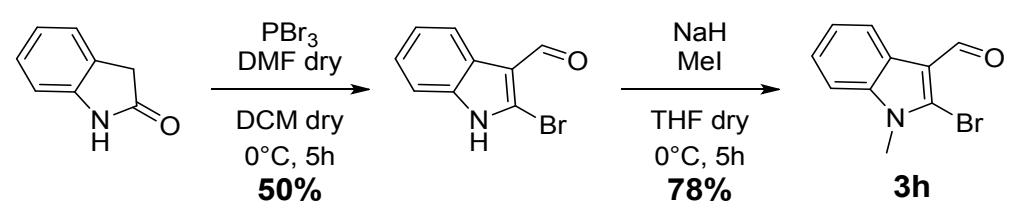

Compounds $\mathbf{3 h}$ was synthetized adapting a Vilsmeier-Hack protocol reported in the literature. ${ }^{2}$

2-bromo-1H-indole-3-carbaldehyde. A dried double neck flask purged with argon and sealed with a septum cap was charged with dry DCM $(6.0 \mathrm{~mL})$ and dry DMF $(1.8 \mathrm{~mL})$, cooled to $0{ }^{\circ} \mathrm{C}$ with an ice-water bath and $\mathrm{PBr}_{3}(5.8 \mathrm{~g}, 19 \mathrm{mmol}, 2.5 \mathrm{eq})$ was added dropwise. The solution was stirred for 1 hour at $0{ }^{\circ} \mathrm{C}$, then indolin-2one $(1.0 \mathrm{~g}, 7.5 \mathrm{mmol}, 1.0 \mathrm{eq})$ in dry DCM $(10 \mathrm{~mL})$ was added via syringe. The reaction was refluxed for $5 \mathrm{~h}$ with an oil bath. During this time the reaction mixture showed an initial orange-white double layer changing into a limpid copper color. The color kept on changing into dark brown till a final dark blue. When a TLC check showed the reaction ending, the solvent was removed under reduced pressure yielded an orange-brown lumpy solid. Purification by column chromatography $\left(\mathrm{SiO}_{2}, 8: 2\right.$ diethyl ether : $n$-hexane) yielded the pure product (507.8 mg, 50\%). ${ }^{1} \mathrm{H}$ NMR (200 MHz, DMSO- $\left.d_{6}\right) \delta=13.03(\mathrm{~s}, 1 \mathrm{H}), 9.89$ (s, 1H), 8.07 (dd, $J=5.6$, $3.1 \mathrm{~Hz}, 1 \mathrm{H}), 7.43(\mathrm{dd}, J=5.9,2.8 \mathrm{~Hz}, 1 \mathrm{H}), 7.35-7.11(\mathrm{~m}, 2 \mathrm{H}) .{ }^{13} \mathrm{C} \mathrm{NMR}\left(75 \mathrm{MHz}, \mathrm{CDCl}_{3}\right) \delta=184.5,136.4$, $124.8,123.8,123.3,122.7,119.7,114.3,111.7$

2-bromo-1-methyl-1H-indole-3-carbaldehyde (3h). A dried double neck flask purged with argon was charged with 2-bromo-1H-indole-3-carbaldehyde (300 mg, $1.34 \mathrm{mmol}, 1.0 \mathrm{eq})$, dry THF (14 mL), cooled to $0^{\circ} \mathrm{C}$ with an ice-water bath and $\mathrm{NaH}(96.4 \mathrm{mg}, 4.0 \mathrm{mmol}, 3.0 \mathrm{eq})$ was added in one portion. A color change from red to green was observed. The reaction mixture was kept under stirring for $30 \mathrm{~min}$, then iodomethane ( $570 \mathrm{mg}, 4.0 \mathrm{mmol}, 3.0 \mathrm{eq}$ ) was added and the color changed to orange. The reaction was warmed to room temperature and kept for $2 \mathrm{~h}$. The reaction was quenched with water and the solvents were removed under reduced pressure. The reaction mixture was purified by flash chromatography $\left(\mathrm{SiO}_{2} ; n\right.$-hexane : AcOEt $\left.8: 2\right)$ affording a yellow solid (223 mg, 78\%). ${ }^{1} \mathrm{H}$ NMR (200 MHz, $\left.\mathrm{CDCl}_{3}\right) \delta=10.05$ (s, 1H), 8.44-8.16 (m, 1H), $7.35(\mathrm{~s}, 3 \mathrm{H}), 3.86(\mathrm{~s}, 3 \mathrm{H})$.

Scheme S3. Synthesis of aldehyde $3 \mathrm{f}$.

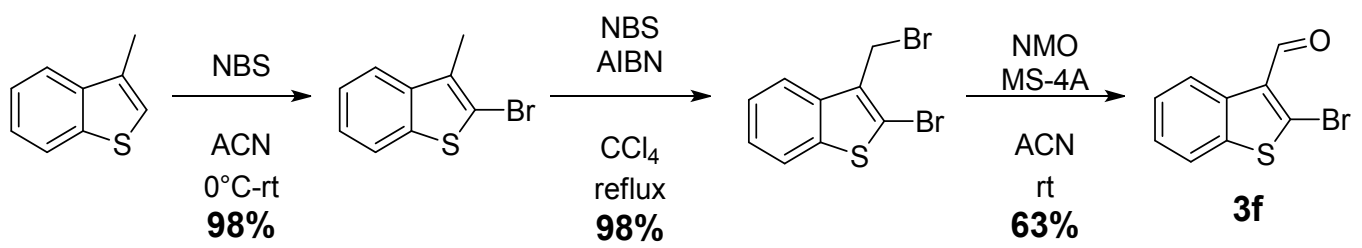

Compounds $\mathbf{3 f}$ was synthetized adapting protocols reported in literature. $3,4,5$ 
2-bromo-3-methylbenzo[b]thiophene. A $10 \mathrm{~mL}$ round-bottom flask was equipped with a stir bar and cooled to $0{ }^{\circ} \mathrm{C}$ with an ice-water bath. Sequentially, 3-methylbenzothiophene $(1,31 \mathrm{~mL}, 10 \mathrm{mmol}, 1 \mathrm{eq}), \mathrm{MeCN}(12$ $\left.\mathrm{mL}, 0.85 \mathrm{~mol} \cdot \mathrm{L}^{-1}\right)$, and NBS $(1.78 \mathrm{~g}, 10 \mathrm{mmol}, 1 \mathrm{eq})$ were added. The ice-water bath was removed after $5 \mathrm{~min}$ and the solution was stirred at $\mathrm{rt}$ for $1 \mathrm{~h}$. The reaction was quenched with water $(50 \mathrm{~mL})$ and $\mathrm{MeCN}$ was removed. The residue was extracted with DCM $(3 \times 50 \mathrm{~mL})$. The combined organic layers were dried over $\mathrm{Na}_{2} \mathrm{SO}_{4}$, filtered, and concentrated in vacuo. The resulting oil was purified via filtration on a short silica gel path using hexanes as the eluent, to give $2.22 \mathrm{~g}$ of a pale yellow oil $(98 \%) .{ }^{1} \mathrm{H} \mathrm{NMR}\left(200 \mathrm{MHz}, \mathrm{CDCl}_{3}\right) \delta=$ $7.73(\mathrm{dd}, J=6.1,2.7 \mathrm{~Hz}, 1 \mathrm{H}), 7.65(\mathrm{dd}, J=6.1,2.7 \mathrm{~Hz}, 1 \mathrm{H}), 7.41-7.32(\mathrm{~m}, 2 \mathrm{H}), 2.40(\mathrm{~s}, 2 \mathrm{H})$.

2-Bromo-3-(bromomethyl)methylbenzo $[b]$ thiophene. To a solution of 2-bromo-3-methylbenzo $[b]$ thiophene $(2.27 \mathrm{~g}, 10 \mathrm{mmol}, 1 \mathrm{eq})$ in dry $\mathrm{CCl}_{4}\left(10 \mathrm{~mL}, 1 \mathrm{~mol} \cdot \mathrm{L}^{-1}\right)$ were added AIBN (49 $\mathrm{mg}, 0.03 \mathrm{mmol}, 0.03$ eq) and finely powdered NBS (1.78 g, $10 \mathrm{mmol}, 1 \mathrm{eq})$. The reaction mixture was heated at reflux in an oil bath for $30 \mathrm{~min}$ and cooled to room temperature. The floating succinimide was filtered off and washed with carbon tetrachloride $(15 \mathrm{~mL})$. The combined filtrate was concentrated in vacuo to afford $2.99 \mathrm{~g}$ of 2-bromo-3bromomethythiophene $(98 \%)$ as a white solid. ${ }^{1} \mathrm{H}$ NMR $\left(200 \mathrm{MHz}, \mathrm{CDCl}_{3}\right) \delta=7.80(\mathrm{~d}, J=7.2 \mathrm{~Hz}, 1 \mathrm{H}), 7.74$ (d, $J=7.2 \mathrm{~Hz}, 1 \mathrm{H}), 7.41(\mathrm{~m}, 2 \mathrm{H}), 4.71(\mathrm{~s}, 2 \mathrm{H})$.

2-bromobenzo[b]thiophene-3-carbaldehyde (3f). To a stirring mixture of $N$-methylmorpholine- $N$-oxide (1.76 g, $15 \mathrm{mmol}, 3 \mathrm{eq})$ and $4 \AA$ molecular sieves $(11 \mathrm{~g})$ in $\mathrm{MeCN}\left(50 \mathrm{~mL}, 0.1 \mathrm{~mol} \cdot \mathrm{L}^{-1}\right)$ at $0^{\circ} \mathrm{C}$ in an ice-water bath under $\mathrm{N}_{2}$ was added dropwise neat 2-bromo-3-(bromomethyl)thiophene (1.53 g, 5 mmol, 1 eq) over 5 min via syringe. After $4 \mathrm{~h}$, the reaction mixture was filtered directly through a short pad of $\mathrm{SiO}_{2}$, eluting with $600 \mathrm{~mL}$ of EtOAc. The organic solvent was removed under reduced pressure to give $0.763 \mathrm{~g}$ of aldehyde $\mathbf{3 f}$ as a yellow oil $(63 \%)$, which became an off-white solid after storage at $5^{\circ} \mathrm{C} .{ }^{1} \mathrm{H}$ NMR $\left(400 \mathrm{MHz}, \mathrm{CDCl}_{3}\right) \delta=$ $9.95(\mathrm{~s}, 1 \mathrm{H}), 7.36$ (d, 1H, $J=6.0 \mathrm{~Hz}), 7.29$ (d, 1H, $J=6.0 \mathrm{~Hz})$.

\section{Synthesis of new compounds}

General procedure for the DHA-cross aldol condensation. Selected example for compound 8. 3-Bromo-2thiophenecarboxaldehyde $3 \mathbf{c}(191 \mathrm{mg}, 1 \mathrm{mmol}, 1 \mathrm{eq})$ was added to a solution of 3-thiopheneacetic acid (142 $\mathrm{mg}, 1 \mathrm{mmol}, 1 \mathrm{eq}), \mathrm{Pd}(\mathrm{OAc})_{2}$ (11 mg, $\left.0.05 \mathrm{mmol}, 0.05 \mathrm{eq}\right), \mathrm{PPh}_{3}(26 \mathrm{mg}, 0.02 \mathrm{mmol}, 0.1 \mathrm{eq}), \mathrm{K}_{2} \mathrm{CO}_{3}(414 \mathrm{mg}$, $3 \mathrm{mmol}, 3 \mathrm{eq})$ in dry DMF $\left(5 \mathrm{~mL}, 0.2 \mathrm{~mol} \cdot \mathrm{L}^{-1}\right)$ under inert atmosphere and then stirred at $110{ }^{\circ} \mathrm{C}$ with an oil bath for $12 \mathrm{~h}$. The reaction suspension was cooled to $\mathrm{rt}$ and iodomethane ( $160 \mu \mathrm{L}, 3 \mathrm{mmol}, 3 \mathrm{eq})$ was added in one portion. The reaction mixture was kept under stirring for a further $4 \mathrm{~h}$. After removal of solvent under reduced pressure, the crude of reaction was purified by short flash chromatography $\left(\mathrm{SiO}_{2} ; n\right.$-hexanes : AcOEt 95:5).

Methyl benzo[1,2-b:3,4-b'] dithiophene-4-carboxylate (8). White solid (206 mg, 83\%). ${ }^{1} \mathrm{H}$ NMR (300 MHz, $\left.\mathrm{CDCl}_{3}\right) \delta=8.54(\mathrm{t}, J=0.9 \mathrm{~Hz}, 1 \mathrm{H}), 8.30(\mathrm{dd}, J=5.5,0.9 \mathrm{~Hz}, 1 \mathrm{H}), 7.65(\mathrm{dd}, J=5.4,1.0 \mathrm{~Hz}, 1 \mathrm{H}), 7.50(\mathrm{dd}, J$ $=5.5,0.9 \mathrm{~Hz}, 1 \mathrm{H}), 7.47(\mathrm{dd}, J=5.4,1.0 \mathrm{~Hz}, 1 \mathrm{H}), 3.99(\mathrm{~s}, 3 \mathrm{H}) .{ }^{13} \mathrm{C} \mathrm{NMR}\left(75 \mathrm{MHz}, \mathrm{CDCl}_{3}\right) \delta=167.2,137.9$, 
136.0, 125.9, 125.6, 125.5, 125.1, 125.0, 121.7, 52.0. DEP-MS: $m / z$ 248. Anal. Calcd. For $\mathrm{C}_{12} \mathrm{H}_{8} \mathrm{O}_{2} \mathrm{~S}_{2}: \mathrm{C}_{\text {, 58.0; }}$; $\mathrm{H}, 3.2$. Found: $\mathrm{C}, 58.0 ; \mathrm{H}, 3.2$.

benzo[1,2-b:6,5-b']dithiophene-4-carboxylic acid (9). Grey solid (206 mg, 89\%). ${ }^{1} \mathrm{H}$ NMR (300 MHz, DMSO$\left.d_{6}\right) \delta=13.16(\mathrm{~s}, 1 \mathrm{H}), 8.71(\mathrm{~s}, 1 \mathrm{H}), 8.29(\mathrm{~d}, J=5.5 \mathrm{~Hz}, 1 \mathrm{H}), 8.18(\mathrm{~d}, J=5.5 \mathrm{~Hz}, 1 \mathrm{H}), 7.91(\mathrm{~d}, J=5.5 \mathrm{~Hz}, 1 \mathrm{H})$, $7.79(\mathrm{~d}, J=5.4 \mathrm{~Hz}, 1 \mathrm{H}) .{ }^{13} \mathrm{C}$ NMR $\left(75 \mathrm{MHz}, \mathrm{DMSO}-d_{6}\right) \delta=167.53,136.38,135.42,134.88,134.75,133.22$, 126.93, 125.16, 123.33, 121.81, 121.65. ESI-MS: $m / z 233$ [M-1]-, 467 [2M-1]-. Anal. Calcd. for $\mathrm{C}_{11} \mathrm{H}_{6} \mathrm{O}_{2} \mathrm{~S}_{2}$ : C, 56.6; H, 2.1. Found: C, 56.6; H, 2.2.

methyl thieno[2,3-e]benzofuran-4-carboxylate (10). Beige solid (177 mg, 76\%). $\left.{ }^{1} \mathrm{H} \mathrm{NMR} \mathrm{(300} \mathrm{MHz,} \mathrm{CDCl}_{3}\right)$ $\delta=13.15(\mathrm{~s}, 1 \mathrm{H}), 8.71(\mathrm{~s}, 1 \mathrm{H}), 8.30(\mathrm{~d}, \mathrm{~J}=5.5 \mathrm{~Hz}, 1 \mathrm{H}), 8.19(\mathrm{~d}, \mathrm{~J}=5.4 \mathrm{~Hz}, 1 \mathrm{H}), 7.91(\mathrm{~d}, \mathrm{~J}=5.5 \mathrm{~Hz}, 1 \mathrm{H}), 7.80$ $(\mathrm{d}, \mathrm{J}=5.4 \mathrm{~Hz}, 1 \mathrm{H}) .{ }^{13} \mathrm{C}$ NMR $\left(75 \mathrm{MHz}, \mathrm{CDCl}_{3}\right) \delta=167.5,136.4,135.4,134.9,134.8,133.2,126.9,125.17$, 123.3, 121.8, 121.7. DEP-MS: $m / z$ 232. Anal. Calcd. for $\mathrm{C}_{12} \mathrm{H}_{8} \mathrm{O}_{3} \mathrm{~S}: \mathrm{C}, 62.06 ; \mathrm{H}, 3.47$. Found: C, 62.0; H, 3.4 . thieno[2,3-e]benzofuran-4-carboxylic acid (11). Brown solid (168 mg, 77\%). ${ }^{1} \mathrm{H}$ NMR (300 MHz, DMSO- $d_{6}$ ) $\delta=13.15(\mathrm{~s}, 1 \mathrm{H}), 8.52-8.14(\mathrm{~m}, 1 \mathrm{H}), 7.89(\mathrm{~d}, J=5.6 \mathrm{~Hz}, 1 \mathrm{H}), 7.37(\mathrm{~s}, 1 \mathrm{H}) .{ }^{13} \mathrm{C}$ NMR $\left(75 \mathrm{MHz}, \mathrm{DMSO}-d_{6}\right)$ $\delta=167.5,136.4,135.4,134.9,134.8,133.2,126.9,125.17,123.3,121.8,121.7$. ESI-MS: $m / z: 217$ [M-1].. Anal. Calcd. for $\mathrm{C}_{11} \mathrm{H}_{6} \mathrm{O}_{3} \mathrm{~S}$ : C, 56.6; H, 2.2. Found: C, 56.6; H, 2.2.

methyl 1H-benzo[b] thiophene[4,5-d] thiophene-4-carboxylate (12).Pale yellow solid (226 mg, 76\%). ${ }^{1} \mathrm{H}$ NMR $\left(300 \mathrm{MHz}, \mathrm{CDCl}_{3}\right) \delta=8.64(\mathrm{~s}, 1 \mathrm{H}), 8.55-8.37(\mathrm{~m}, 2 \mathrm{H}), 7.98(\mathrm{dd}, J=6.6,1.7 \mathrm{~Hz}, 1 \mathrm{H}), 7.76-7.44(\mathrm{~m}, 3 \mathrm{H})$, $4.05(\mathrm{~s}, 3 \mathrm{H}) .{ }^{13} \mathrm{C} \mathrm{NMR}\left(75 \mathrm{MHz}, \mathrm{CDCl}_{3}\right) \delta=166.7,141.2,136.5,135.2,135.0,134.0,132.5,127.1,125.8$, 124.9, 124.7, 124.4, 122.9, 122.8, 122.7, 52.2. DEP-MS: $m / z$ 298. Anal. Calcd. for $\mathrm{C}_{16} \mathrm{H}_{10} \mathrm{O}_{2} \mathrm{~S}_{2}$ : C, 64.4; $\mathrm{H}$, 3.4. Found: C, 64.4; H, 3.3.

methyl 1H-benzo[b] thiophene[5,4-d] thiophene-4-carboxylate (13). Pale yellow solid (161 mg, 54\%). ${ }^{1} \mathrm{H}$ NMR $\left(300 \mathrm{MHz}, \mathrm{CDCl}_{3}\right) \delta=8.88(\mathrm{~s}, 1 \mathrm{H}), 8.37(\mathrm{~d}, J=5.5 \mathrm{~Hz}, 1 \mathrm{H}), 8.30-8.19(\mathrm{~m}, 1 \mathrm{H}), 7.95-7.86(\mathrm{~m}, 1 \mathrm{H}), 7.62$ $(\mathrm{d}, J=5.5 \mathrm{~Hz}, 1 \mathrm{H}), 7.59-7.43(\mathrm{~m}, 2 \mathrm{H}), 4.06(\mathrm{~s}, 3 \mathrm{H}) .{ }^{13} \mathrm{C} \mathrm{NMR}\left(75 \mathrm{MHz}, \mathrm{CDCl}_{3}\right) \delta=166.7,141.2,136.5$, 135.2, 135.0, 134.0, 132.5, 127.1, 125.8, 124.9, 124.7, 124.4, 122.9, 122.8, 122.7, 52.2. DEP-MS: $\mathrm{m} / z 298$. Anal. Calcd. for $\mathrm{C}_{16} \mathrm{H}_{10} \mathrm{O}_{2} \mathrm{~S}_{2}$ : C, $64.40 \mathrm{H}$, 3.4. Found: C, 64.4; H, 3.3.

Methyl benzo[b] thieno[3,2-g]benzofuran-4-carboxylate (14). Pale yellow solid (121 mg, 43\% yield). ${ }^{1} \mathrm{H}$ NMR $\left(200 \mathrm{MHz}, \mathrm{CDCl}_{3}\right) \delta=8.88(\mathrm{~s}, 1 \mathrm{H}), 8.37(\mathrm{~d}, J=5.5 \mathrm{~Hz}, 1 \mathrm{H}), 8.30-8.19(\mathrm{~m}, 1 \mathrm{H}), 7.95-7.86(\mathrm{~m}, 1 \mathrm{H}), 7.62$ $(\mathrm{d}, J=5.5 \mathrm{~Hz}, 1 \mathrm{H}), 7.59-7.43(\mathrm{~m}, 2 \mathrm{H}), 4.06(\mathrm{~s}, 3 \mathrm{H}) .{ }^{13} \mathrm{C} \mathrm{NMR}\left(75 \mathrm{MHz}, \mathrm{CDCl}_{3}\right) \delta=166.7,141.2,136.5$, 135.2, 135.0, 134.0, 132.5, 127.1, 125.8, 124.9, 124.7, 124.4, 122.9, 122.8, 122.7, 52.2. ESI-MS: $m / z 283$ $[\mathrm{M}+1]^{+}$. Anal. Calcd. for $\mathrm{C}_{16} \mathrm{H}_{10} \mathrm{O}_{3} \mathrm{~S}: \mathrm{C}, 68.1 ; \mathrm{H}, 3.6$. Found: $\mathrm{C}, 68.0 ; \mathrm{H}, 3.5$.

methyl 10-methyl-10H-thieno[2,3-a]carbazole-4-carboxylate (15). Yellow solid (38 mg, 13\%) ${ }^{1} \mathrm{H}$ NMR (300 $\left.\mathrm{MHz}, \mathrm{CDCl}_{3}\right) \delta=8.12(\mathrm{dd}, J=13.8,8.0 \mathrm{~Hz}, 2 \mathrm{H}), 7.68(\mathrm{~d}, J=8.3 \mathrm{~Hz}, 1 \mathrm{H}), 7.55(\mathrm{~d}, J=5.3 \mathrm{~Hz}, 1 \mathrm{H}), 7.51-$ $7.43(\mathrm{~m}, 3 \mathrm{H}), 7.31(\mathrm{~s}, 1 \mathrm{H}), 4.22(\mathrm{~s}, 3 \mathrm{H}) .{ }^{13} \mathrm{C} \mathrm{NMR}\left(75 \mathrm{MHz}, \mathrm{CDCl}_{3}\right) \delta=167.6,140.8,138.6,138.4,125.8$, 125.5, 125.4, 123.3, 122.8, 122.2, 120.2, 120.0, 117.5, 115.9, 108.9. ESI-MS: $m / z 296[\mathrm{M}+1]^{+}$. Anal. Calcd. for $\mathrm{C}_{17} \mathrm{H}_{13} \mathrm{NO}_{2} \mathrm{~S}$ : C, 69.1; H, 4.4. Found: C, 69.1; H, 4.4. 
10-methyl-10H-thieno[2,3-a]carbazole (16). Yellow solid (69 mg, 29\%). ${ }^{1} \mathrm{H}$ NMR (300 $\left.\mathrm{MHz}, \mathrm{CDCl}_{3}\right) \delta=$ 8.79 (s, 1H), $8.53(\mathrm{~d}, J=5.5 \mathrm{~Hz}, 1 \mathrm{H}), 8.12(\mathrm{~d}, J=7.7 \mathrm{~Hz}, 1 \mathrm{H}), 7.59$ (d, $J=5.5 \mathrm{~Hz}, 1 \mathrm{H}), 7.53-7.20(\mathrm{~m}, 3 \mathrm{H})$, $4.12(\mathrm{~s}, 3 \mathrm{H}), 4.04(\mathrm{~s}, 3 \mathrm{H}) \cdot{ }^{13} \mathrm{C} \mathrm{NMR}\left(75 \mathrm{MHz}, \mathrm{CDCl}_{3}\right) \delta=167.6,140.8,138.6,138.4,125.8,125.5,125.4$, 123.3, 122.8, 122.2, 120.2, 120.0, 117.5, 115.9, 108.9, 51.7, 31.1. DEP-MS: $m / z$ 237. Anal. Calcd. for $\mathrm{C}_{15} \mathrm{H}_{11} \mathrm{NS}: \mathrm{C}, 75.9 ; \mathrm{H}, 4.7$. Found: C, 75.9; H, 4.6.

Methyl thieno[3,2-h]quinoline-4-carboxylate (17).Pale yellow solid (56 mg, 23\% yield). ${ }^{1} \mathrm{H}$ NMR (300 MHz, $\left.\mathrm{CDCl}_{3}\right) \delta=8.99(\mathrm{dd}, J=4.3,1.7 \mathrm{~Hz}, 1 \mathrm{H}), 8.52(\mathrm{~s}, 1 \mathrm{H}), 8.35-8.28(\mathrm{~m}, 2 \mathrm{H}), 7.76(\mathrm{~d}, J=5.5 \mathrm{~Hz}, 1 \mathrm{H}), 7.50$ $(\mathrm{dd}, J=8.2,4.4 \mathrm{~Hz}, 1 \mathrm{H}), 4.04(\mathrm{~s}, 3 \mathrm{H}) .{ }^{13} \mathrm{C} \mathrm{NMR}\left(75 \mathrm{MHz}, \mathrm{CDCl}_{3}\right) \delta=166.5,151.7,146.0,140.2,137.8$, 137.8, 129.6, 129.1, 125.7, 124.1, 124.0, 121.5, 52.3. ESI-MS: $m / z 244[\mathrm{M}+1]^{+}$. Anal. Calcd. for $\mathrm{C}_{13} \mathrm{H}_{9} \mathrm{NO}_{2} \mathrm{~S}$ : C, 64.2; H, 3.7. Found: C, 64.1; H, 3.7.

\section{General procedure for the DHA-cross aldol condensation and alkylation with 9-} (bromomethyl)nonadecane. Selected example for compound 18. 3-bromobenzo[b] thiophene-2-carbaldehyde $3 \mathbf{e}(2.41 \mathrm{~g}, 10 \mathrm{mmol}, 1 \mathrm{eq})$ was added to a solution of 3 -thiopheneacetic acid (1.42 g, $10 \mathrm{mmol}, 1 \mathrm{eq}), \mathrm{Pd}(\mathrm{OAc})_{2}$ (224 mg, $1 \mathrm{mmol}, 0.1 \mathrm{eq}), \mathrm{PPh}_{3}(524 \mathrm{mg}, 2 \mathrm{mmol}, 0.2 \mathrm{eq}), \mathrm{K}_{2} \mathrm{CO}_{3}(2.76 \mathrm{~g}, 20 \mathrm{mmol}, 2 \mathrm{eq})$ in dry DMF (20 $\left.\mathrm{mL}, 0.2 \mathrm{~mol} \cdot \mathrm{L}^{-1}\right)$ under inert atmosphere and then stirred at $110{ }^{\circ} \mathrm{C}$ in an oil bath for $24 \mathrm{~h}$. The reaction suspension was cooled to $60^{\circ} \mathrm{C}$ and 9-(bromomethyl)nonadecane (3.62 g, $10 \mathrm{mmol}, 1 \mathrm{eq}$ ) was added in one portion. The reaction mixture was kept under stirring for a further $12 \mathrm{~h}$. After removal of solvent under reduced pressure, the crude of reaction was purified by flash chromatography $\left(\mathrm{SiO}_{2} ; \mathrm{d}=5 \mathrm{~cm}, \mathrm{~h}=35 \mathrm{~cm}\right.$; eluent: $n$ hexanes : AcOEt $98: 2$ ). Pure product was obtained as a pale yellow oil (2.88 g, 51\%). TLC 7:3 $n$-hexanes : AcOEt, $R_{\mathrm{f}}=0.49$.

2-octyldodecyl benzo[b]thieno[2,3-e]benzothiophene-4-carboxylate (18). Pale Yellow oil (2.88 g, 51\%). ${ }^{1} \mathrm{H}$ NMR $\left(200 \mathrm{MHz}, \mathrm{CDCl}_{3}\right) \delta=8.62(\mathrm{~s}, 1 \mathrm{H}), 8.56-8.42(\mathrm{~m}, 2 \mathrm{H}), 7.98(\mathrm{dd}, J=6.9,2.0 \mathrm{~Hz}, 1 \mathrm{H}), 7.73-7.45(\mathrm{~m}$, $3 \mathrm{H}), 4.37(\mathrm{~d}, J=5.7 \mathrm{~Hz}, 2 \mathrm{H}), 1.96-1.75(\mathrm{~m}, 1 \mathrm{H}), 1.54-1.13(\mathrm{~m}, 32 \mathrm{H}), 0.96-0.77(\mathrm{~m}, 6 \mathrm{H}) .{ }^{13} \mathrm{C}$ NMR $(75$ $\left.\mathrm{MHz}, \mathrm{CDCl}_{3}\right) \delta=168.5,138.2,137.4,137.1,135.4,134.3,131.3,126.5,125.5,124.7,122.6,121.9,121.5$, 121.3, 77.2, 76.7, 76.3, 67.8, 37.3, 31.6, 31.4, 29.8, 29.4, 29.4, 29.3, 29.1, 26.6, 22.4, 13.8. DEP-MS: $m / z 565$. Anal. Calcd. for $\mathrm{C}_{35} \mathrm{H}_{48} \mathrm{O}_{2} \mathrm{~S}_{2}$ : C, 74.4; H, 8.6. Found: C, 74.2; H, 8.9.

2-octyldodecyl benzo[b]thieno[3,2-g]benzothiophene-4-carboxylate (20). Pale Yellow oil (2.60 g, 46\%). ${ }^{1} \mathrm{H}$ NMR $\left(300 \mathrm{MHz}, \mathrm{CDCl}_{3}\right) \delta=8.78(\mathrm{~s}, 1 \mathrm{H}), 8.35(\mathrm{~d}, J=5.5 \mathrm{~Hz}, 1 \mathrm{H}), 8.19-8.08(\mathrm{~m}, 1 \mathrm{H}), 7.90-7.75(\mathrm{~m}, 1 \mathrm{H})$, 7.57 (d, $J=5.4 \mathrm{~Hz}, 1 \mathrm{H}), 7.46$ (hept, $J=5.4 \mathrm{~Hz}, 2 \mathrm{H}), 4.39$ (d, $J=5.7 \mathrm{~Hz}, 2 \mathrm{H}), 1.93$ (t, $J=5.8 \mathrm{~Hz}, 1 \mathrm{H}), 1.59-$ $1.18(\mathrm{~m}, 32 \mathrm{H}), 0.89$ (t, $J=6.5 \mathrm{~Hz}, 6 \mathrm{H}) .{ }^{13} \mathrm{C} \mathrm{NMR}\left(75 \mathrm{MHz}, \mathrm{CDCl}_{3}\right) \delta=166.5,138.2,137.6,137.2,135.4$, 134.4, 131.3, 126.5, 125.5, 124.7, 122.6, 121.9, 121.5, 121.3, 77.2, 76.7, 76.3, 67.8, 37.3, 31.6, 31.4, 29.8, 29.4, 29.4, 29.3, 29.1, 26.6, 22.4, 13.8. DEP-MS: $m / z$ 565. Anal. Calcd. for $\mathrm{C}_{35} \mathrm{H}_{48} \mathrm{O}_{2} \mathrm{~S}_{2}: \mathrm{C}, 74.4 ; \mathrm{H}, 8.6$. Found: C, 74.2; H, 8.8. 
General procedure for stannylation. Selected example: compound 19. A solution of compound $\mathbf{1 8}$ ( $1 \mathrm{~g}, 1.77$ mmol, 1eq) in dry THF $(35 \mathrm{~mL})$ under argon atmosphere was cooled to $-78^{\circ} \mathrm{C}$ in a dry ice-acetone bath. After $15 \mathrm{~min}$ a $1 \mathrm{M}$ solution of LDA in Hexane $(2.66 \mathrm{~mL} ; 2.66 \mathrm{mmol}, 1.5 \mathrm{eq})$ was added dropwise and kept for $2 \mathrm{~h}$ at same temperature, than $\mathrm{Bu}_{4} \mathrm{SnCl}(960 \mu \mathrm{L}, 3.54 \mathrm{mmol}, 2 \mathrm{eq})$ was added in one portion. The reaction mixture after $30 \mathrm{~min}$ at $-78^{\circ} \mathrm{C}$ was warmed to room temperature and kept at same temperature overnight. The reaction mixture was quenched with water, the solvent removed under reduced pressure, extracted with $\mathrm{Et}_{2} \mathrm{O}$ ( 3 times) and collected organic phases was dried over $\mathrm{Na}_{2} \mathrm{SO}_{4}$. After removal of the solvent under reduced pressure, crude of reaction was purified by flash chromathography on silica gel (eluent: $n$-hexanes) previously conditioned with a mixture of $9: 1 n$-hexanes: triethylamine (TEA) and washed with n-Hexane affording pure compound as colorless oil $(1.10 \mathrm{~g}, 73 \%)$.

2-octyldodecyl 2-(tributylstannyl)benzo[b]thieno[2,3-e]benzothiophene-4-carboxylate (19). Pale yellow oil (1.10 g, 73\%). ${ }^{1} \mathrm{H}$ NMR (400 MHz, $\left.\mathrm{CDCl}_{3}\right) \delta=8.68-8.44(\mathrm{~m}, 3 \mathrm{H}), 7.99(\mathrm{~d}, J=7.5 \mathrm{~Hz}, 1 \mathrm{H}), 7.72-7.52$ (m, $2 \mathrm{H}), 4.38(\mathrm{~d}, J=5.4 \mathrm{~Hz}, 2 \mathrm{H}), 1.98-1.77(\mathrm{~m}, 1 \mathrm{H}), 1.76-1.14(\mathrm{~m}, 50 \mathrm{H}), 1.01-0.78(\mathrm{~m}, 15 \mathrm{H}) .{ }^{13} \mathrm{C}$ NMR $(75$ $\left.\mathrm{MHz}, \mathrm{CDCl}_{3}\right) \delta=168.1,141.5,140.1,139.9,139.0,138.3,136.9,133.9,130.5,126.3,125.2,123.2,122.1$, $121.8,121.6,37.3,32.1,31.8,30.3,29.6,29.5,29.3,29.2,29.1,28.8,27.4,27.1,22.5,14.1,13.6,11.1$.

2-octyldodecyl 2-(tributylstannyl)benzo[b]thieno[3,2-g]benzothiophene-4-carboxylate (21). Pale yellow oil (1.19 g, 79\%). ${ }^{1} \mathrm{H}$ NMR (300 MHz, $\left.\mathrm{CDCl}_{3}\right) \delta=8.86(\mathrm{~s}, 1 \mathrm{H}), 8.43(\mathrm{~s}, 1 \mathrm{H}), 8.24(\mathrm{dd}, J=7.6,1.6 \mathrm{~Hz}, 1 \mathrm{H}), 7.90$ $(\mathrm{d}, J=1.8 \mathrm{~Hz}, 1 \mathrm{H}), 7.57-7.39(\mathrm{~m}, 2 \mathrm{H}), 4.40(\mathrm{~d}, J=5.7 \mathrm{~Hz}, 3 \mathrm{H}), 2.02-1.84(\mathrm{~m}, 1 \mathrm{H}), 1.80-1.07(\mathrm{~m}, 38 \mathrm{H})$, $1.07-0.70(\mathrm{~m}, 15 \mathrm{H}) .{ }^{13} \mathrm{C} \mathrm{NMR}\left(75 \mathrm{MHz}, \mathrm{CDCl}_{3}\right) \delta=167.5,141.9,139.7,139.1,138.9,137.7,136.2,133.7$, $130.9,126.8,125.2,123.2,122.1,121.7,121.6,37.7,32.1,31.98,30.2$, 29.8, 29.8, 29.5, 29.3, 29.1, 29.0, 27.4, $27.0,22.8,14.2,13.8,11.2$.

General procedure for the direct arylation protocol for the synthesis of oligomers. Selected example for the synthesis of compound 24. 4,7-dibromobenzo[c][1,2,5]thiadiazole $22(150 \mathrm{mg}, 0.51 \mathrm{mmol})$ was added to a solution of compound 18 (519 g, 1,02 mmol), $\mathrm{Pd}(\mathrm{OAc})_{2}$ (11 mg, $\left.0.05 \mathrm{mmol}\right), \mathrm{PPh}_{3}(26 \mathrm{mg}, 0.1 \mathrm{mmol}), \mathrm{K}_{2} \mathrm{CO}_{3}$ $(138 \mathrm{mg}, 1 \mathrm{mmol})$ in dry DMAc $(3 \mathrm{~mL})$ and then warmed to $110{ }^{\circ} \mathrm{C}$ with an oil bath for $24 \mathrm{~h}$. After TLC monitoring (eluent 7:3 petroleum ether : $\mathrm{DCM}, R_{\mathrm{f}}=0.39$ ), reaction solvent was removed under reduce pressure. Crude of reaction was purified by flash chromatography $\left(\mathrm{SiO}_{2}\right.$; eluent: petroleum ether : $\left.\mathrm{DCM} 8: 2\right)$ to give pure product as red solid (296 mg, 46\%).

diicosyl 2,2'-(benzo[c][1,2,5]thiadiazole-4,7-diyl)bis(benzo[b]thieno[2,3-e]benzothiophene-4-carboxylate) (24). Red solid (296 mg, 46\%). ${ }^{1} \mathrm{H}$ NMR (400 MHz, $\left.\mathrm{CDCl}_{3}\right) \delta=9.22(\mathrm{~s}, 2 \mathrm{H}), 8.27$ (d, J=7.2 Hz, 2H), 8.16 $(\mathrm{d}, J=4.9 \mathrm{~Hz}, 2 \mathrm{H}), 7.81-7.73(\mathrm{~m}, 2 \mathrm{H}), 7.71(\mathrm{~s}, 2 \mathrm{H}), 7.52-7.37(\mathrm{~m}, 4 \mathrm{H}), 4.33-4.25(\mathrm{~m}, 4 \mathrm{H}), 1.98-1.86$ $(\mathrm{m}, 2 \mathrm{H}), 1.63-1.14(\mathrm{~m}, 64 \mathrm{H}), 0.93-0.78(\mathrm{~m}, 12 \mathrm{H}) .{ }^{13} \mathrm{C} \mathrm{NMR}\left(101 \mathrm{MHz}, \mathrm{CDCl}_{3}\right) \delta=165.9,152.0,140.9$, 138.4, 136.7, 135.3, 133.6, 133.5, 131.4, 129.5, 127.0, 126.6, 125.7, 124.7, 124.5, 124.4, 122.6, 122.4, 68.1, 37.3, 31.8, 31.4, 30.0, 29.6, 29.2, 26.7, 22.5, 13.9. HRMS (MALDI-TOF): Calcd. for $\mathrm{C}_{76} \mathrm{H}_{96} \mathrm{~N}_{2} \mathrm{O}_{4} \mathrm{~S}_{5}$ : 1260.5974. Found: 1260.6020. 
bis(2-octyldodecyl) [2,2'-bibenzo[b]thieno[2,3-e]benzothiophene]-4,4'-dicarboxylate (28). Pale yellow oil (23 $\mathrm{mg}, 4 \%) .{ }^{1} \mathrm{H} \mathrm{NMR}\left(200 \mathrm{MHz}, \mathrm{CDCl}_{3}\right) \delta=8.65(\mathrm{~s}, 2 \mathrm{H}), 8.35(\mathrm{~d}, J=5.6 \mathrm{~Hz}, 4 \mathrm{H}), 7.85(\mathrm{~d}, J=7.5 \mathrm{~Hz}, 2 \mathrm{H}), 7.61$ $-7.42(\mathrm{~m}, 4 \mathrm{H}), 4.35(\mathrm{~d}, J=5.5 \mathrm{~Hz}, 4 \mathrm{H}), 1.91(\mathrm{~d}, J=5.4 \mathrm{~Hz}, 2 \mathrm{H}), 1.26(\mathrm{~s}, 64 \mathrm{H}), 0.87$ (dd, $J=10.3,4.3 \mathrm{~Hz}$, $12 \mathrm{H})$.

diicosyl 2,2'-(benzo[c][1,2,5]thiadiazole-4,7-diyl)bis(benzo[b]thieno[3,2-g]benzothiophene-4-carboxylate) (26). Red solid (264 mg, 41\%). ${ }^{1} \mathrm{H}$ NMR (400 MHz, $\left.\mathrm{CDCl}_{3}\right) \delta=8.89$ (s, 2H), 8.27 (s, 2H), 7.65 (d, J=7.8 Hz, 4H), $7.48(\mathrm{~s}, 2 \mathrm{H}), 7.28-7.19(\mathrm{t}, J=7.4 \mathrm{~Hz}, 2 \mathrm{H}), 7.13(\mathrm{t}, J=7.4 \mathrm{~Hz}, 2 \mathrm{H}), 4.45$ (d, $J=5.8 \mathrm{~Hz}, 4 \mathrm{H}), 2.08-1.96$ $(\mathrm{m}, 2 \mathrm{H}), 1.69-1.14(\mathrm{~m}, 64 \mathrm{H}), 0.93-0.79(\mathrm{~m}, 12 \mathrm{H}) .{ }^{13} \mathrm{C} \mathrm{NMR}\left(101 \mathrm{MHz}, \mathrm{CDCl}_{3}\right) \delta=166.9,152.3,139.7$, 139.1, 137.7, 137.2, 135.9, 135.3, 132.0, 126.9, 126.1, 125.8, 125.8, 125.1, 123.0, 122.0, 121.9, 121.8, 68.6, 38.2, 32.5, 32.4, 32.2, 30.7, 30.3, 29.9, 27.5, 23.2, 14.6. HRMS (MALDI-TOF): calcd. $\mathrm{C}_{76} \mathrm{H}_{96} \mathrm{~N}_{2} \mathrm{O}_{4} \mathrm{~S}_{5}$ : 1260.5974. Found: 1260.5976.

bis(2-octyldodecyl) [2,2'-bibenzo[b]thieno[3,2-g]benzothiophene]-4,4'-dicarboxylate (29). Pale yellow oil $(11 \mathrm{mg}, 2 \%) .{ }^{1} \mathrm{H}$ NMR $\left(300 \mathrm{MHz}, \mathrm{CDCl}_{3}\right) \delta=8.64(\mathrm{~s}, 2 \mathrm{H}), 8.46(\mathrm{~s}, 2 \mathrm{H}), 8.00(\mathrm{~d}, J=3.8 \mathrm{~Hz}, 2 \mathrm{H}), 7.77(\mathrm{~d}, J=$ $5.0 \mathrm{~Hz}, 2 \mathrm{H}), 7.44-7.32(\mathrm{~m}, 4 \mathrm{H}), 4.45(\mathrm{~d}, J=5.6 \mathrm{~Hz}, 4 \mathrm{H}), 2.05-1.89(\mathrm{~m}, 2 \mathrm{H}), 1.64-1.16(\mathrm{~m}, 58 \mathrm{H}), 0.83(\mathrm{t}$, $J=6.3 \mathrm{~Hz}, 12 \mathrm{H}) .{ }^{13} \mathrm{C}$ NMR $\left(75 \mathrm{MHz}, \mathrm{CDCl}_{3}\right) \delta=166.72,138.83,137.86,137.26,135.70,134.43,132.30$, 127.10, 127.04, 125.16, 123.97, 122.95, 122.11, 121.93, 68.43, 37.86, 32.10, 32.06, 31.92, 30.30, 29.92, 29.87, $29.56,29.52,27.18,22.83,14.25$.

General procedure for the Stille protocol for the synthesis of oligomers. Synthesis of compound 25. A solution of icosyl 2-(tributylstannyl)benzo[b]thieno[2,3-e]benzothiophene-4-carboxylate 19 (118 mg, 0.21 mmol) in $1 \mathrm{~mL}$ of degassed dry toluene was added to a degassed solution of 4,7-dibromo-5,6difluorobenzo[c][1,2,5]thiadiazole $23(33 \mathrm{mg}, 0.1 \mathrm{mmol}), \operatorname{Pd}\left(\mathrm{PPh}_{3}\right)_{4}(4.5 \mathrm{mg}, 0.04 \mathrm{mmol}, 0.05 \mathrm{eq})$, in dry toluene $(1 \mathrm{~mL})$ and then stirred to $110^{\circ} \mathrm{C}$ in an oil bath for $24 \mathrm{~h}$. After TLC monitoring $(7: 3$ petroleum ether : DCM, $\left.\mathrm{R}_{\mathrm{f}}=0.41\right)$, reaction solvent was removed under reduced pressure. The crude reaction mixture was purified by flash chromatography $\left(\mathrm{SiO}_{2}\right.$; eluent: petroleum ether : DCM 8:2). The pure product was obtained as orange solid (97 $\mathrm{mg}, 75 \%$ ).

diicosyl 2,2'-(5,6-difluorobenzo[c][1,2,5]thiadiazole-4,7-diyl)bis(benzo[b]thieno[2,3-e]benzothiophene-4carboxylate) (25). Red solid (97 mg, 75\%). ${ }^{1} \mathrm{H}$ NMR (400 MHz, $\left.\mathrm{CDCl}_{3}\right) \delta=9.12$ (s, 2H), 7.98 (d, $J=7.4 \mathrm{~Hz}$, 2H), $7.91(\mathrm{~d}, J=5.6 \mathrm{~Hz}, 2 \mathrm{H}), 7.57(\mathrm{~d}, J=7.4 \mathrm{~Hz}, 2 \mathrm{H}), 7.38-7.31(\mathrm{~m}, 2 \mathrm{H}), 7.26(\mathrm{t}, J=7.3 \mathrm{~Hz}, 2 \mathrm{H}), 4.26(\mathrm{~d}$, $J=5.2 \mathrm{~Hz}, 4 \mathrm{H}), 1.97-1.84(\mathrm{~m}, 2 \mathrm{H}), 1.61-1.17(\mathrm{~m}, 64 \mathrm{H}), 0.91-0.81(\mathrm{~m}, 12 \mathrm{H}) .{ }^{13} \mathrm{C} \mathrm{NMR}\left(101 \mathrm{MHz}, \mathrm{CDCl}_{3}\right)$ $\delta=166.1,148.3,141.2,136.4,136.1,135.8,133.9,131.5,131.4,128.8,128.7,126.9,125.0,124.9,123.1$, 123.0, 122.6, 111.7, 111.6, 68.8, 37.9, 32.5, 32.4, 31.9, 30.7, 30.3, 30.2, 29.9, 27.4, 23.2, 14.6. HRMS (MALDI-TOF): calcd for $\mathrm{C}_{76} \mathrm{H}_{94} \mathrm{~F}_{2} \mathrm{~N}_{2} \mathrm{O}_{4} \mathrm{~S}_{5}: 1296.5785$. Found: 1296.5808 .

diicosyl 2,2'-(5,6-difluorobenzo[c][1,2,5]thiadiazole-4,7-diyl)bis(benzo[b]thieno[3,2-g]benzothiophene-4carboxylate) (27). Red solid (104 mg, 80\%). ${ }^{1} \mathrm{H}$ NMR (400 MHz, $\left.\mathrm{CDCl}_{3}\right) \delta=8.86$ (s, 2H), 8.10 (s, 2H), 7.54 $(\mathrm{d}, J=7.7 \mathrm{~Hz}, 2 \mathrm{H}), 7.41(\mathrm{~d}, J=7.7 \mathrm{~Hz}, 2 \mathrm{H}), 7.10$ (t, $J=7.3 \mathrm{~Hz}, 2 \mathrm{H}), 6.91$ (t, $J=7.2 \mathrm{~Hz}, 2 \mathrm{H}), 4.46(\mathrm{~d}, J=5.3$ $\mathrm{Hz}, 4 \mathrm{H}), 2.10-1.90(\mathrm{~m}, 2 \mathrm{H}), 1.72-1.16(\mathrm{~m}, 64 \mathrm{H}), 0.88(\mathrm{~d}, J=7.4 \mathrm{~Hz}, 12 \mathrm{H}) .{ }^{13} \mathrm{C} \mathrm{NMR}\left(101 \mathrm{MHz}, \mathrm{CDCl}_{3}\right) \delta$ 
$=166.6,139.0,136.5,136.3,135.8,135.5,132.2,132.1,129.3,129.2,126.8,124.8,122.8,122.1,122.0,121.6$, 111.3, 68.7, 38.2, 32.5, 32.4, 32.1, 30.8, 30.3, 29.9, 27.5, 23.2, 14.6. HRMS (MALDI-TOF): calcd. for $\mathrm{C}_{76} \mathrm{H}_{94} \mathrm{~F}_{2} \mathrm{~N}_{2} \mathrm{O}_{4} \mathrm{~S}_{5}: 1296.5785$. Found: 1296.5833. 


\section{Additional experiments}

Compound 24

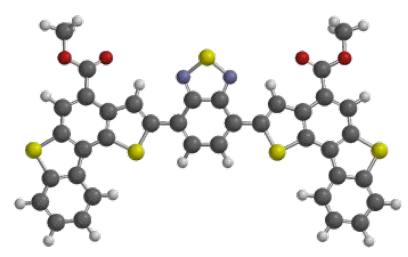

Compound 26

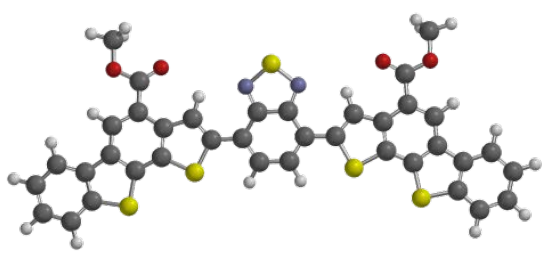

Compound 25

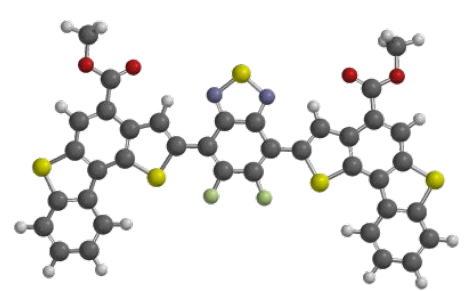

Compound 27

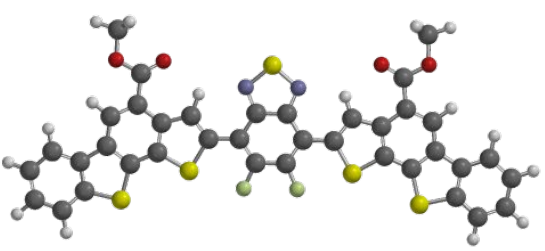

24 (1)

Figure S1. Computationally determined structures of the HOMO and LUMO for compounds 24-27. 


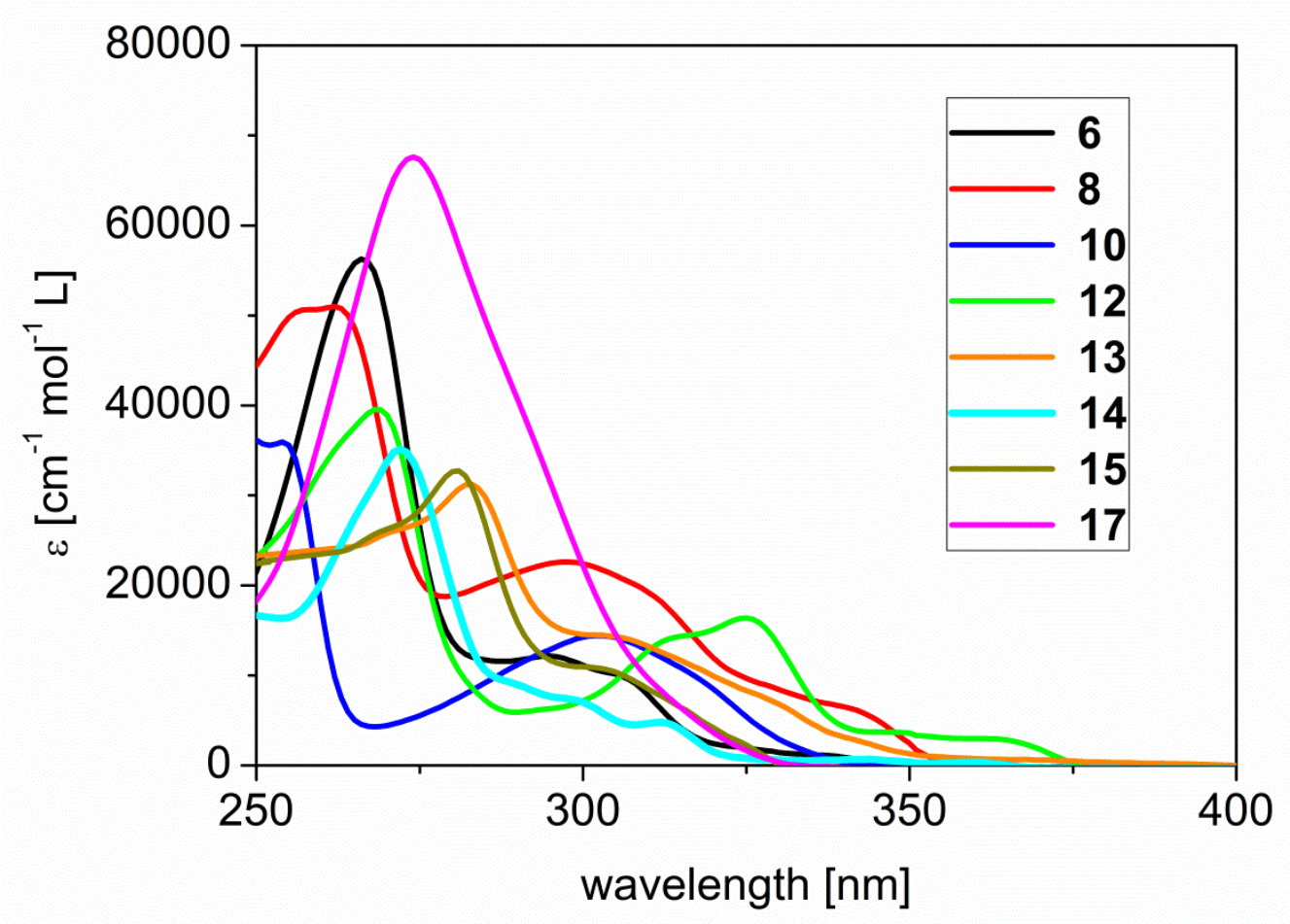

Figure S2. UV-Vis of compounds 6-17 in solution $10^{-5} \mathrm{~mol} \cdot \mathrm{L}^{-1}$ of $\mathrm{CHCl}_{3}$.

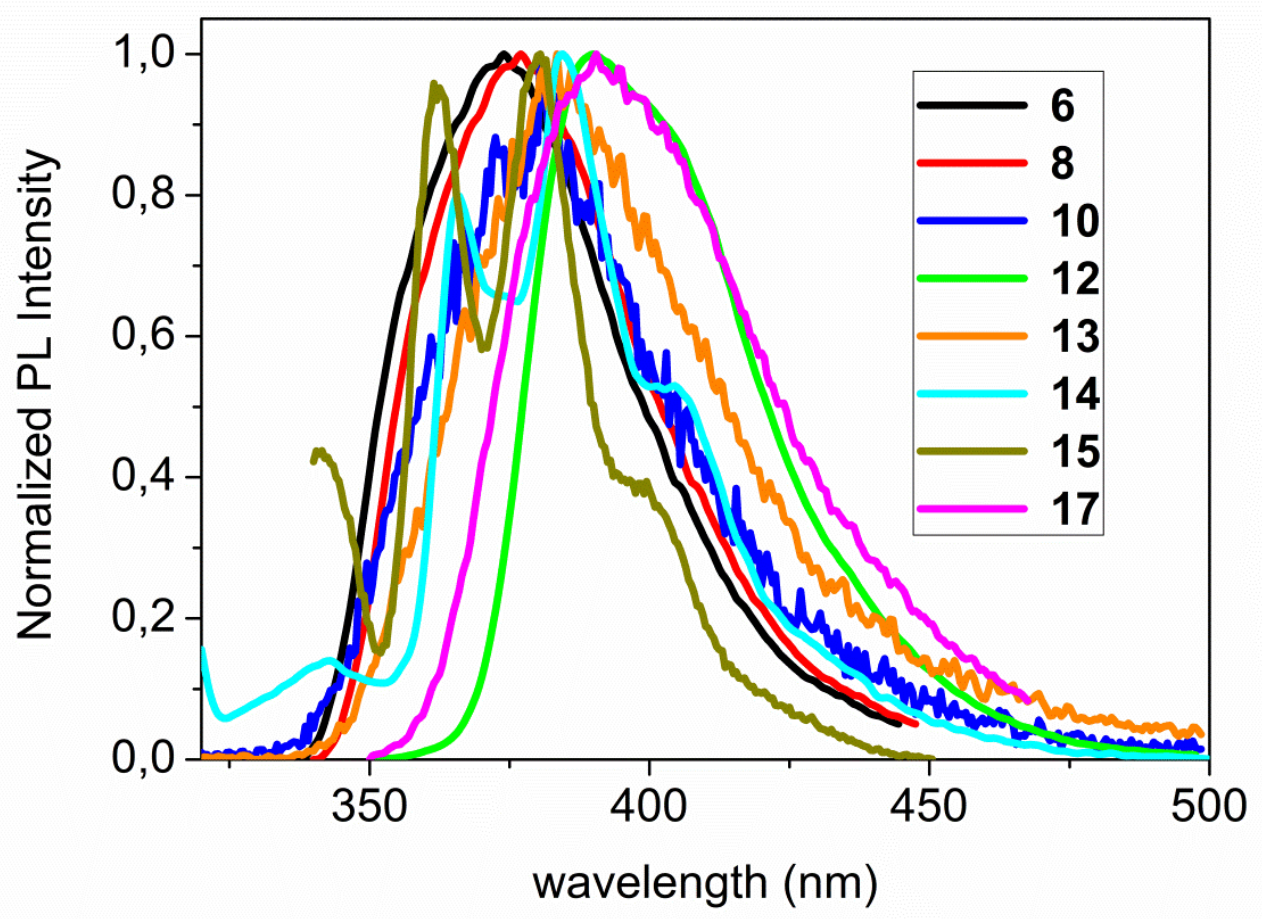

Figure S3. Normalized PL spectra of compound 6-17 in solution $10^{-5} \mathrm{~mol} \cdot \mathrm{L}^{-1}$ of $\mathrm{CHCl}_{3}$. 


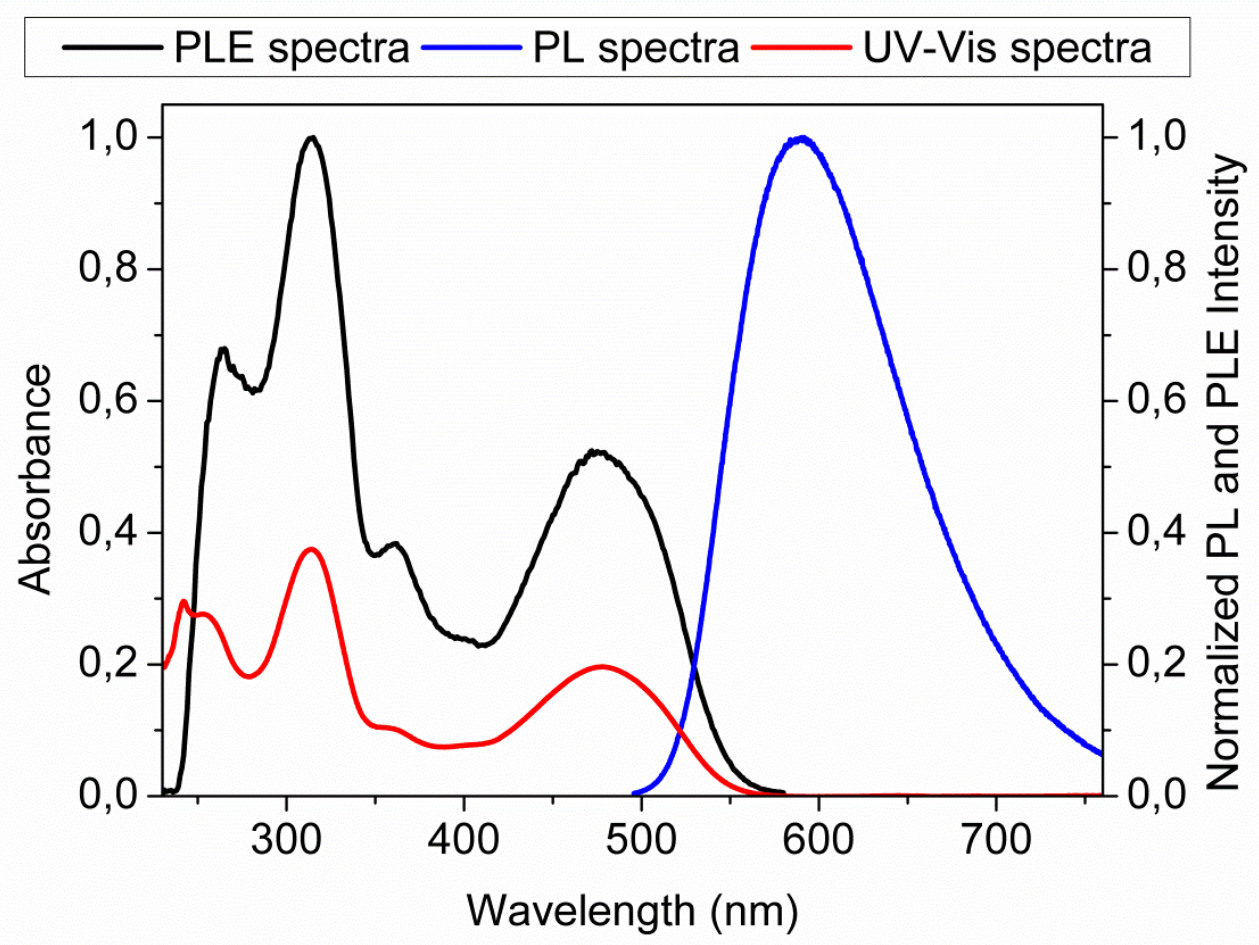

Figure S4. Spectroscopic characterization of compound 24 in $\mathrm{CHCl}_{3}$ solution $\left(10^{-5} \mathrm{~mol} \mathrm{~L}^{-1}\right)$. Red line: UVVis, Bue line: Normalized PL spectra, Black line: Normalized PLE spectra.

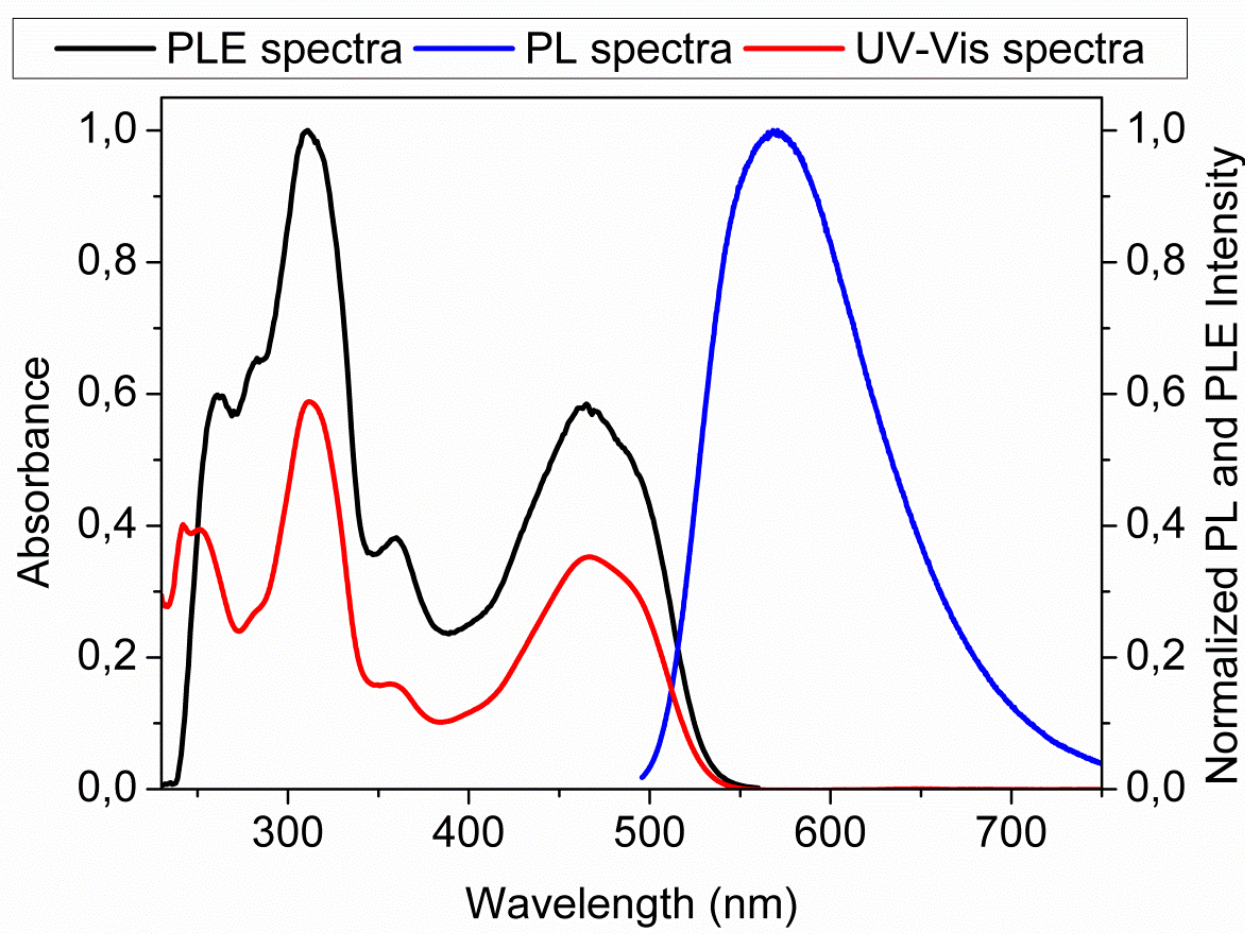

Figure S5. Spectroscopic characterization of compound 25 in $\mathrm{CHCl}_{3}$ solution $\left(10^{-5} \mathrm{~mol} \mathrm{~L}^{-1}\right)$. Red line: UVVis, Bue line: Normalized PL spectra, Black line: Normalized PLE spectra. 


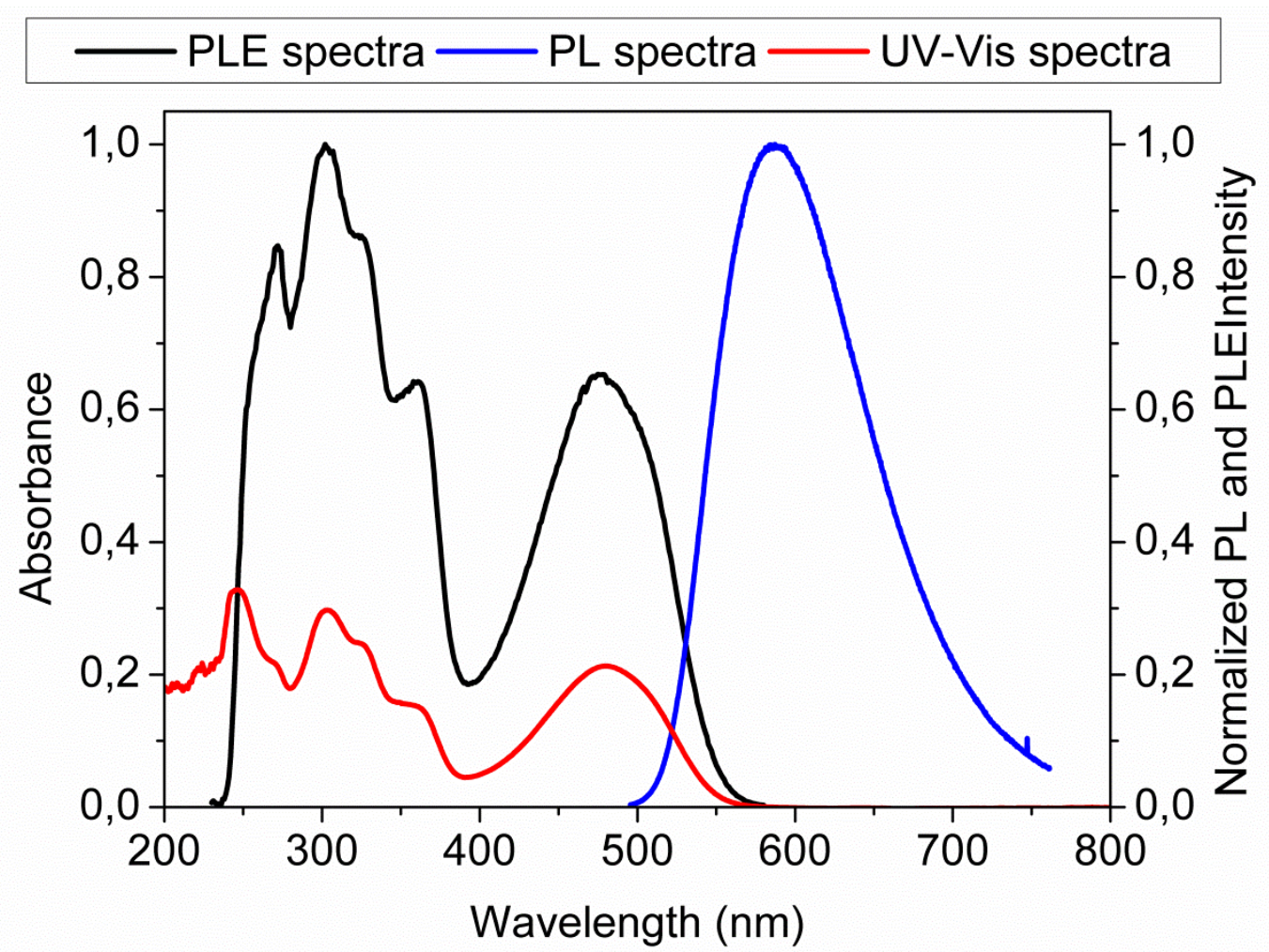

Figure S6. Spectroscopic characterization of compound 26 in $\mathrm{CHCl}_{3}$ solution $\left(10^{-5} \mathrm{~mol} \mathrm{~L}^{-1}\right)$. Red line: UVVis, Bue line: Normalized PL spectra, Black line: Normalized PLE spectra.

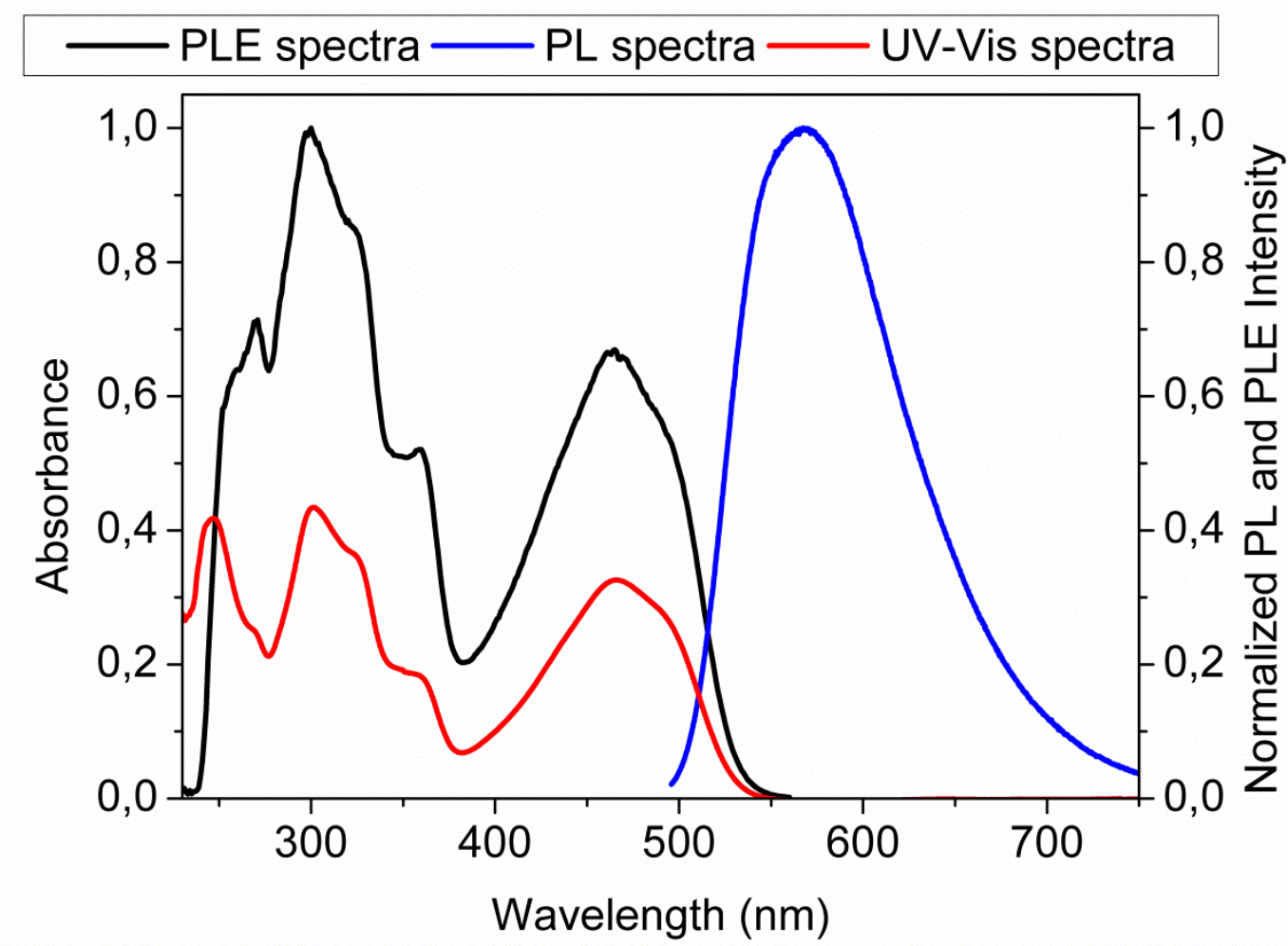

Figure S7. Spectroscopic characterization of compound 27 in $\mathrm{CHCl}_{3}$ solution $\left(10^{-5} \mathrm{~mol} \mathrm{~L}^{-1}\right)$. Red line: UVVis, Bue line: Normalized PL spectra, Black line: Normalized PLE spectra. 


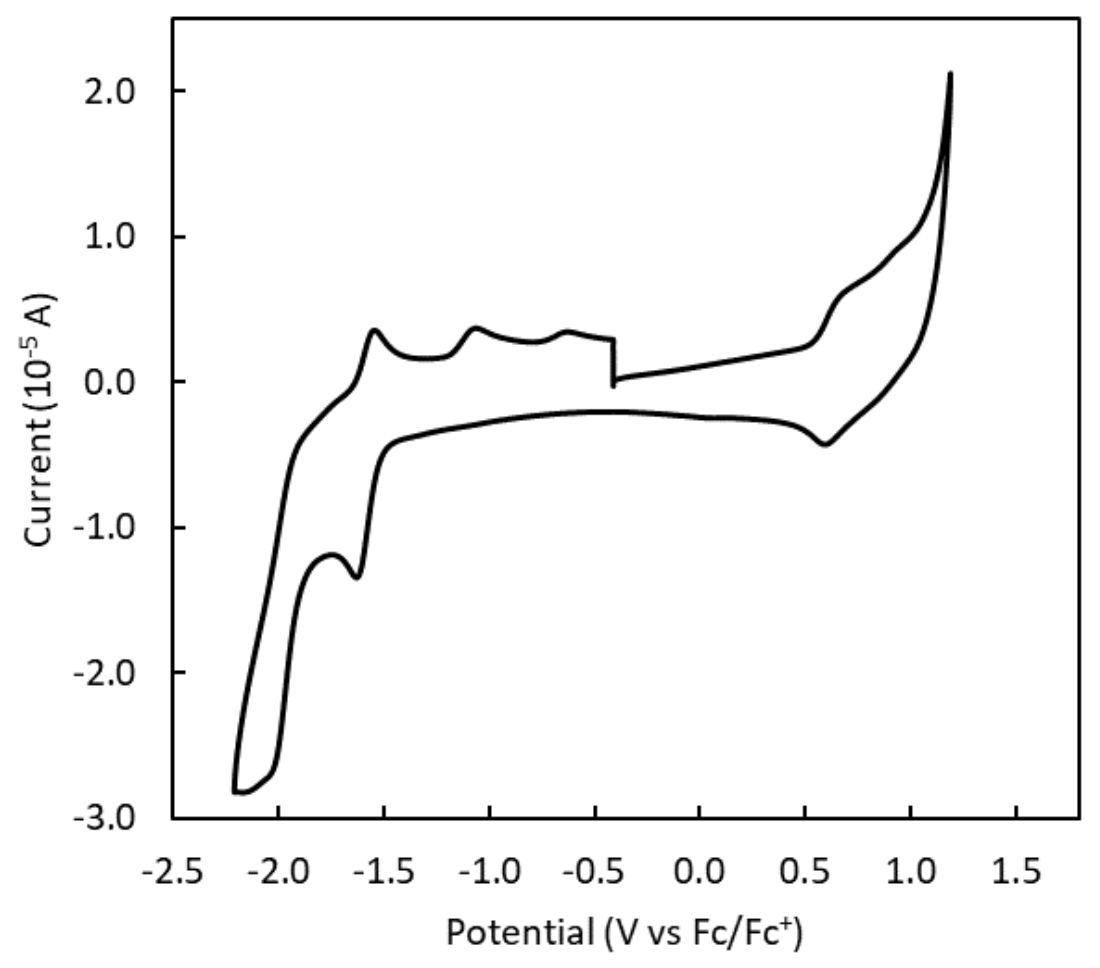

Figure S8. Cyclic Voltammogram of 24 in $0.1 \mathrm{M} \mathrm{TBAPF}_{6} \mathrm{CH}_{2} \mathrm{Cl}_{2}$.

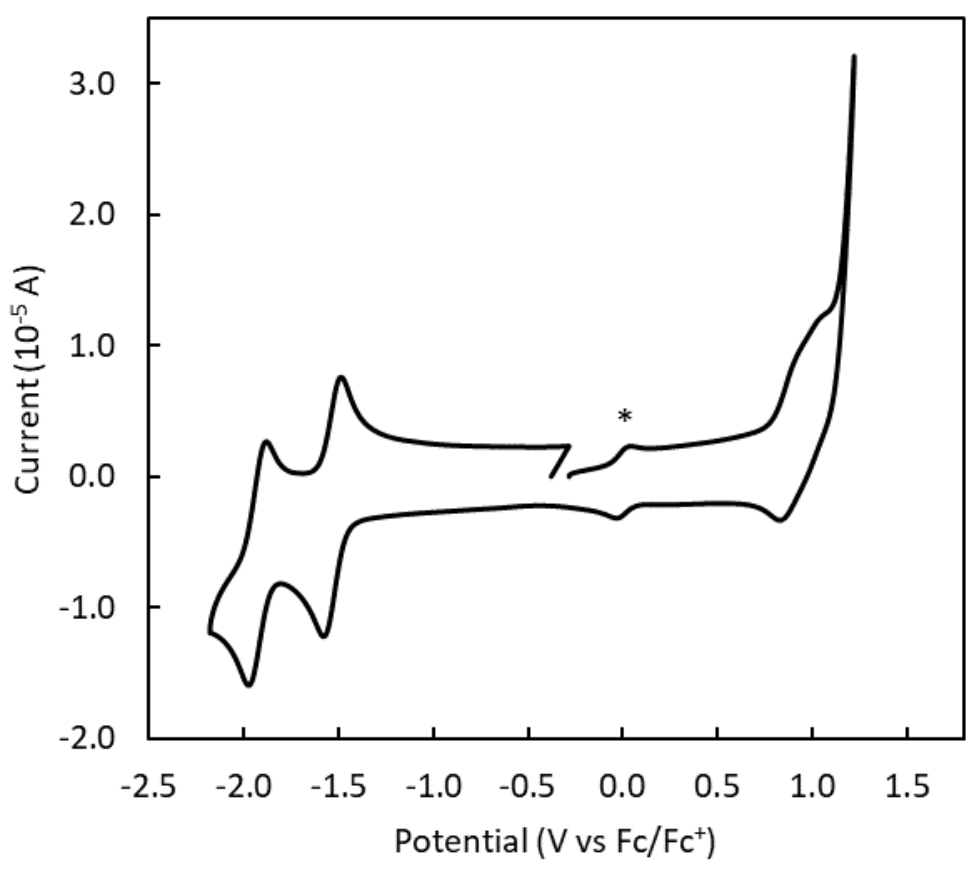

Figure S9. Cyclic Voltammogram of 25 in $0.1 \mathrm{M} \mathrm{TBAPF}_{6}$ in $1: 4 \mathrm{MeCN}: o-\mathrm{DCB}$. The asterisk $\left(^{*}\right)$ denotes the $\mathrm{Fc} / \mathrm{Fc}^{+}$redox couple. 


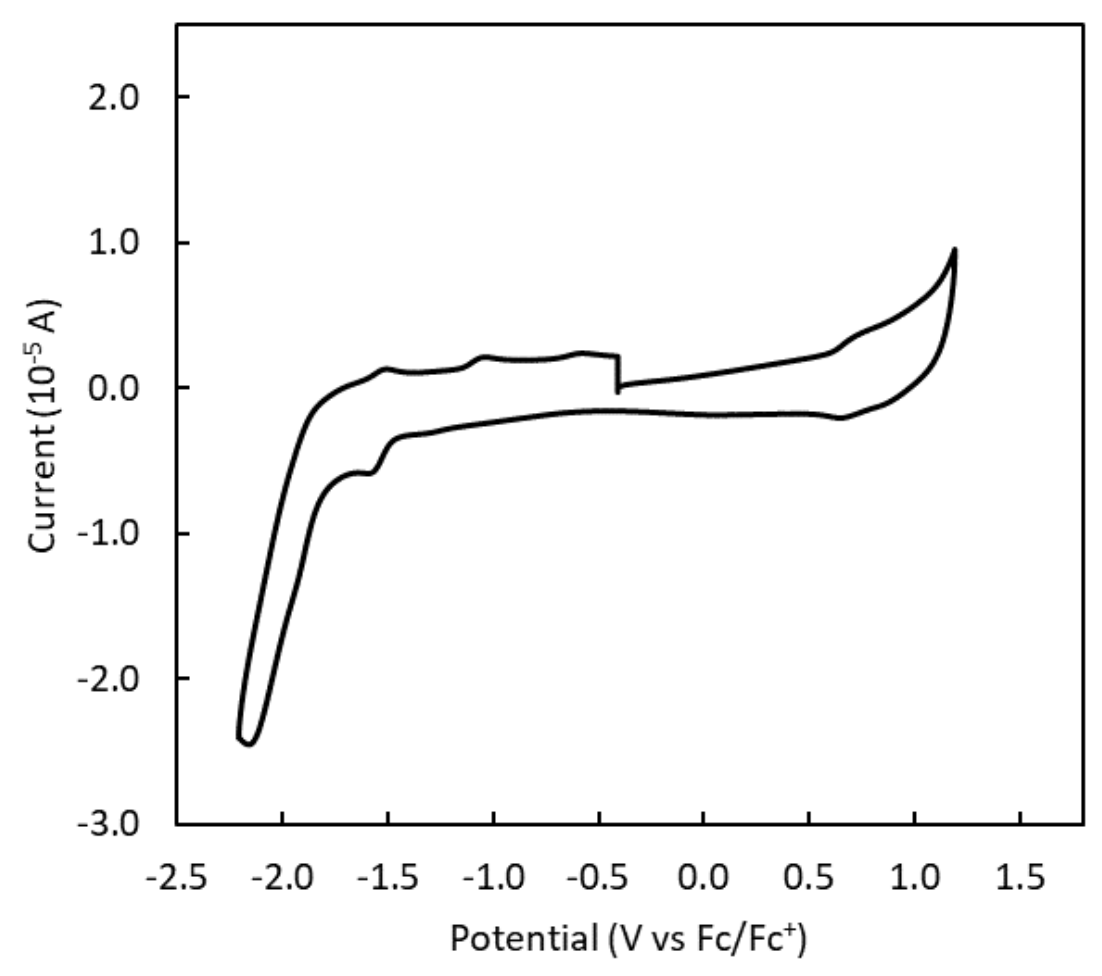

Figure S10. Cyclic Voltammogram of 26 in $0.1 \mathrm{M} \mathrm{TBAPF}_{6}$ in $\mathrm{CH}_{2} \mathrm{Cl}_{2}$.

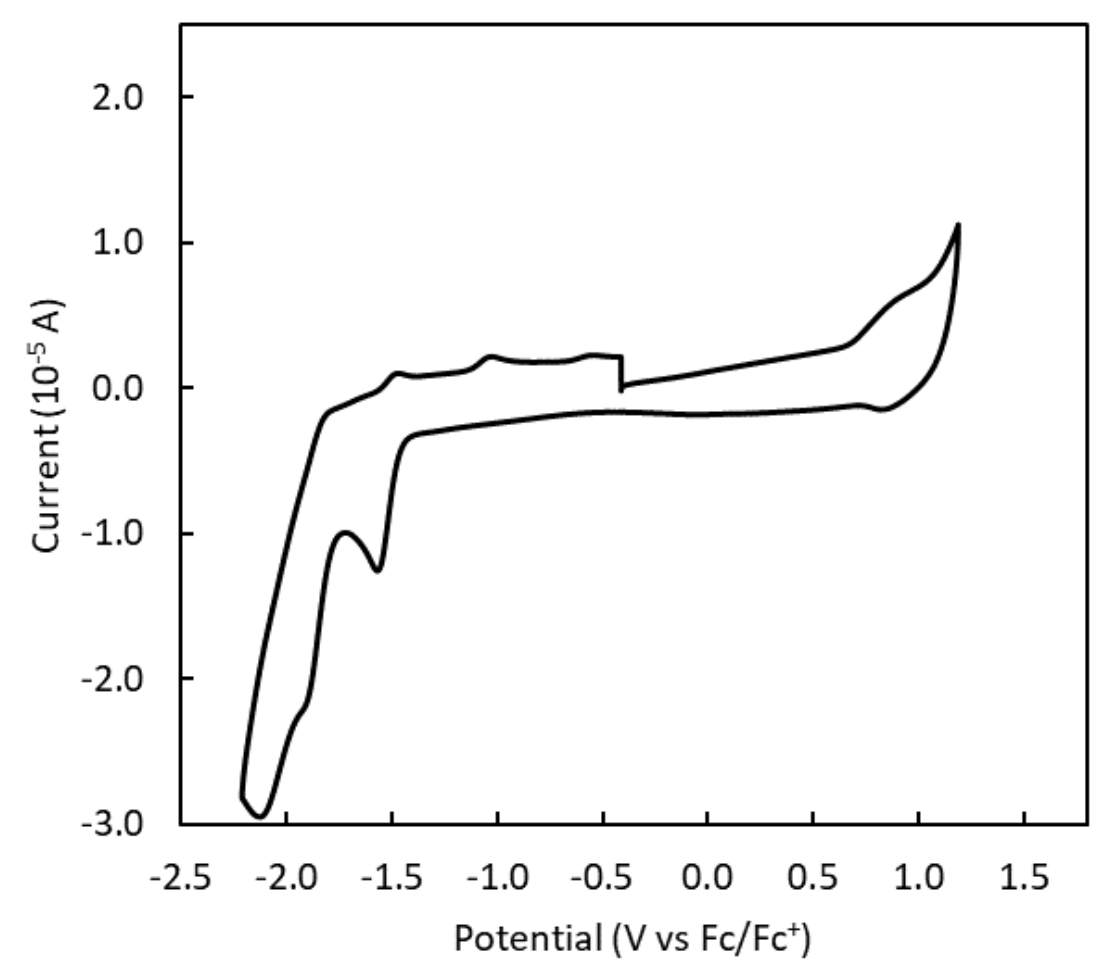

Figure S11. Cyclic Voltammogram of 27 in $0.1 \mathrm{M} \mathrm{TBAPF}_{6}$ in $\mathrm{CH}_{2} \mathrm{Cl}_{2}$. 


\section{References}

[1] Pommerehne, J.; Vestweber, H.; Guss, W.; Mahrt, R. F.; Bässler, H.; Porsch, M.; Daub, J. Adv. Mater. 1995, 7, 551-554.

[2] Burroughs, L.; Eccleshare, L.; Ritchie, Jo.; Kulkarni, O.; Lygo, B.; Woodward, S.; Lewis, W. Angew. Chem. Int. Ed. 2015, 54, 10648-10651.

[3] O. Hall, A.; Lee, S. R.; Bootsma, A. N.; Bloom, J. W. G.; Wheeler, S. E.; McNeil, A. J. J. Polym Sci Part A Polym Chem. 2017, 55, 1530-1535.

[4] Sankar, E.; Raju, P.; Karunakaran, J.; Mohanakrishnan, A. K. J. Org. Chem. 2017, 82, 13583-13593.

[5] Levine, D. R.; Siegler, M. A.; Tovar, J. D. J. Am. Chem. Soc. 2014, 136, 7132-7139. 


\section{Spectra of New Compounds}

Compound 8

${ }^{1} \mathrm{HNMR}\left(300 \mathrm{MHz}, \mathrm{CDCl}_{3}\right)$

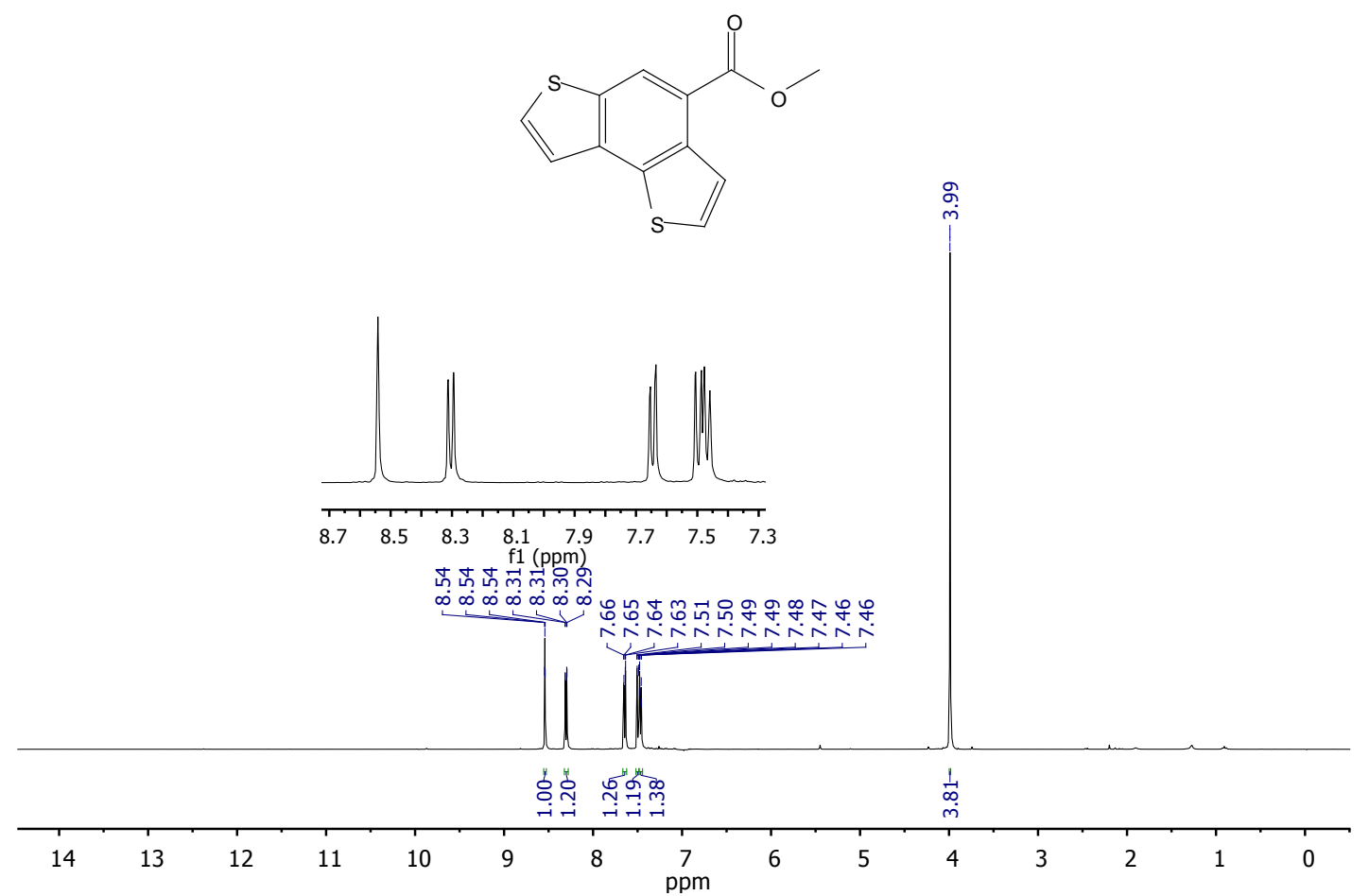

${ }^{13} \mathrm{CNMR}\left(90 \mathrm{MHz}, \mathrm{CDCl}_{3}\right)$

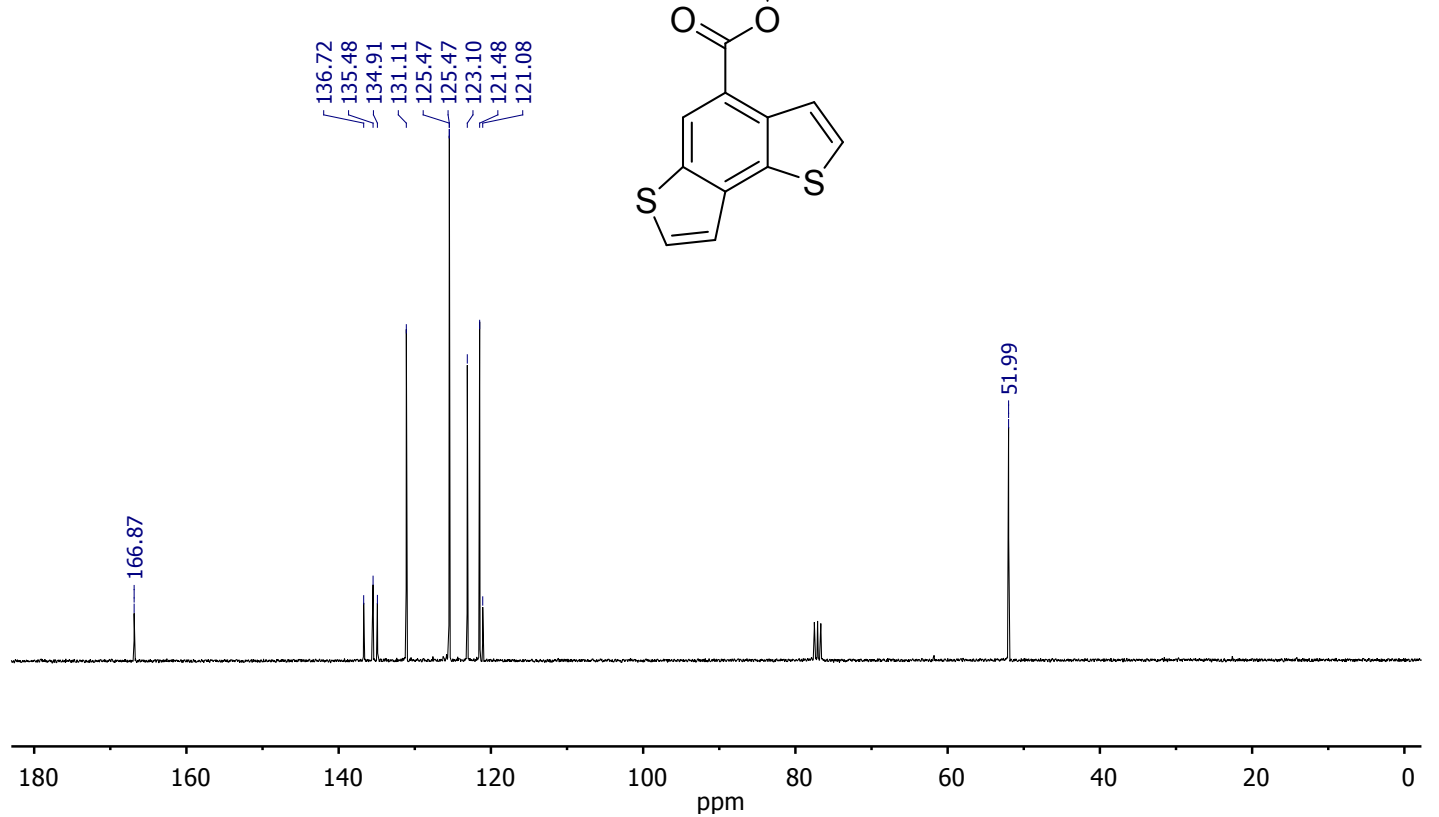




\section{DE-MS}

pasini09 \#3 RT: $0.07 \quad$ AV: 1 SB: 126 0.55-0.87, 1.02-2.30 NL: $1.24 E 7$
T: + c Full ms [50.00-1000.00]

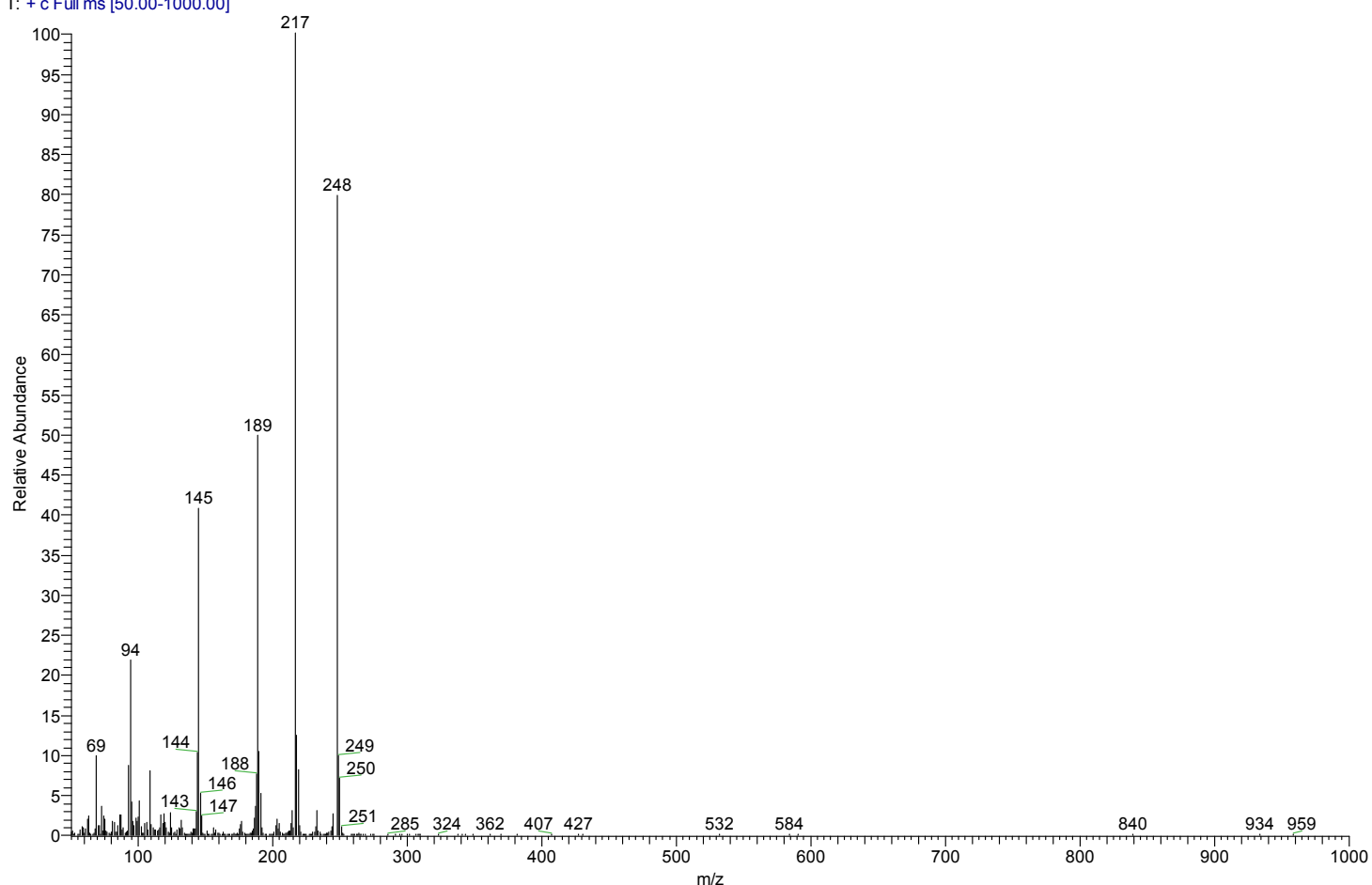


Compound 9

${ }^{1} \mathrm{H} N M R\left(300 \mathrm{MHz}, \mathrm{DMSO}-d_{6}\right)$

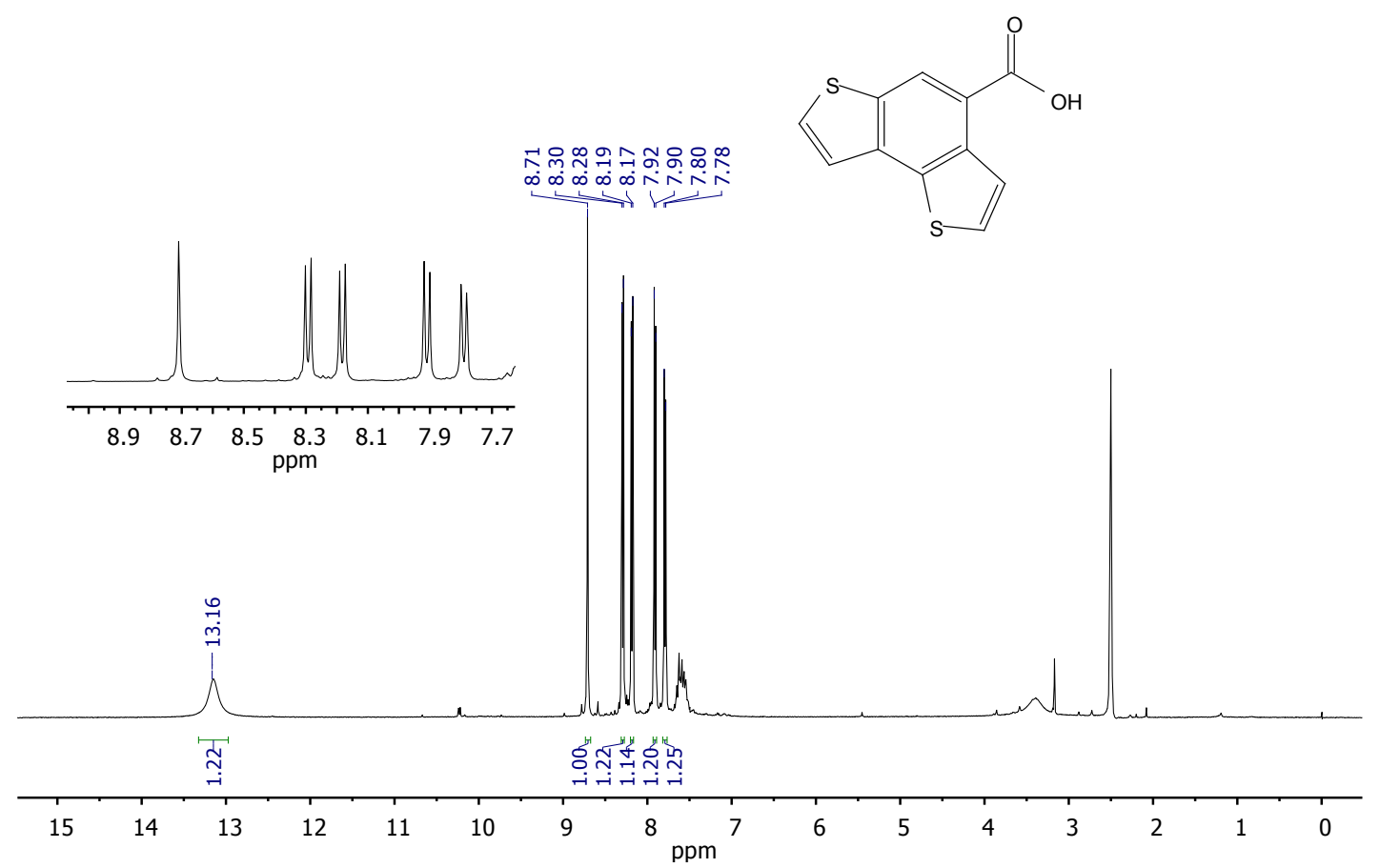

${ }^{13} \mathrm{C} N M R\left(90 \mathrm{MHz}, \mathrm{DMSO}-d_{6}\right)$<smiles>O=C(O)c1cc2sccc2c2sccc12</smiles>

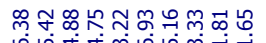

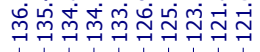
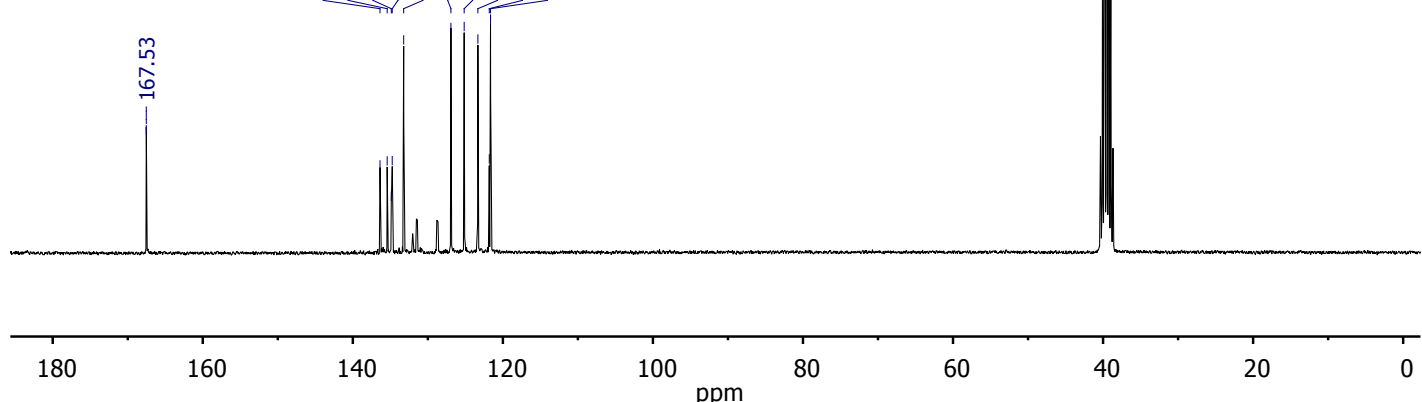


\section{ESI-MS}

an430ionineg \#1 RT: $0.00 \quad$ AV: 1 NL: $3.19 E 4$
T: TMS - c ESI Full ms $[50.00-1500.00]$

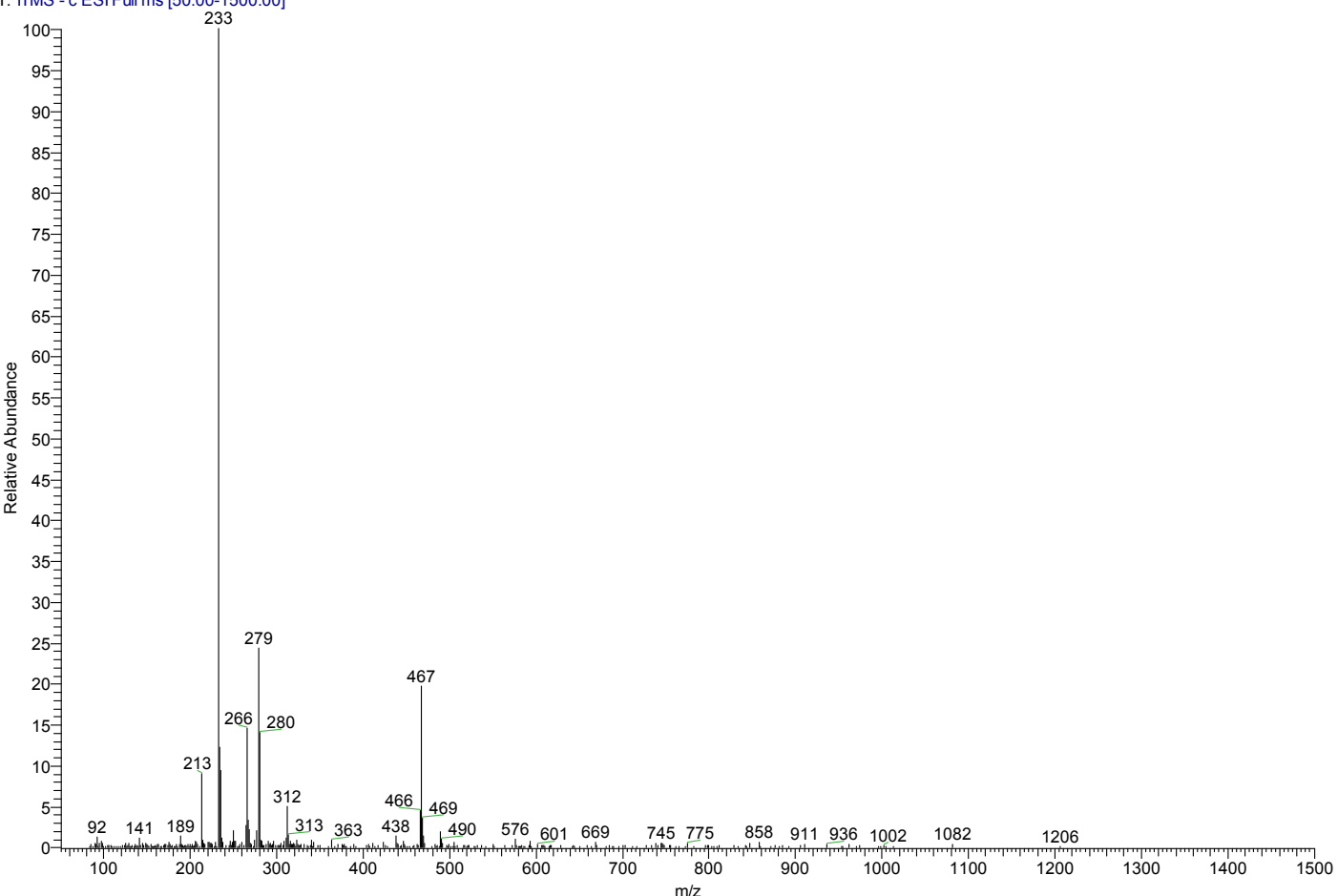




\section{Compound 10}

${ }^{1} \mathrm{HNMR}\left(300 \mathrm{MHz}, \mathrm{CDCl}_{3}\right)$<smiles>COC(=O)c1cc2occc2c2sccc12</smiles>
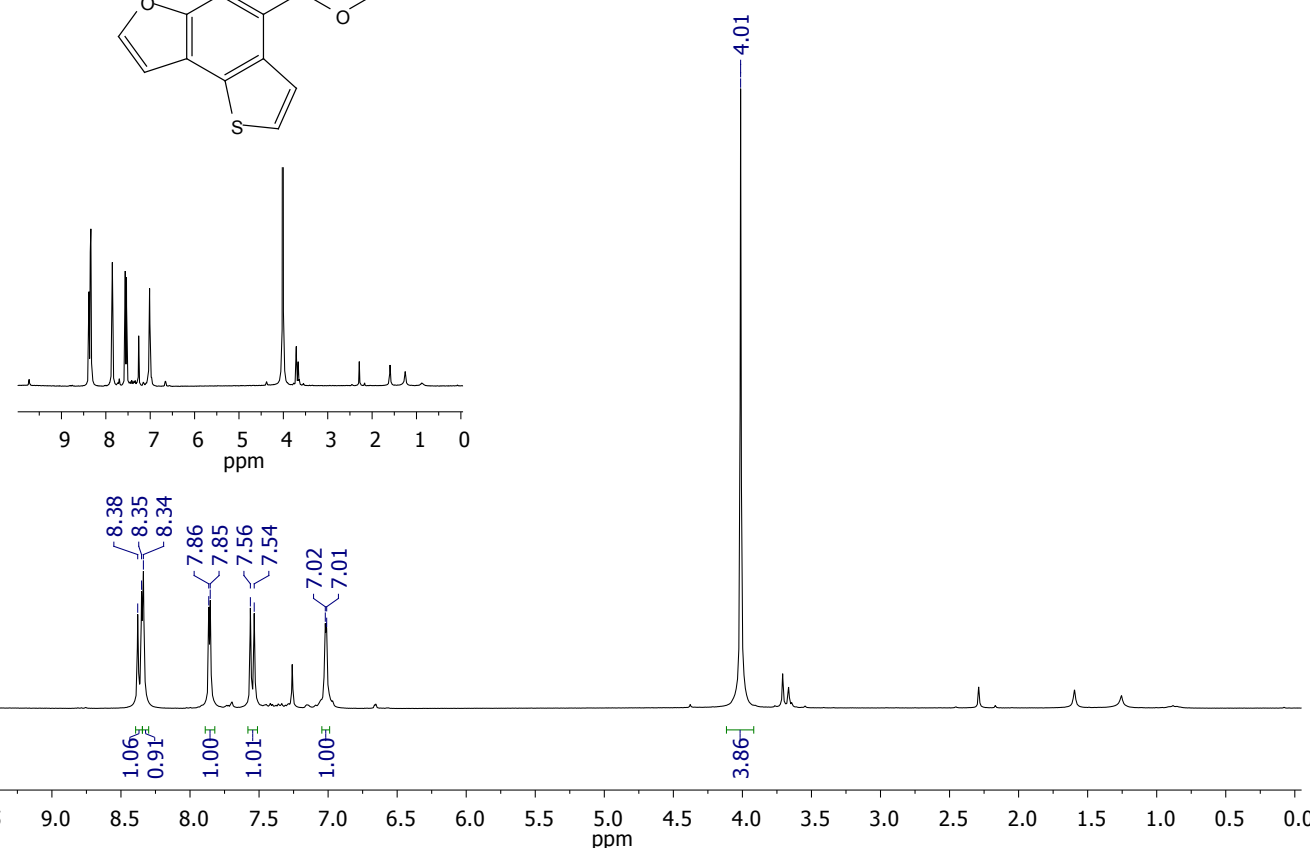

${ }^{13} \mathrm{C} \mathrm{NMR}\left(75 \mathrm{MHz}, \mathrm{CDCl}_{3}\right)$<smiles>COC(=O)c1cc2occc2c2sccc12</smiles>

$$
\text { rpm }
$$

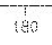

160

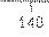

120

अ०

co

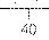




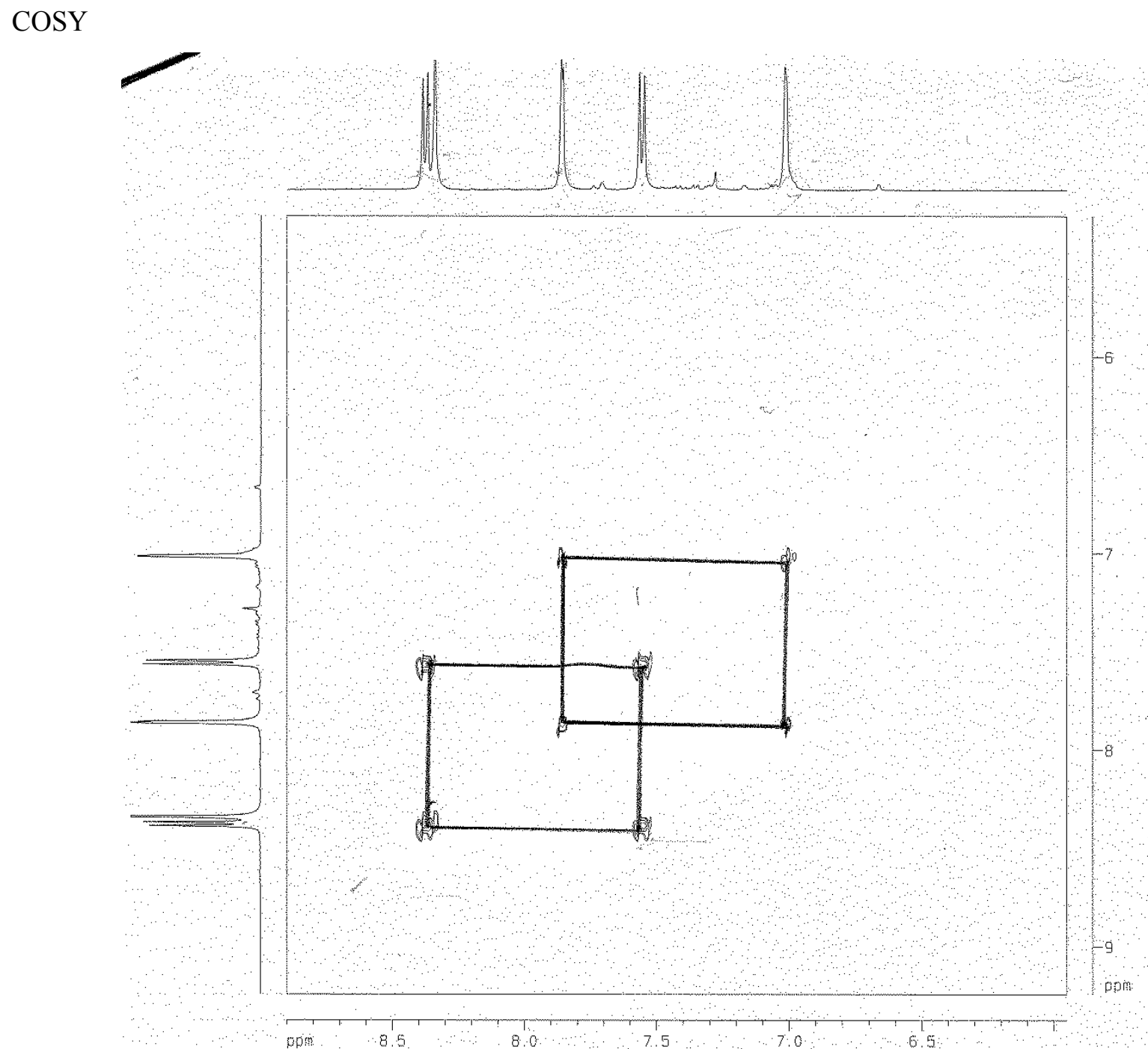

DE-MS

M193.206.72.116ldl...Pasinilpasini54

18/07/2018 11:58:59

GC 1

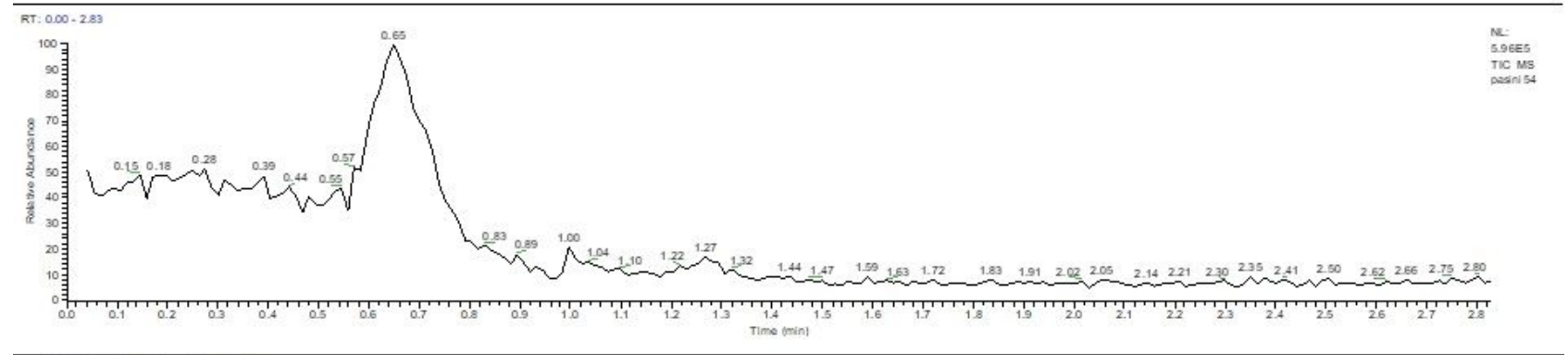

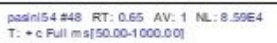

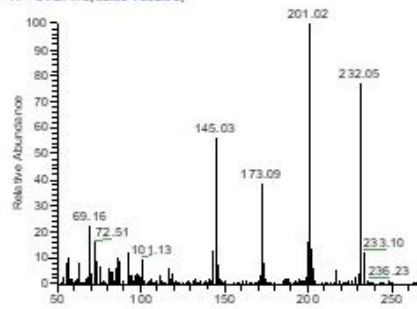

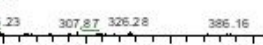

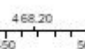
$\frac{14.3660 .87}{14}$

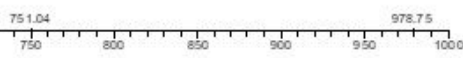


Compound 11

${ }^{1} \mathrm{H} N M R\left(200 \mathrm{MHz}, \mathrm{DMSO}-d_{6}\right)$

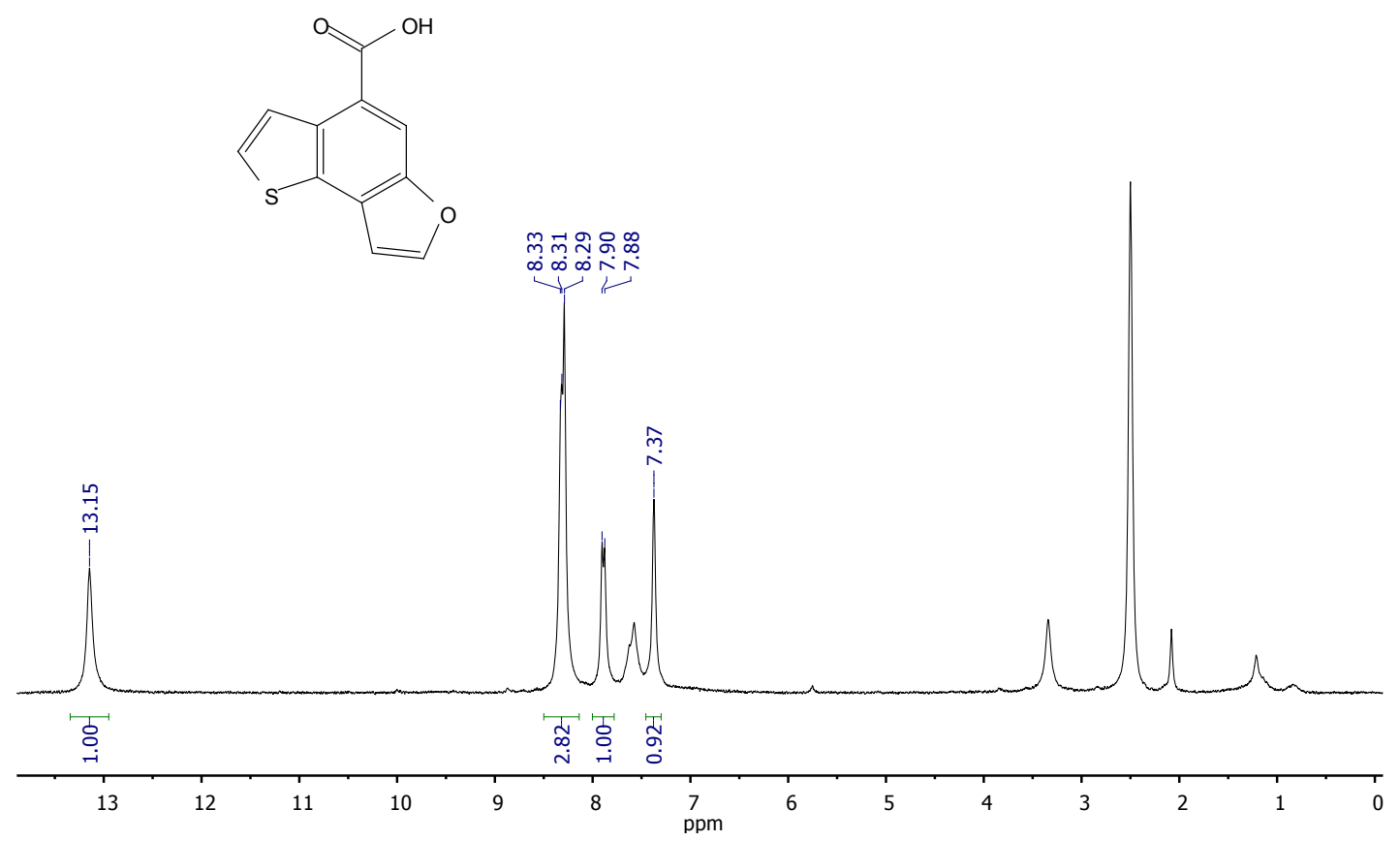

${ }^{13} \mathrm{C} N M R\left(90 \mathrm{MHz}, \mathrm{DMSO}-d_{6}\right)$

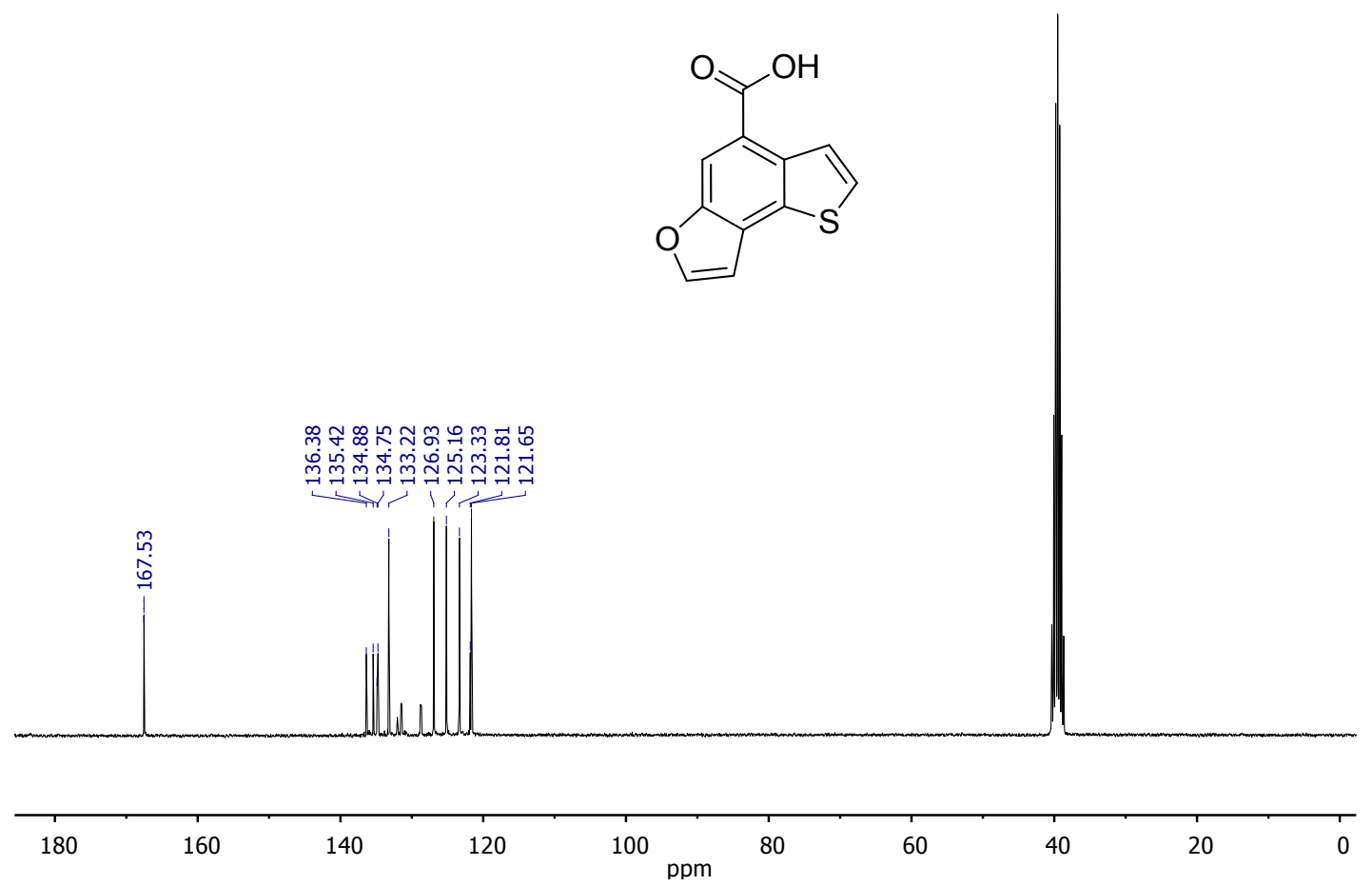




\section{ESI-MS}

an430ionineg \#1 RT: $0.00 \quad$ AV: $1 \quad$ NL: $3.19 E 4$
T: TMS - c ESIFull ms [50.00-1500.00]

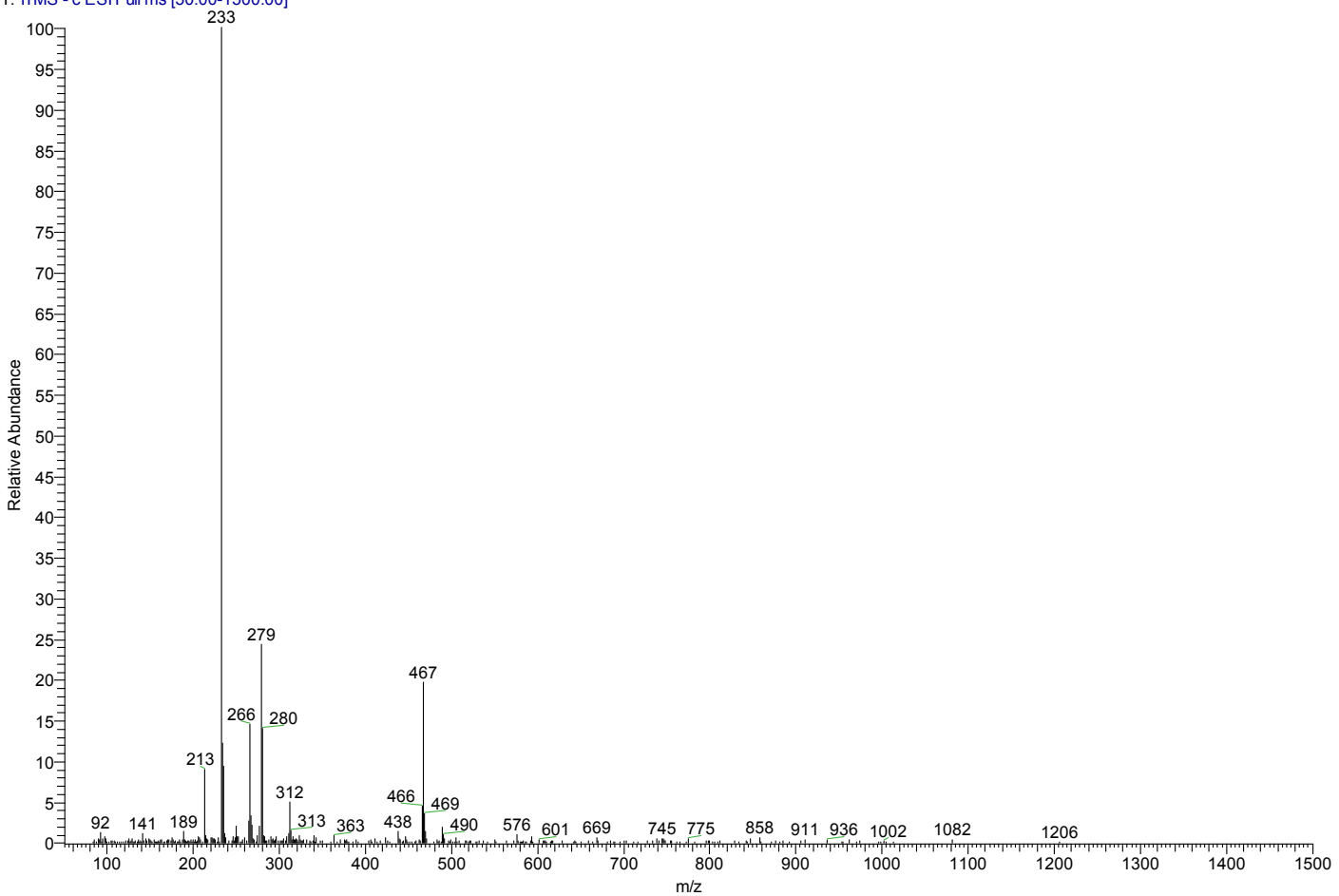




\section{Compound 12}

${ }^{1} \mathrm{H} \mathrm{NMR}\left(300 \mathrm{MHz}, \mathrm{CDCl}_{3}\right)$

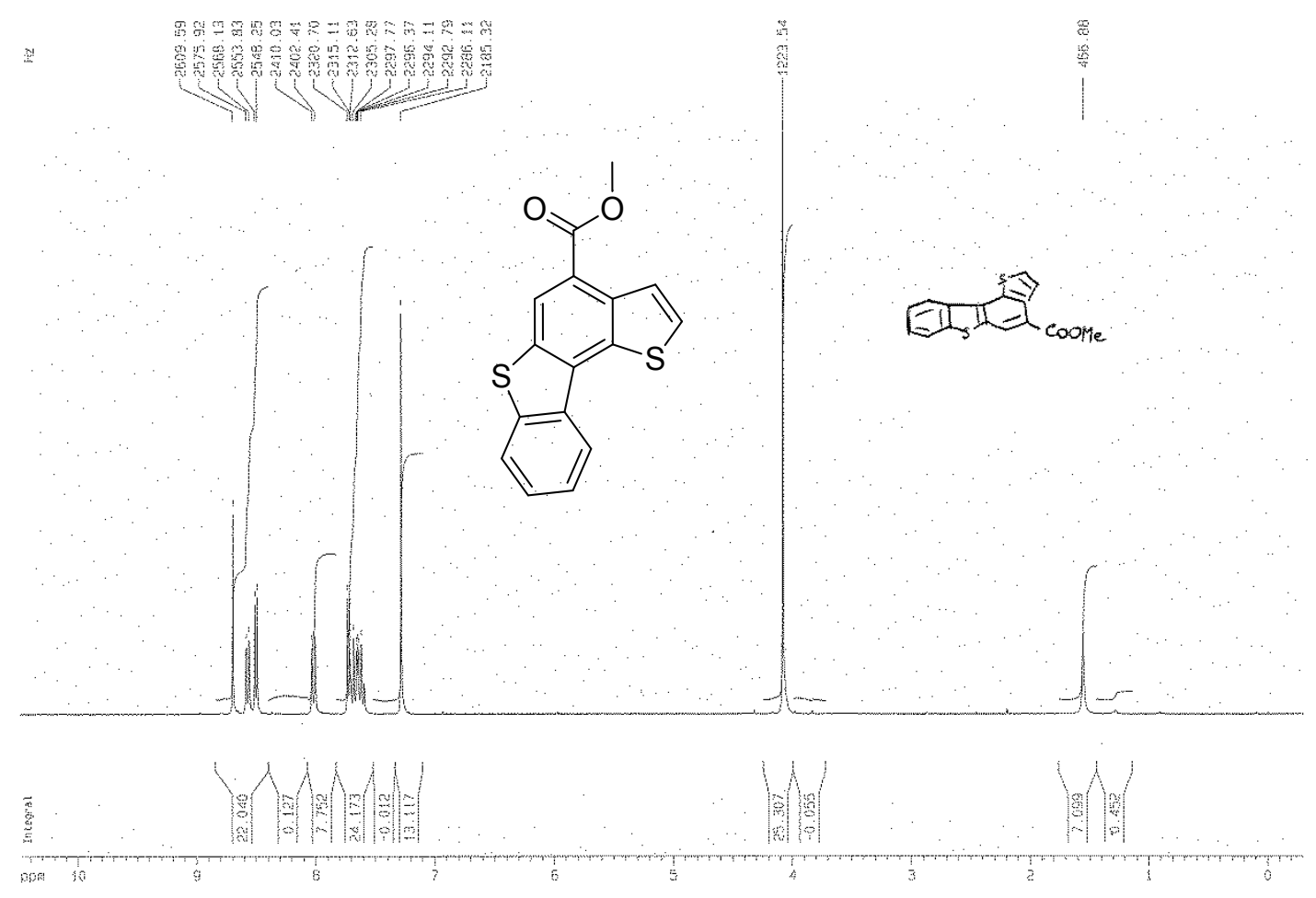

${ }^{13} \mathrm{C} \mathrm{NMR}\left(75 \mathrm{MHz}, \mathrm{CDCl}_{3}\right)$
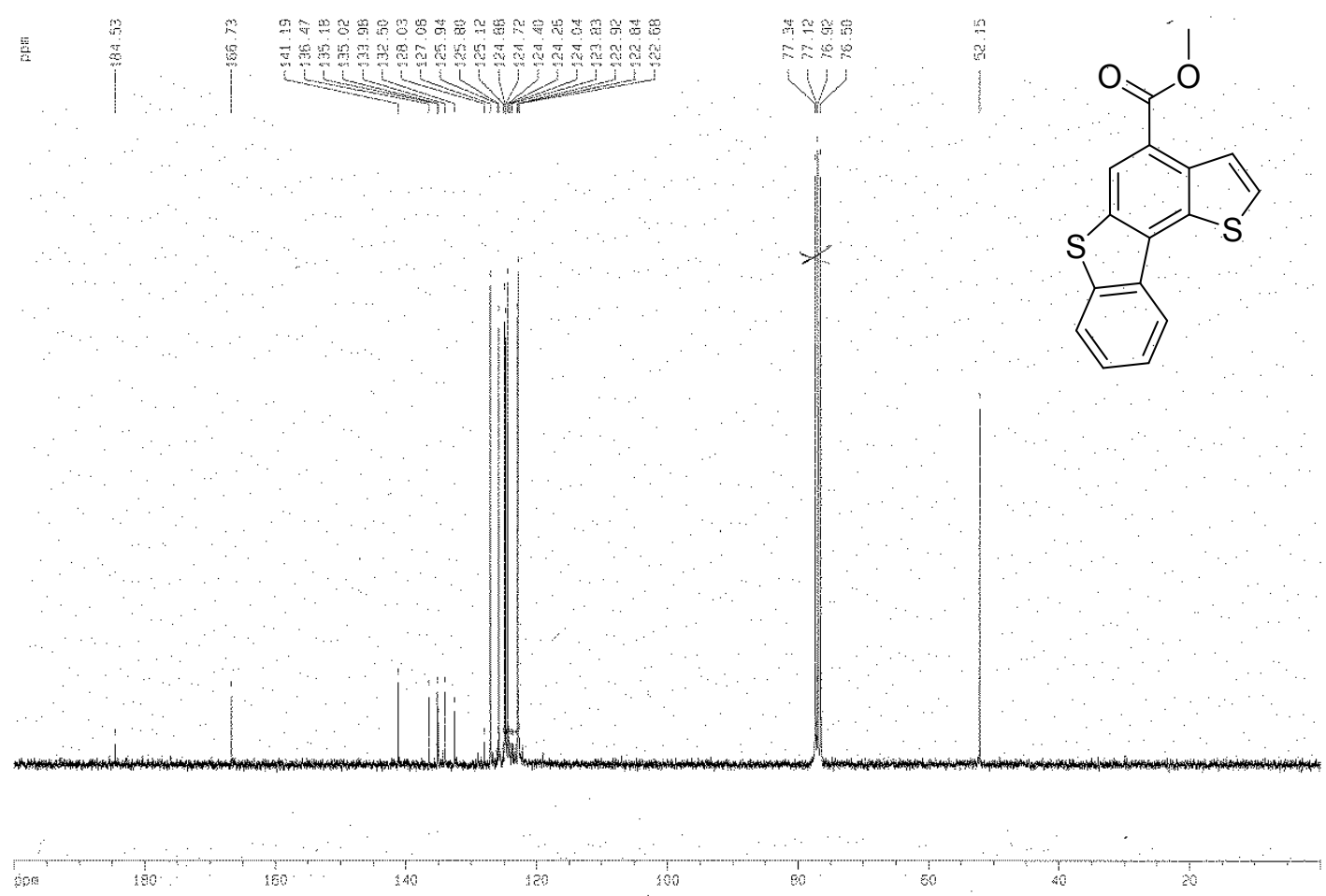
Zoom of aromatic region
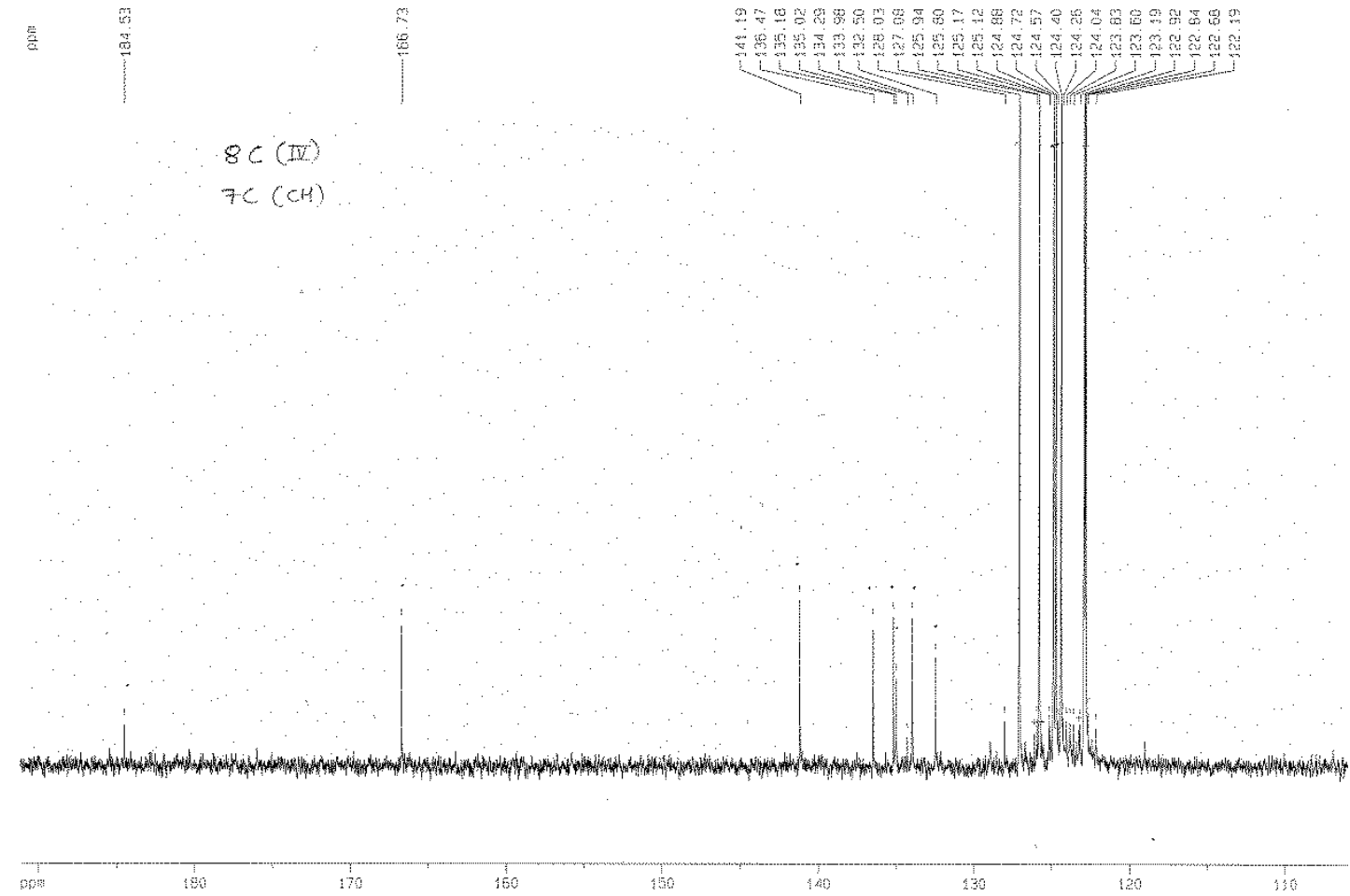

\section{$D E-M S$}

||193.206.72.116ldl...IPasinilpasini52

18/07/2018 11:36:27

GC 17

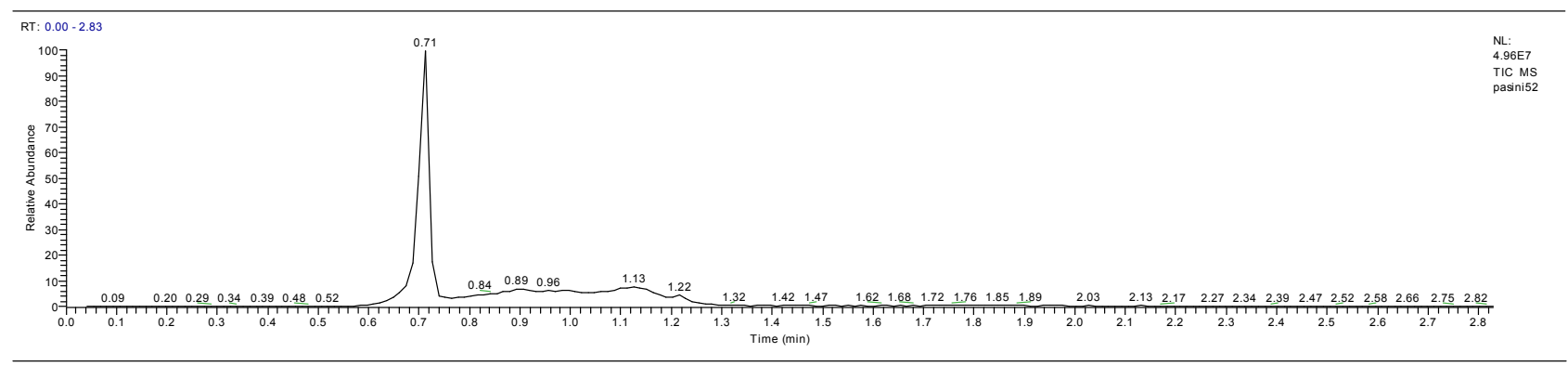

pasini52 \#53 RT: 0.71 AV: 1 NL: 9.53E6
T: + c cull ms $[50.00-1000.00]$

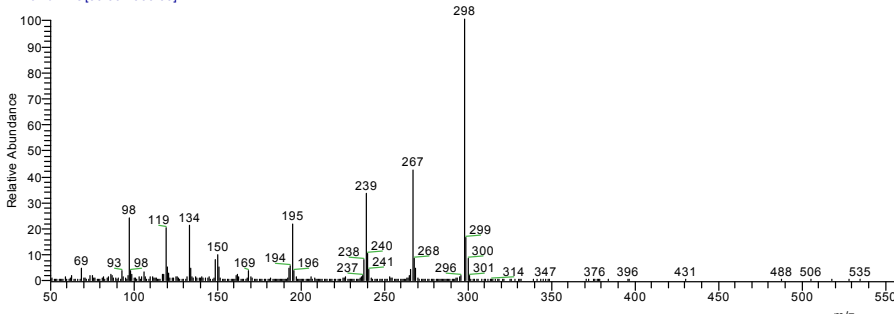


Compound 13

${ }^{1} \mathrm{HNMR}\left(200 \mathrm{MHz}, \mathrm{CDCl}_{3}\right)$<smiles>COC(=O)c1cc2c3ccccc3sc2c2sccc12</smiles>

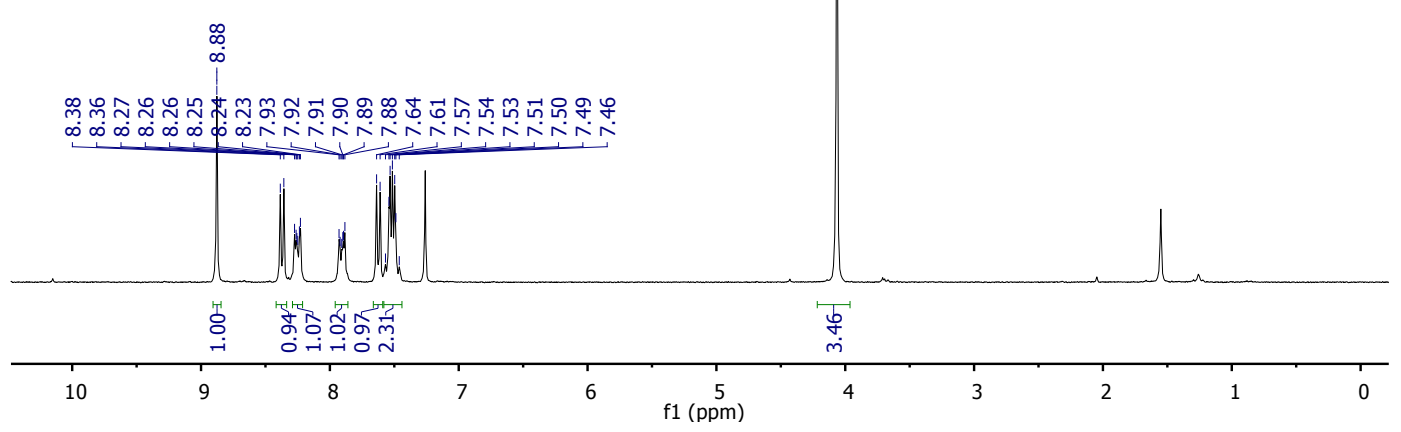

${ }^{13} \mathrm{C} \mathrm{NMR}\left(75 \mathrm{MHz}, \mathrm{CDCl}_{3}\right)$

E

듬 중요

ミミ以只

V

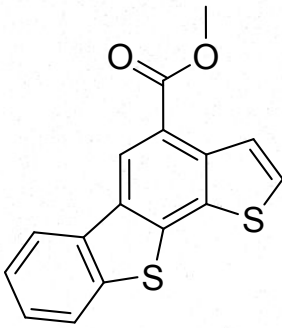


DE-MS

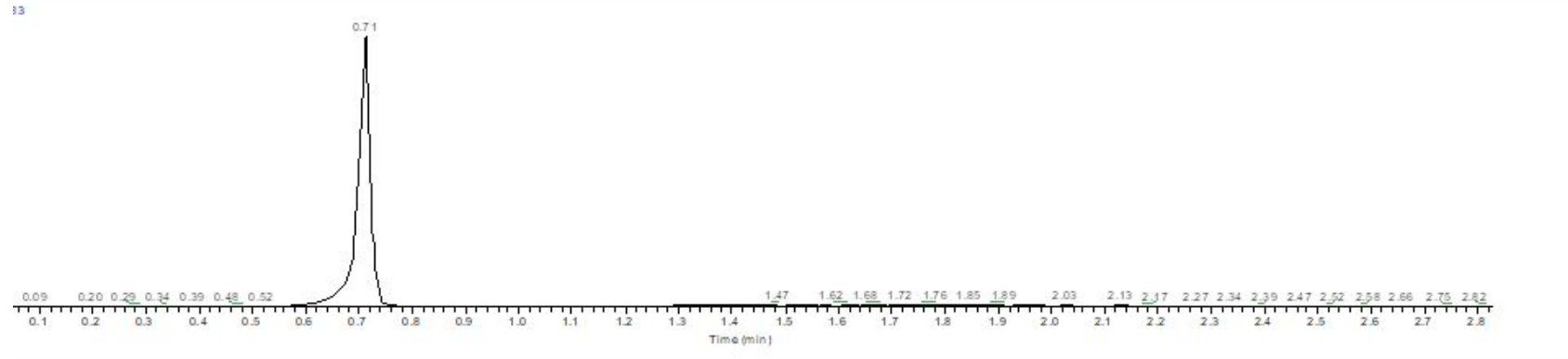

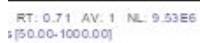

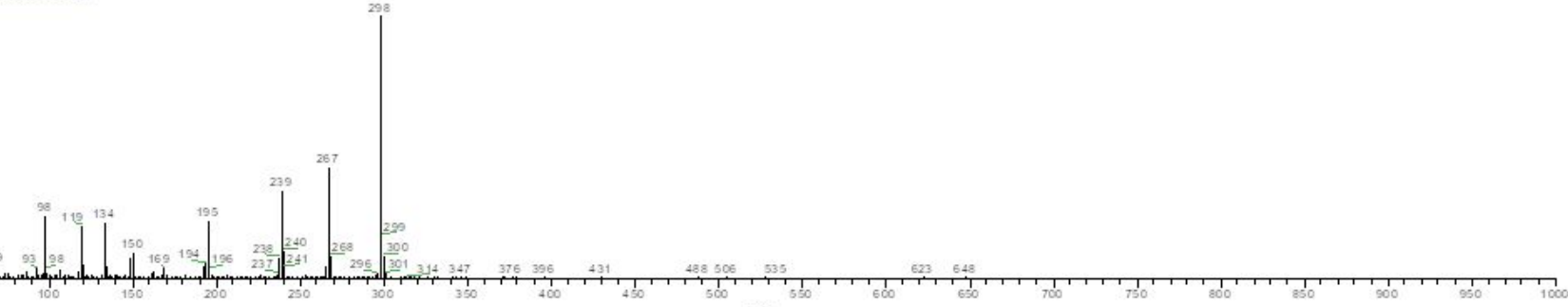


Compound 14

${ }^{1} \mathrm{HNMR}\left(300 \mathrm{MHz}, \mathrm{CDCl}_{3}\right)$<smiles>COC(=O)c1cc2c3ccccc3oc2c2sccc12</smiles>

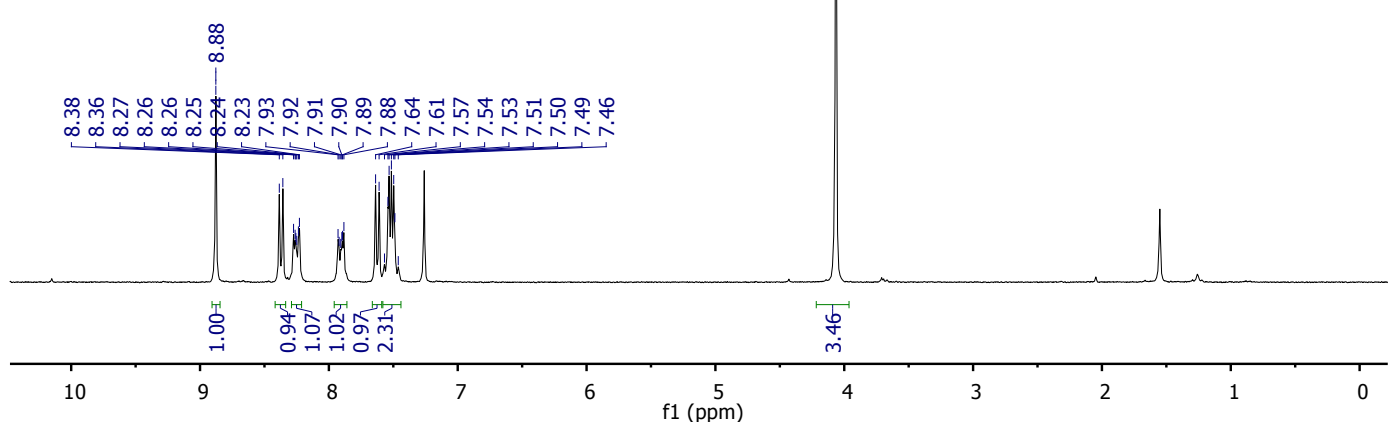

${ }^{13} \mathrm{CNMR}\left(90 \mathrm{MHz}, \mathrm{CDCl}_{3}\right)$
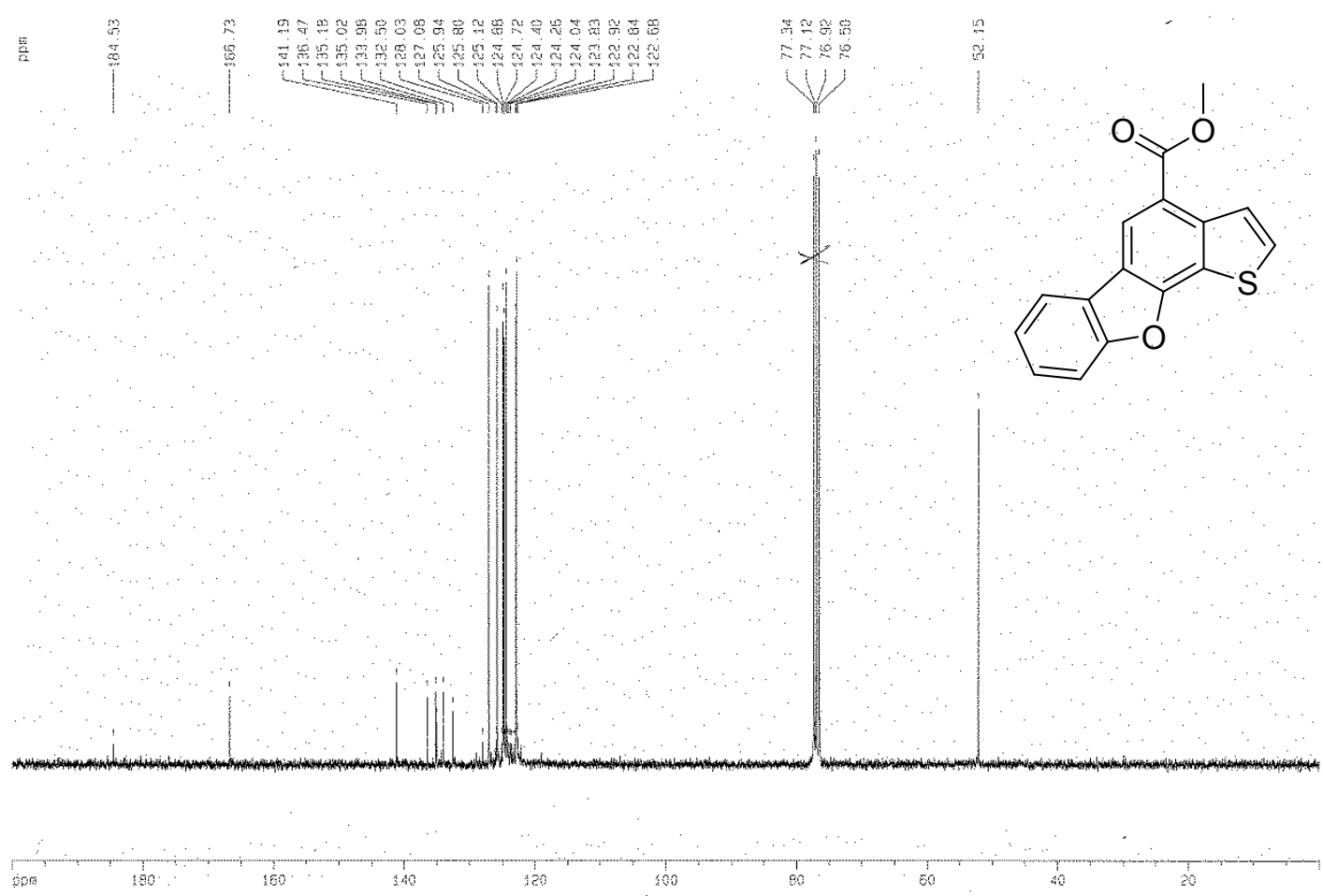


\section{Compound 15}

${ }^{1} \mathrm{HNMR}\left(300 \mathrm{MHz}, \mathrm{CDCl}_{3}\right)$

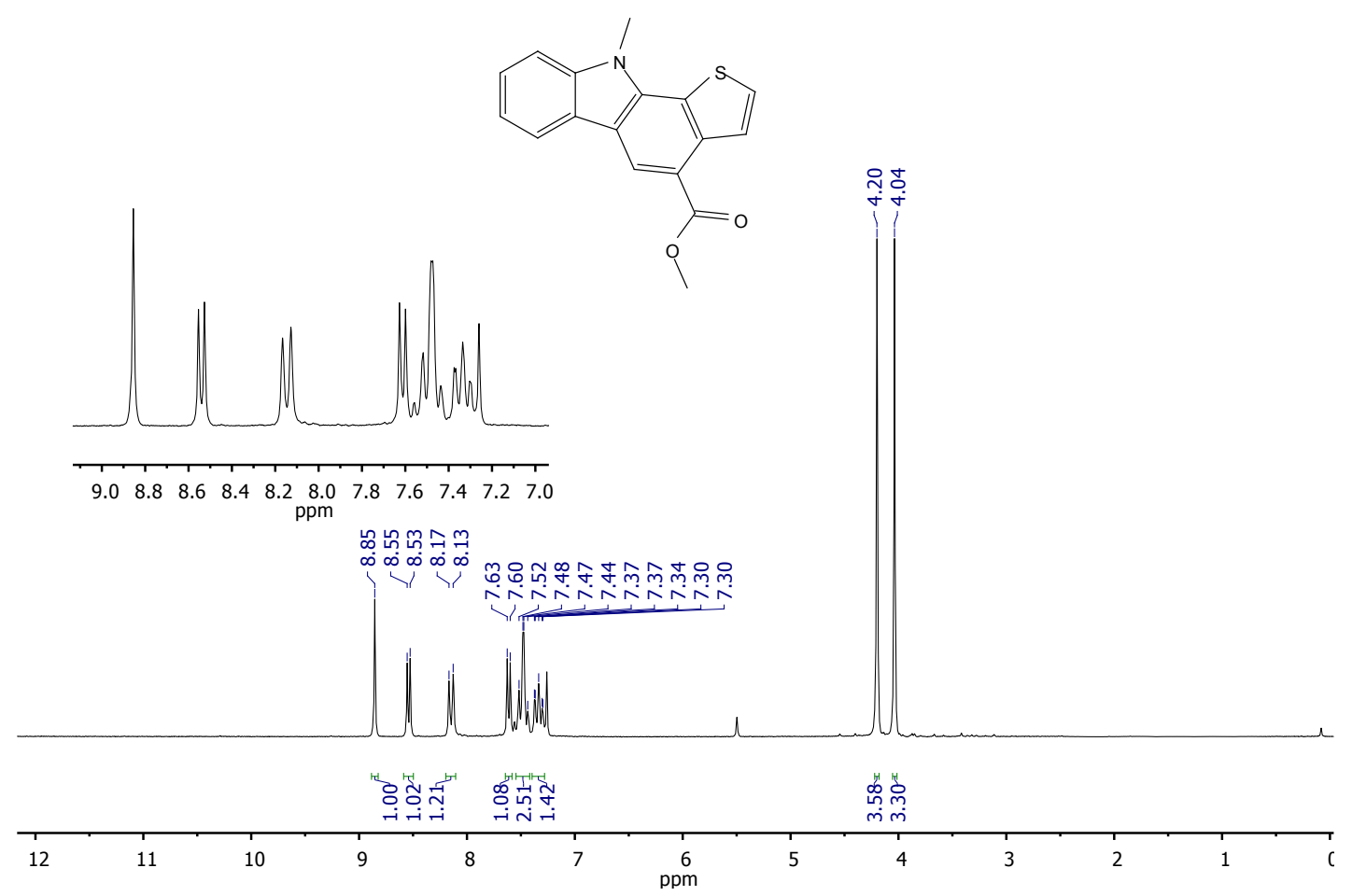

${ }^{13} \mathrm{C} \mathrm{NMR}\left(75 \mathrm{MHz}, \mathrm{CDCl}_{3}\right)$

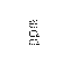

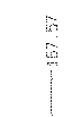

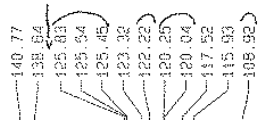

(1)

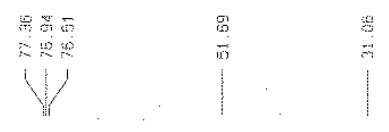

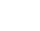



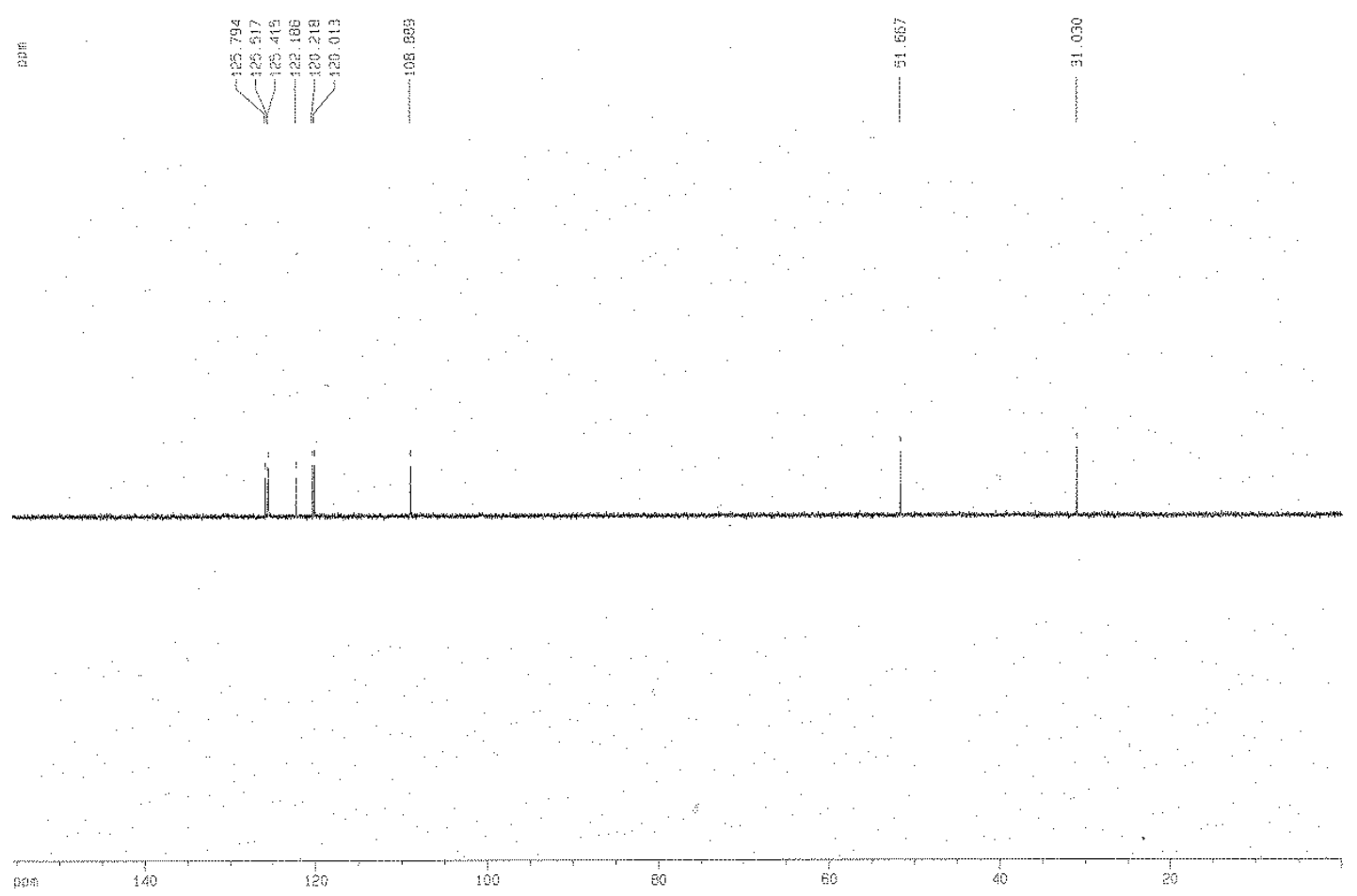

\section{ESI-MS}

D:LAVORICGS_2018IUNIPVIPasinilpasini14 GC47 M2 in $\mathrm{CH} 2 \overline{\mathrm{Cl}} 2 / \mathrm{MeOH}$

9/25/2018 11:14:14 AM

GC47 M2

pasini14 \#1 RT: 0.00 AV: $1 \quad$ NL: $4.52 E 4$
T: TMS + c ESI Full ms [50.00-2000.00]

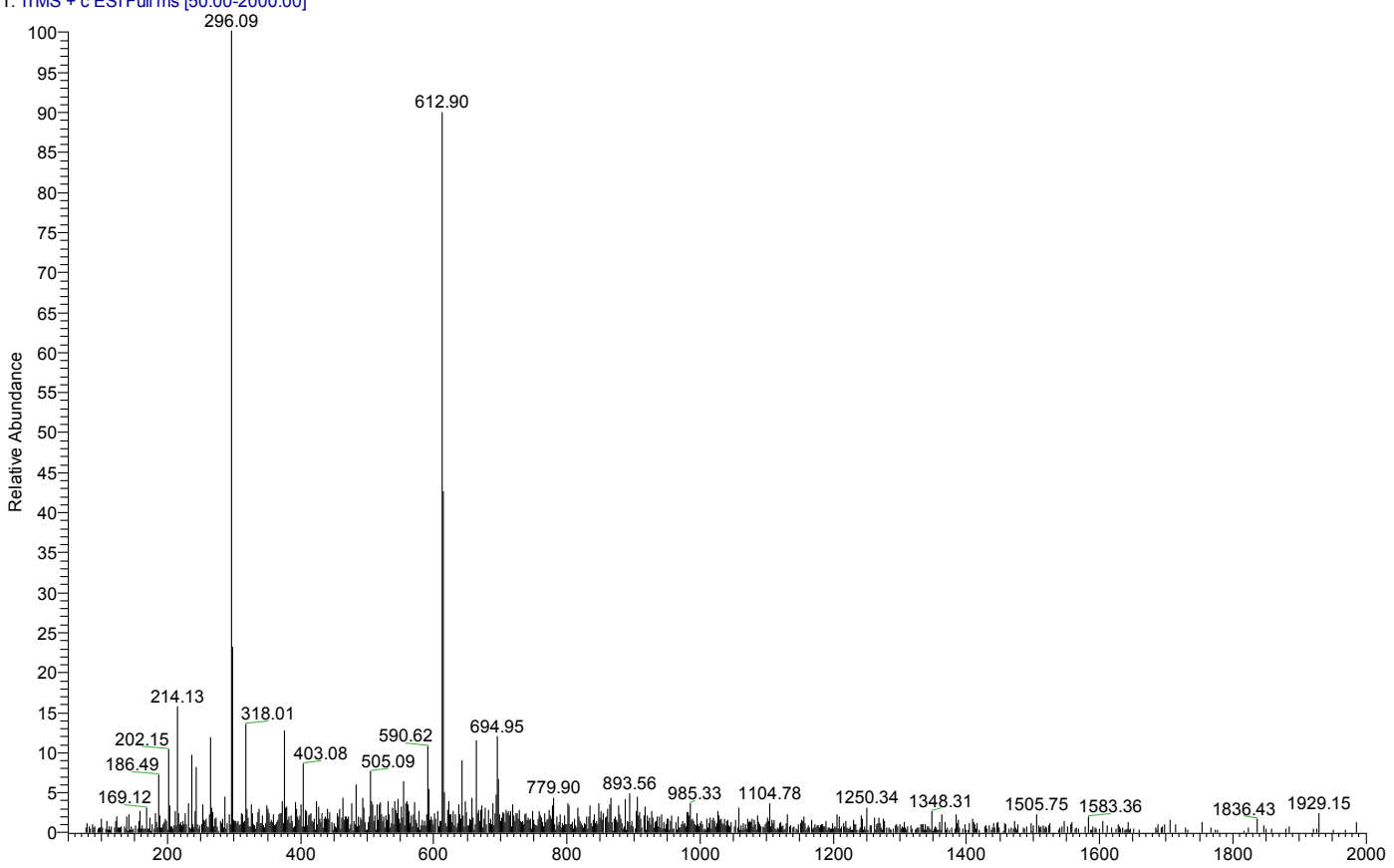




\section{Compound 16}

${ }^{1} \mathrm{HNMR}\left(300 \mathrm{MHz}, \mathrm{CDCl}_{3}\right)$

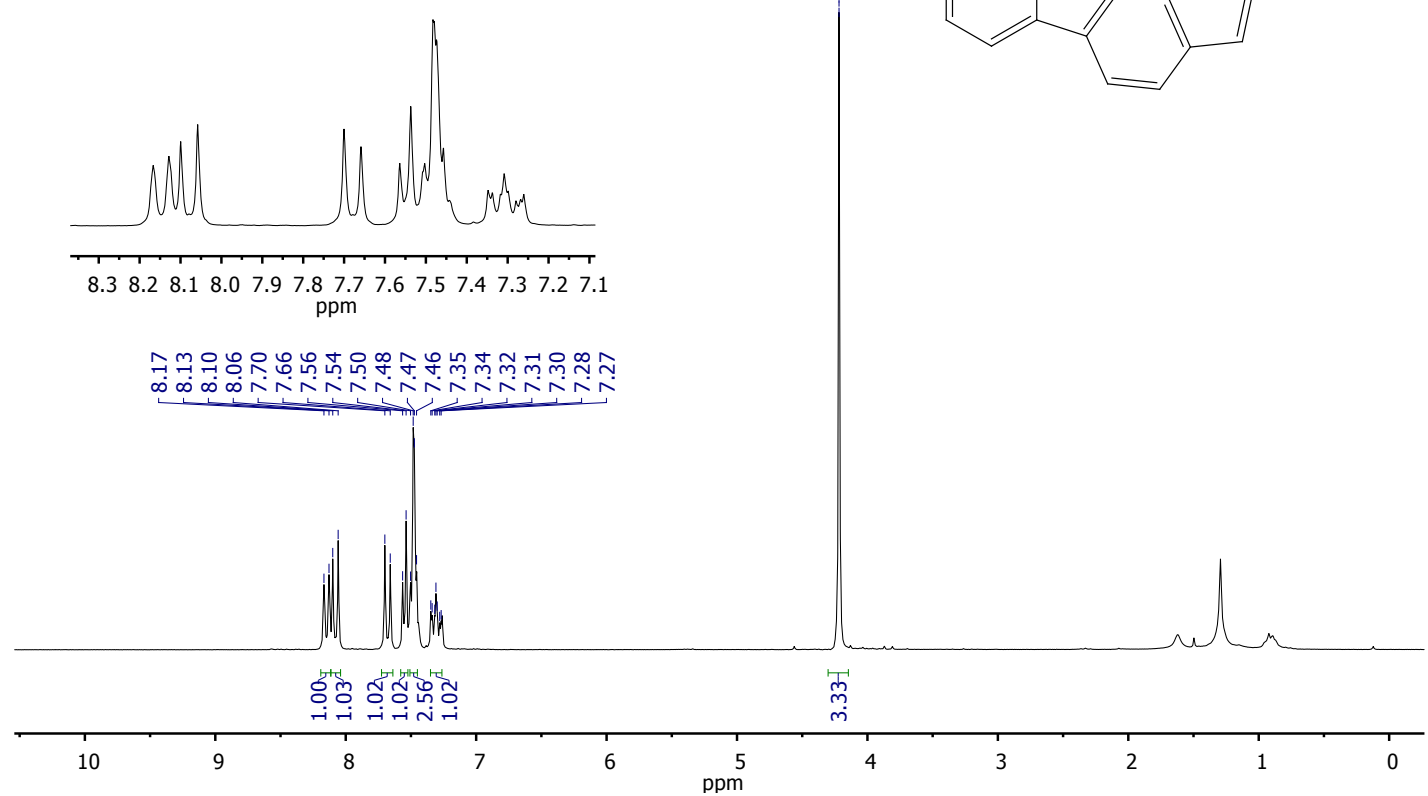

${ }^{13} \mathrm{C} \mathrm{NMR}\left(75 \mathrm{MHz}, \mathrm{CDCl}_{3}\right)$

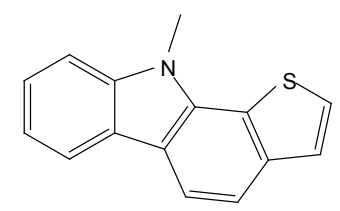<smiles>Cn1c2ccccc2c2ccc3ccsc3c21</smiles>

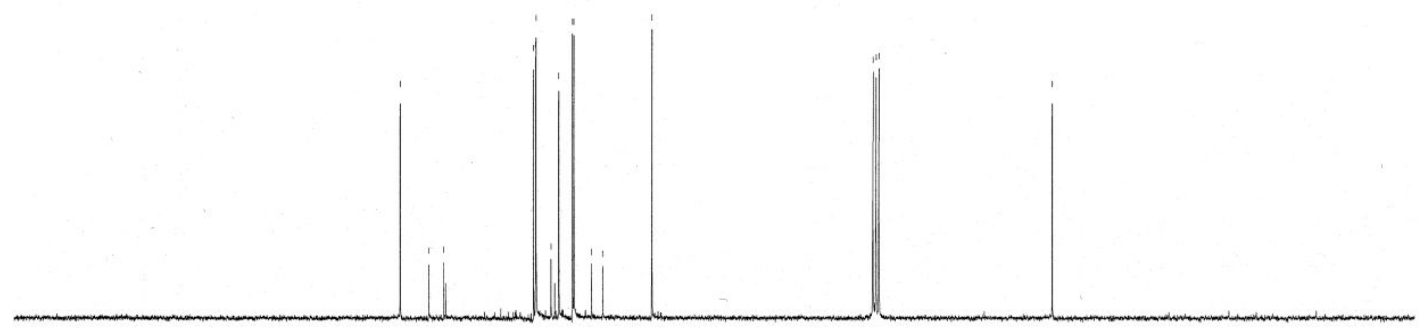

$180+160+140,120$

100

80

60

40

20 


\section{ESI-MS}

D:ILAVORICGS_2018IUNIPVIPasinilpasini13

pasini13\#1 RT: 0.00 AV: 1 NL: 7.12E4

T: TTMS + c ESI Full ms [50.00-1500.00]

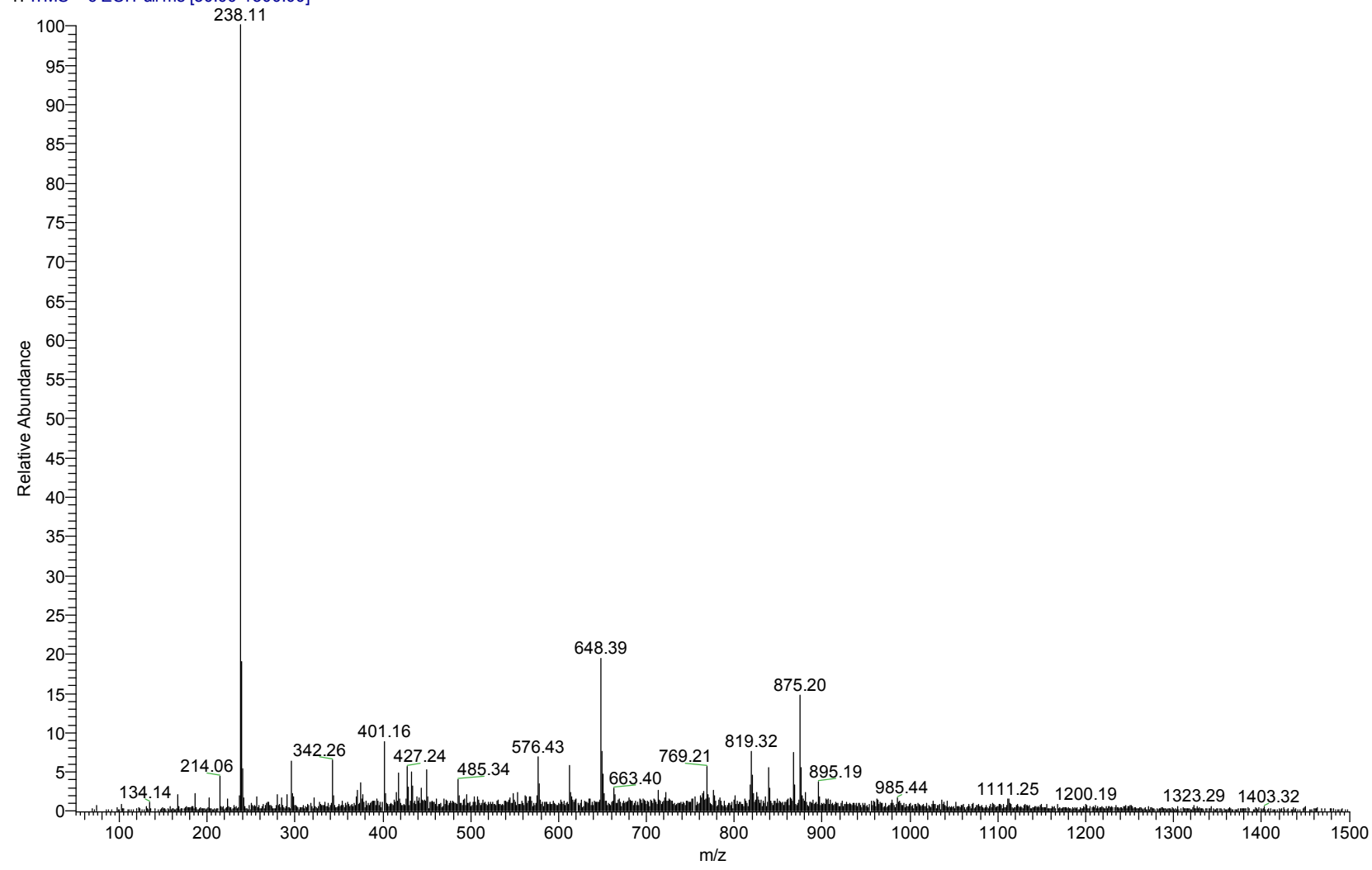


Compound 17

${ }^{l} \mathrm{HNMR}\left(300 \mathrm{MHz}, \mathrm{CDCl}_{3}\right)$

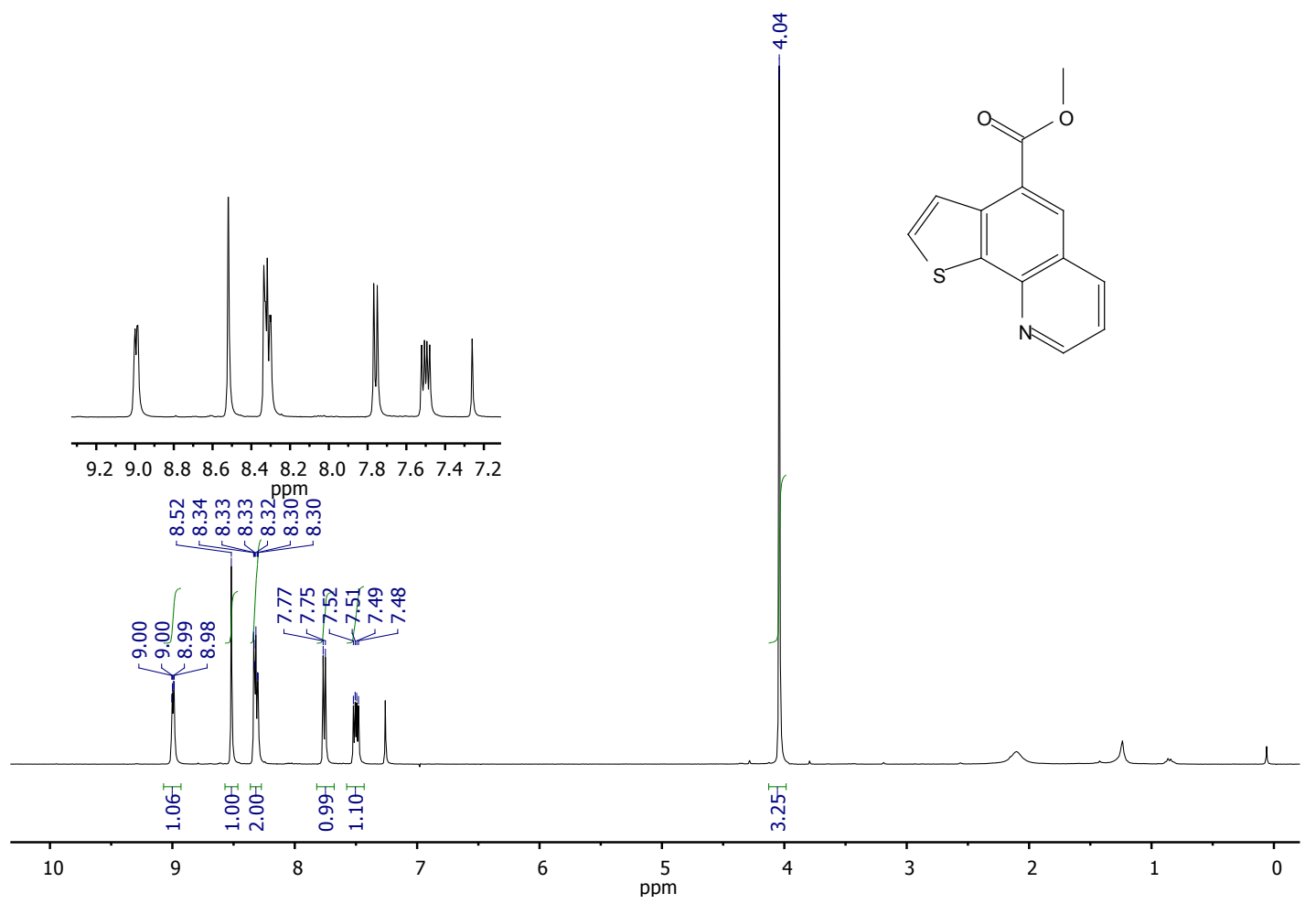

${ }^{13} \mathrm{C} \mathrm{NMR}\left(90 \mathrm{MHz}, \mathrm{CDCl}_{3}\right)$<smiles></smiles>

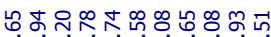

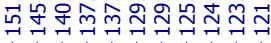

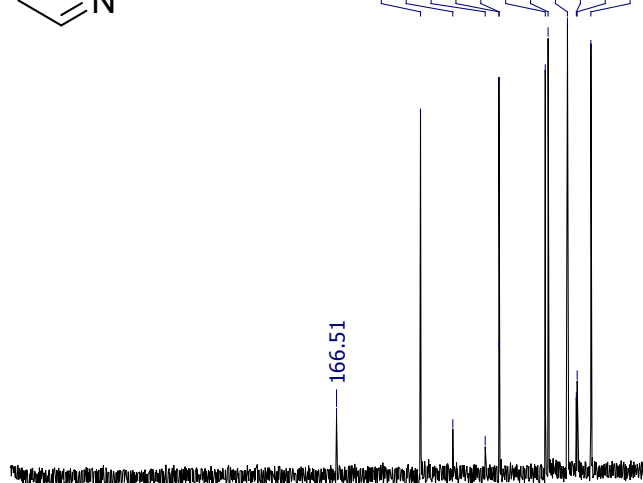

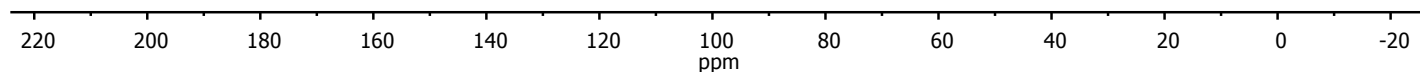


ESI-MS

pasini03\#21 RT: $0.16 \quad \mathrm{AV}: 1 \quad \mathrm{NL}: 4.05 \mathrm{E}$

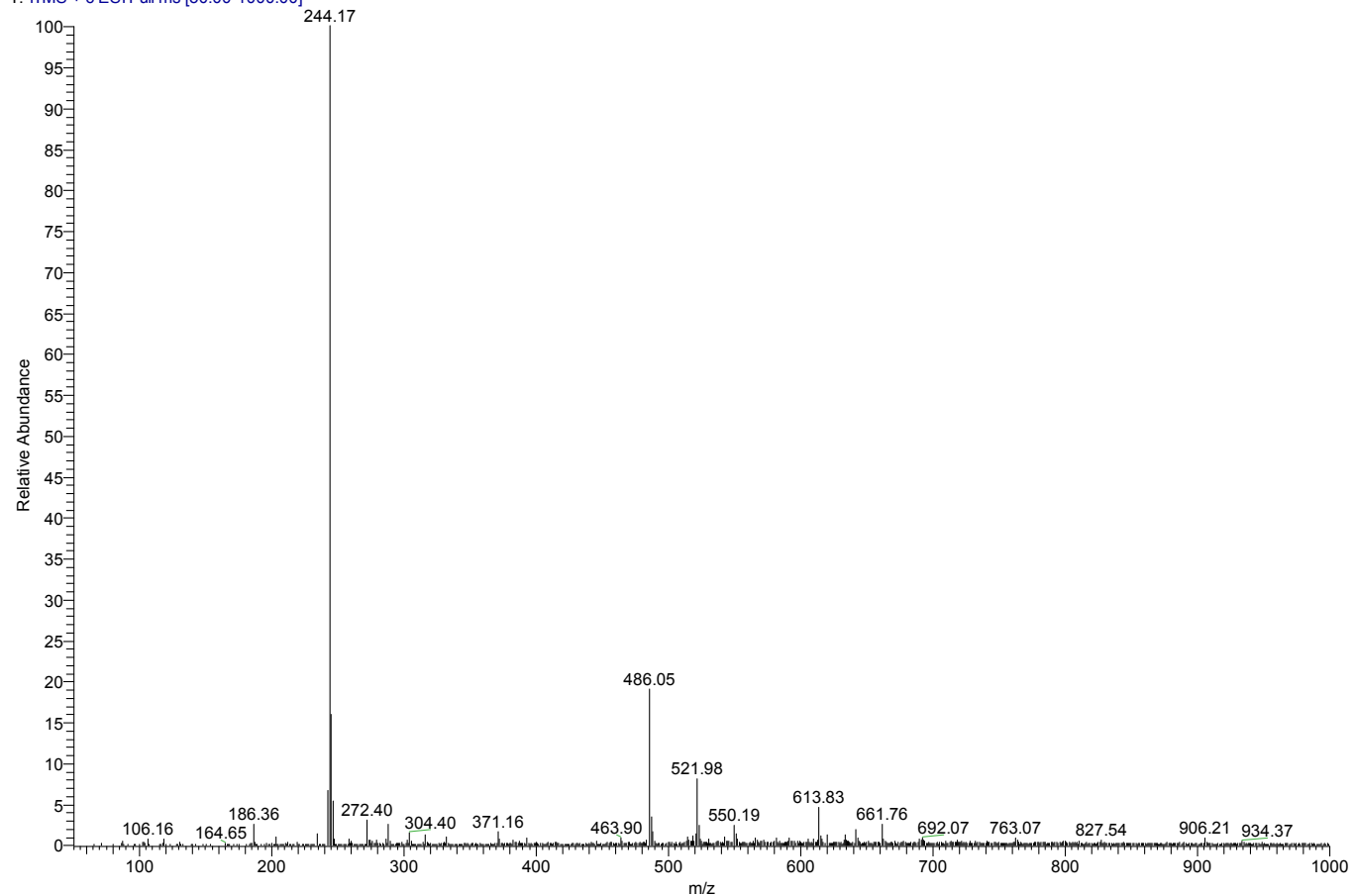




\section{Compound 18}

${ }^{1} \mathrm{H} \mathrm{NMR}\left(300 \mathrm{MHz}, \mathrm{CDCl}_{3}\right)$

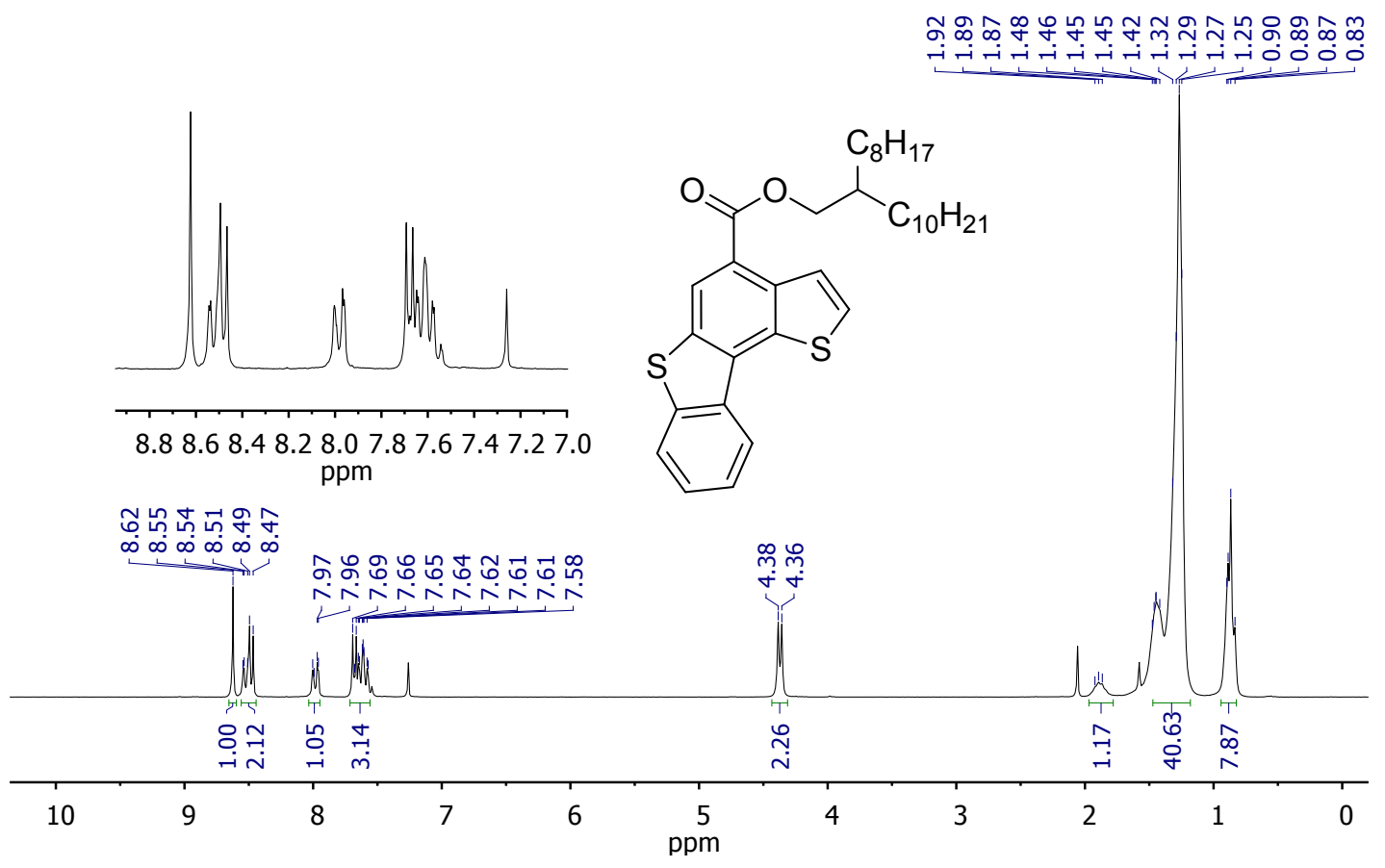

${ }^{13} \mathrm{CNMR}\left(75 \mathrm{MHz}, \mathrm{CDCl}_{3}\right)$

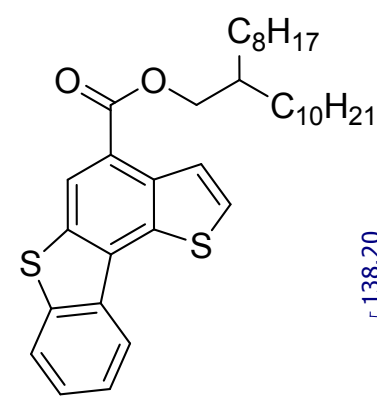

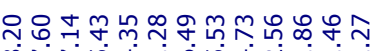

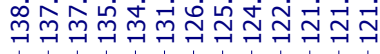

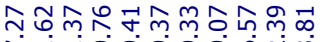

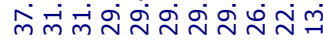

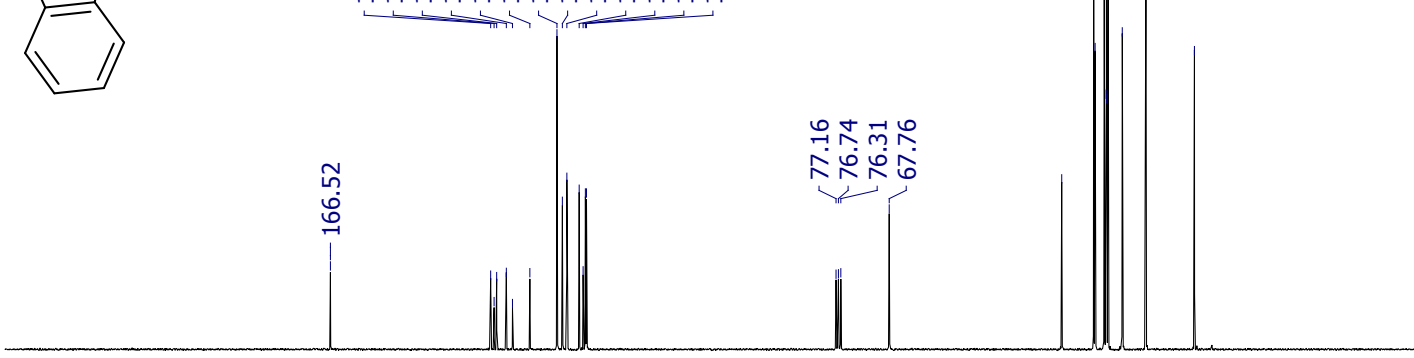

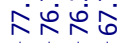

\begin{tabular}{|c|c|c|c|c|c|c|c|c|c|c|c|c|}
\hline 220 & 200 & 180 & 160 & 140 & 120 & $\begin{array}{l}100 \\
\mathrm{ppm}\end{array}$ & 80 & 60 & 40 & 20 & 0 & -20 \\
\hline
\end{tabular}


$D E-M S$

D:LAVORICGS_2016IUNIPVIPasinipasini0

10/10/2017 4:56:56 PM

AN456

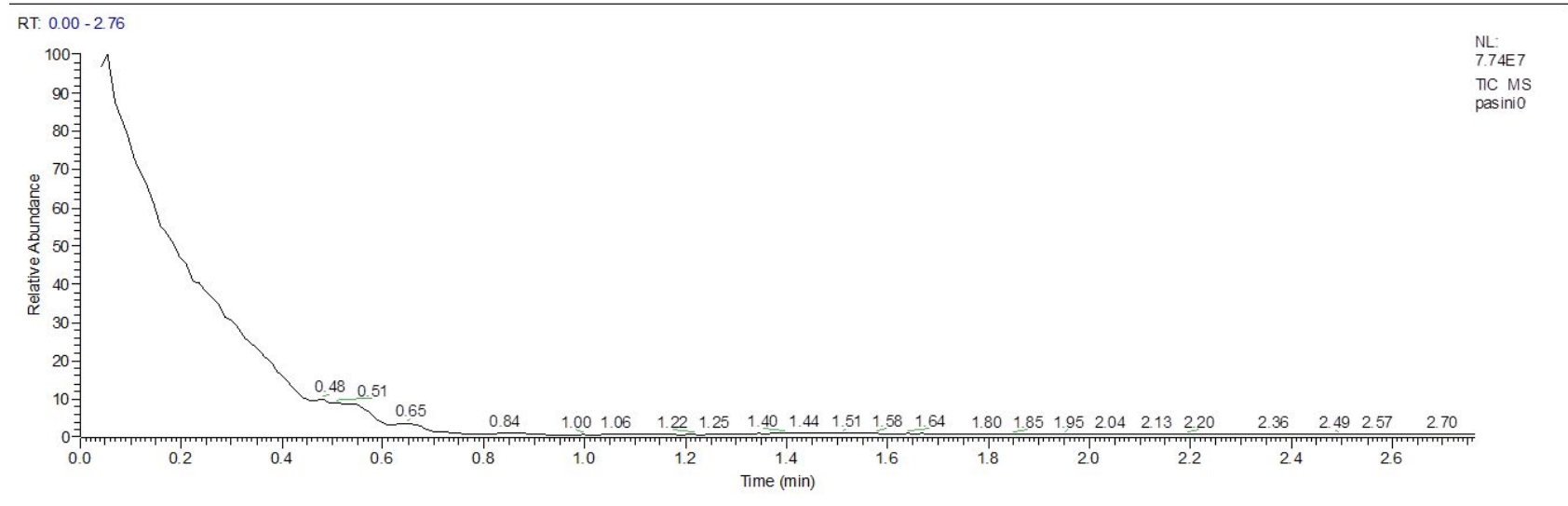

pasinio \#3 RT: 0.07 AV: 1 SB: $1260.55-0.87,1.02-2.30$ NL: $1.24 E 7$
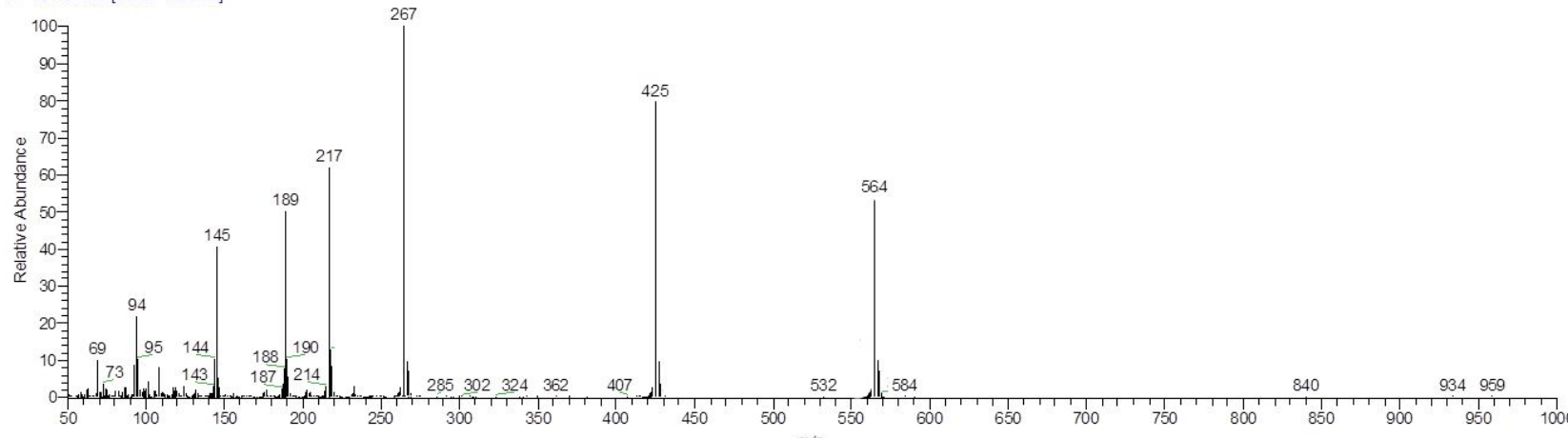
Compound 19

${ }^{1} \mathrm{HNMR}\left(300 \mathrm{MHz}, \mathrm{CDCl}_{3}\right)$

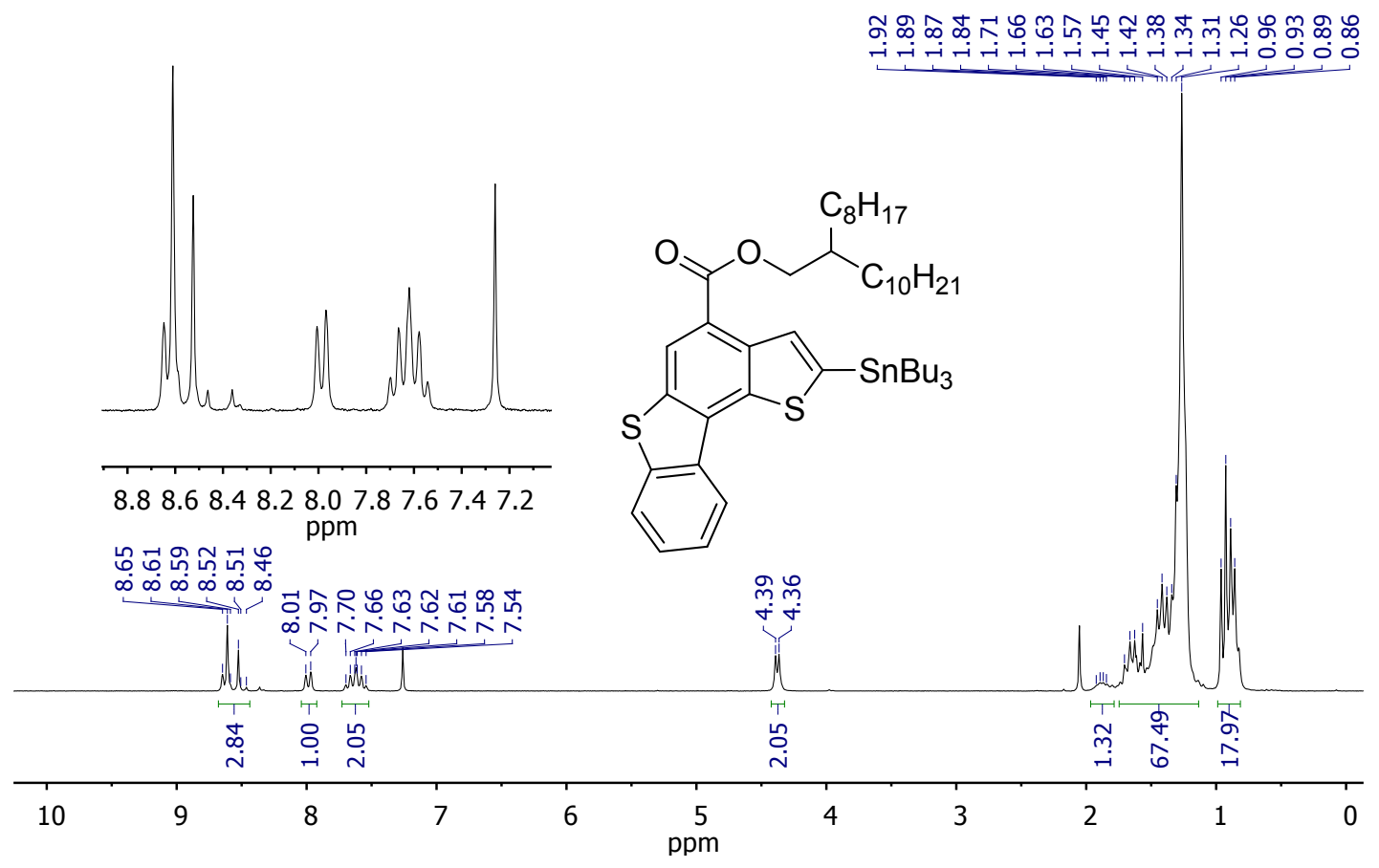

${ }^{13} \mathrm{C} \mathrm{NMR}\left(75 \mathrm{MHz}, \mathrm{CDCl}_{3}\right)$

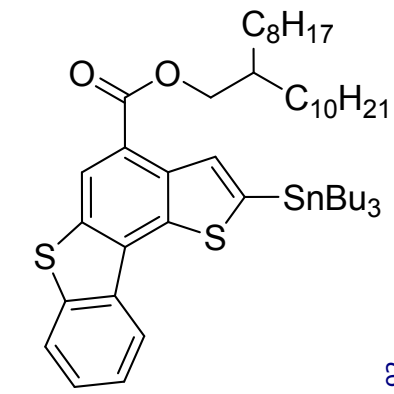

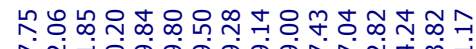

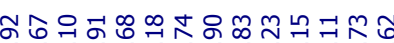

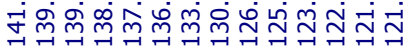

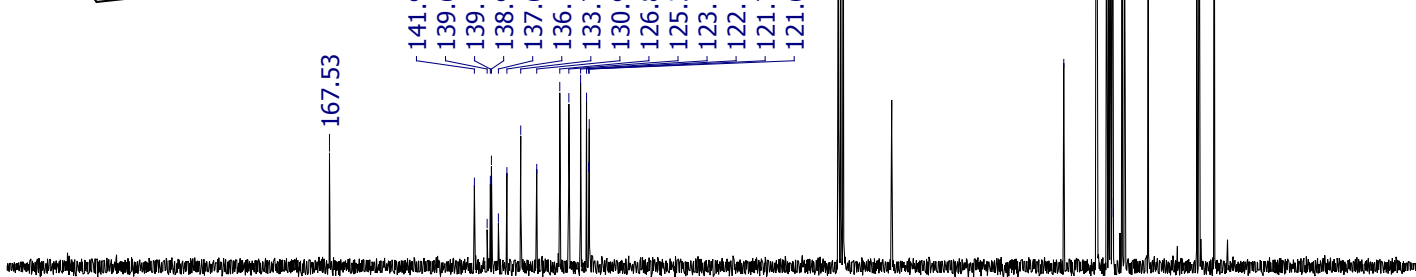

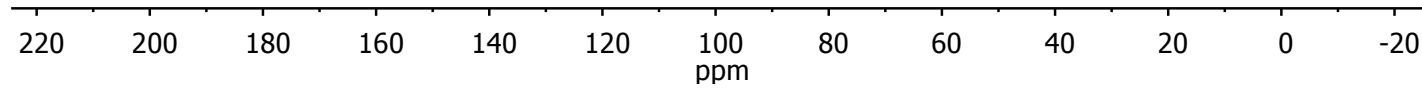




\section{Compound 20}

${ }^{l} \mathrm{HNMR}\left(300 \mathrm{MHz}, \mathrm{CDCl}_{3}\right)$

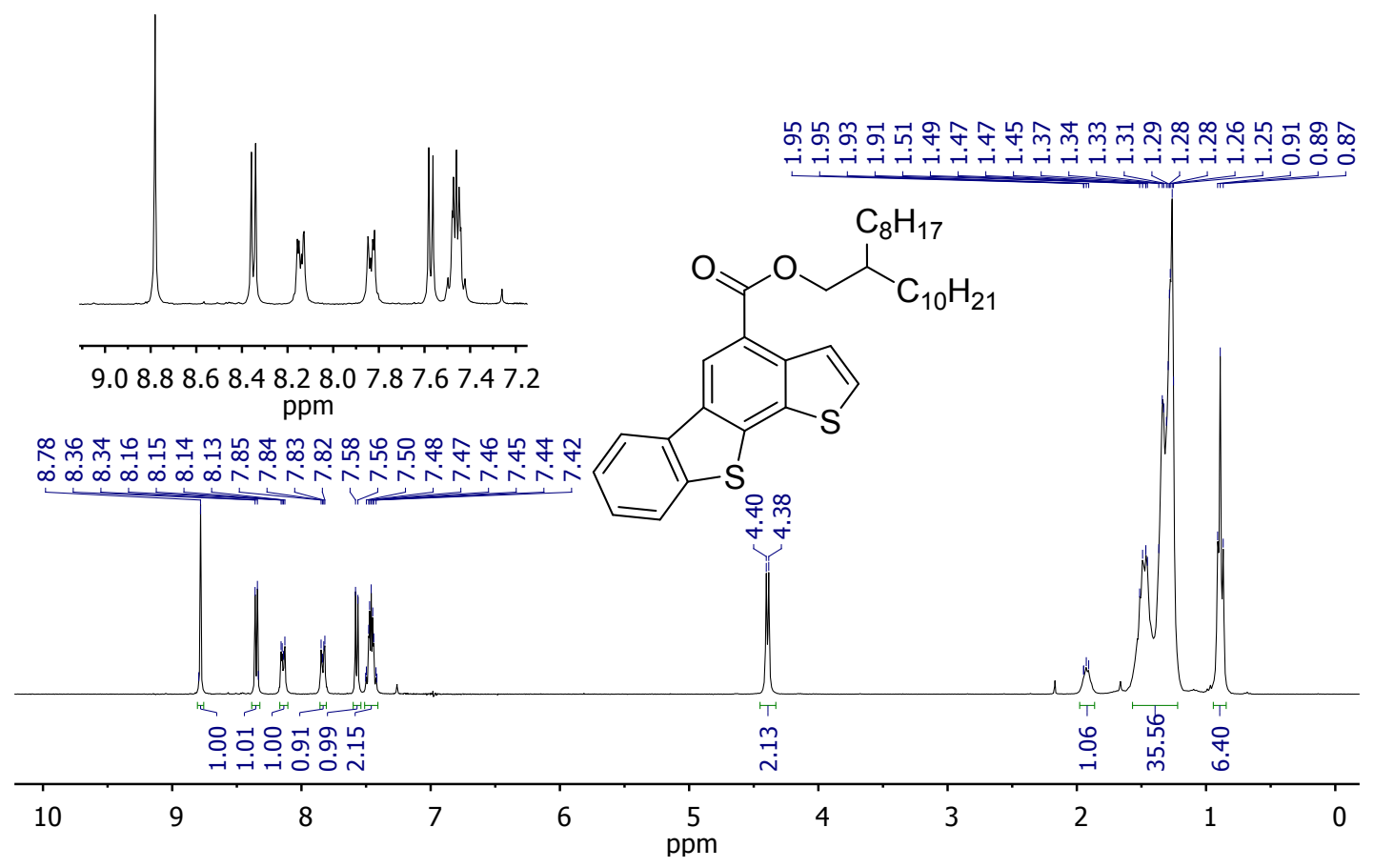

${ }^{13} \mathrm{CNMR}\left(75 \mathrm{MHz}, \mathrm{CDCl}_{3}\right)$

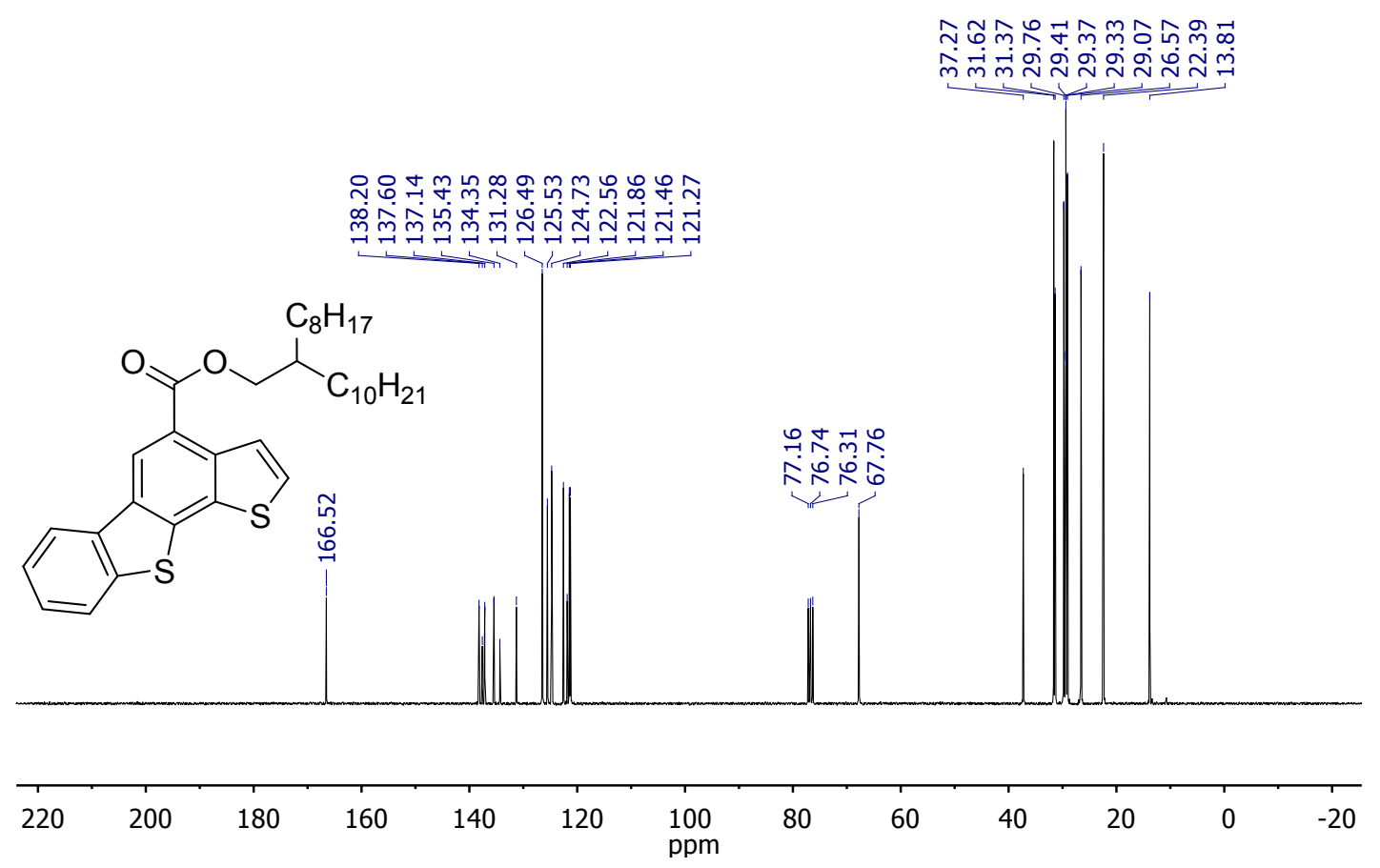




\section{$D E-M S$}

D:LAVORICGS_2016IUNIPVPasinipasini0

10/10/2017 4:56:56 PM

AN456

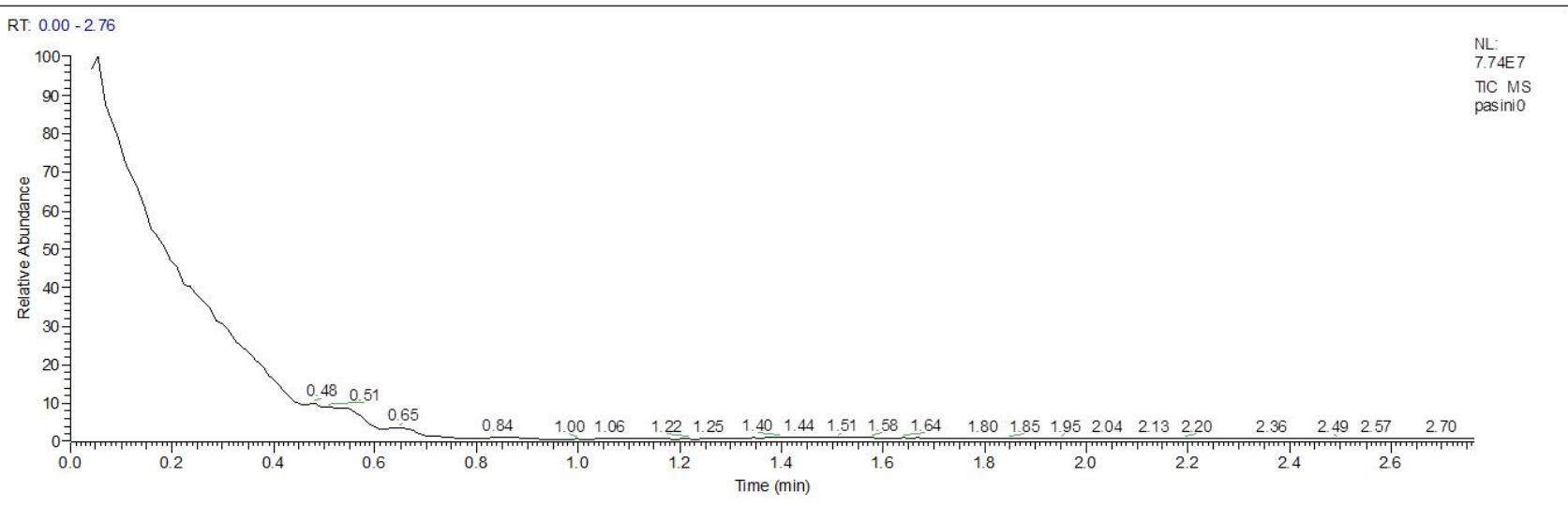

pasinio \#3 RT: 0.07 AV: 1 SB: 126 0.55-0.87, 1.02-2.30 NL: $1.24 E 7$
T: + c Full ms [50.00-1000.00]

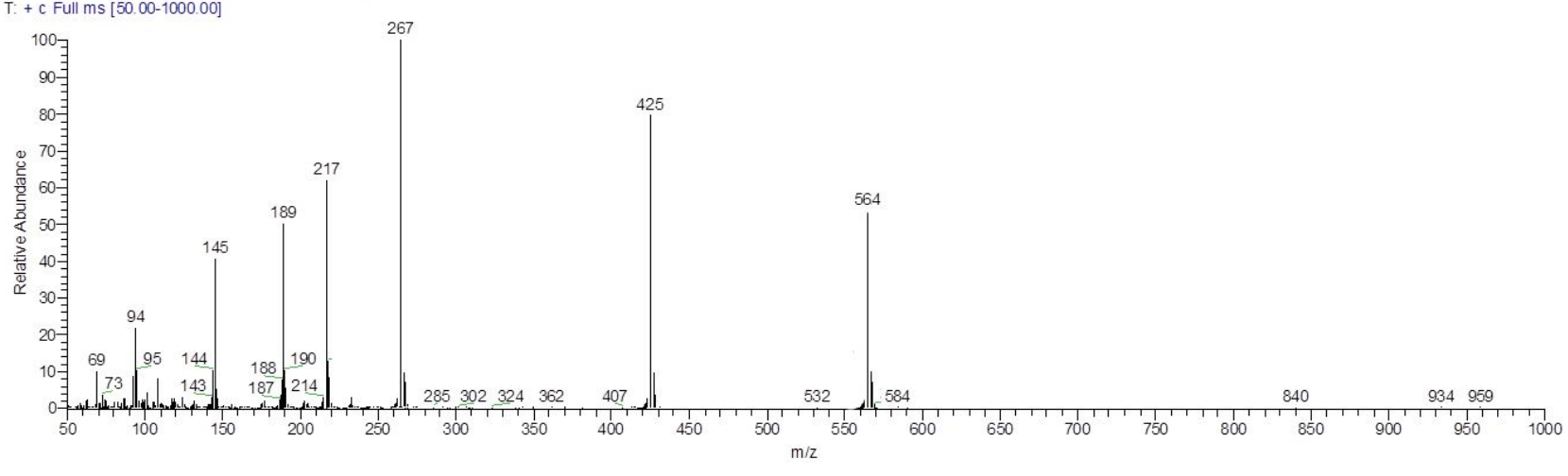


Compound 21

${ }^{1} \mathrm{HNMR}\left(300 \mathrm{MHz}, \mathrm{CDCl}_{3}\right)$

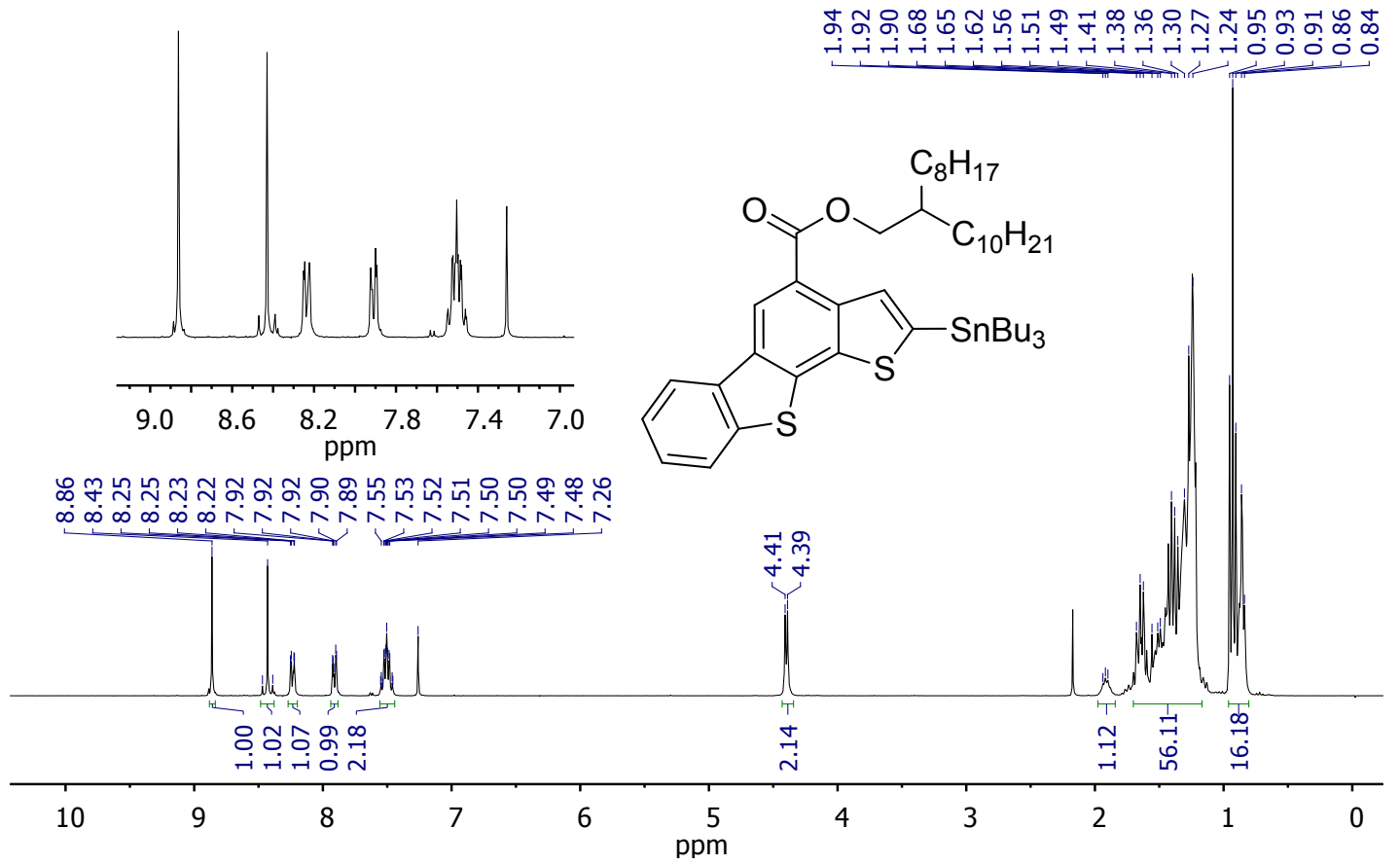

${ }^{13} \mathrm{C} \mathrm{NMR}\left(75 \mathrm{MHz}, \mathrm{CDCl}_{3}\right)$

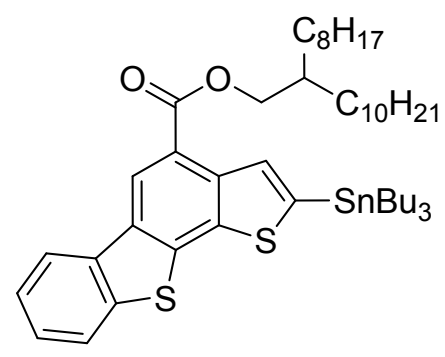

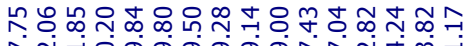

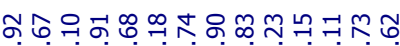

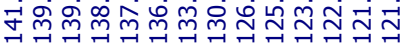

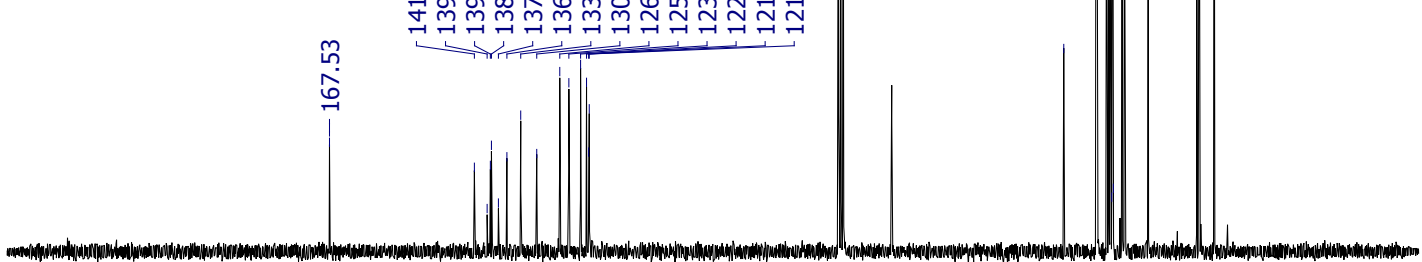

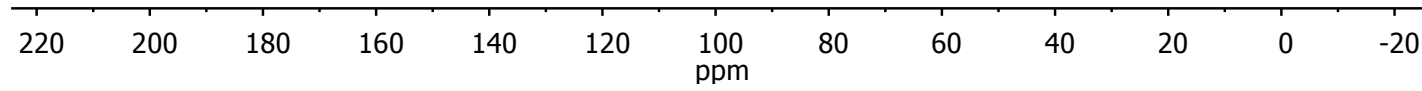


Compound 24

${ }^{1} \mathrm{H} \mathrm{NMR}\left(400 \mathrm{MHz}, \mathrm{CDCl}_{3}\right)$

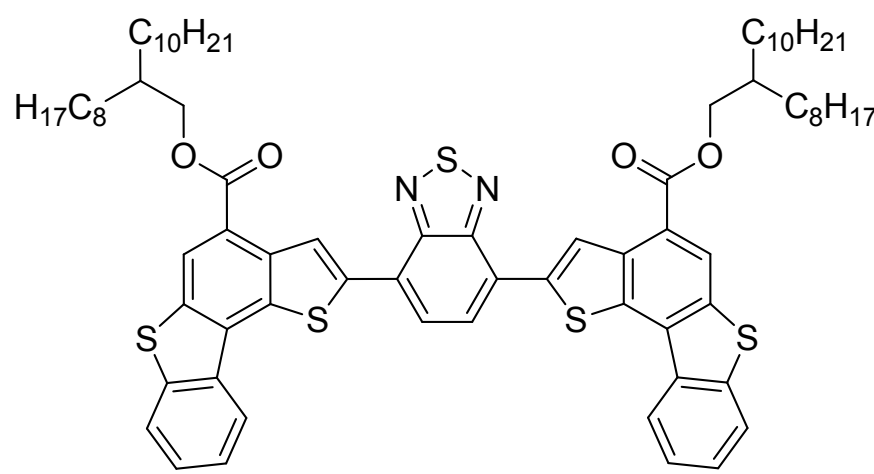

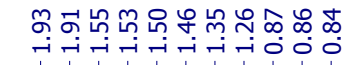

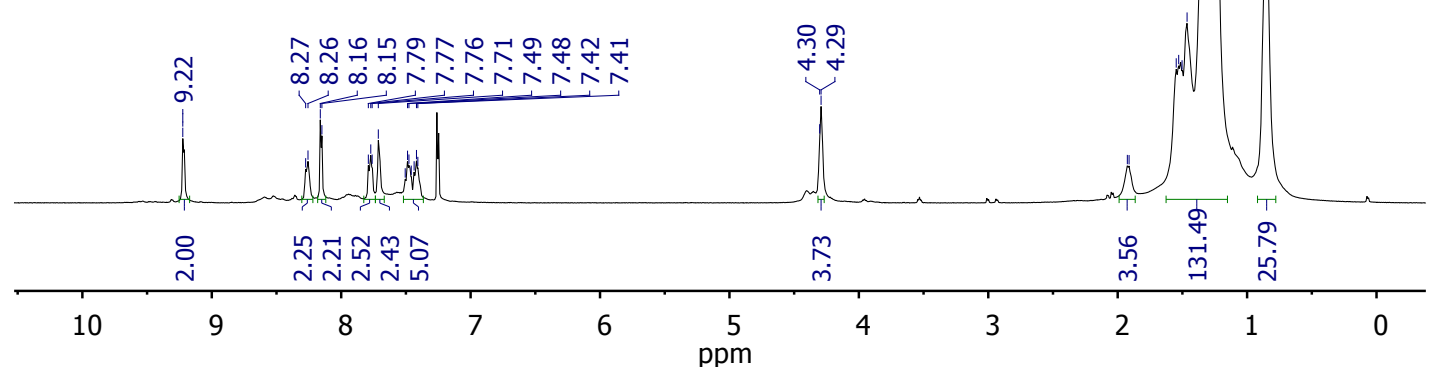

${ }^{13} \mathrm{C} \mathrm{NMR}\left(101 \mathrm{MHz}, \mathrm{CDCl}_{3}\right)$

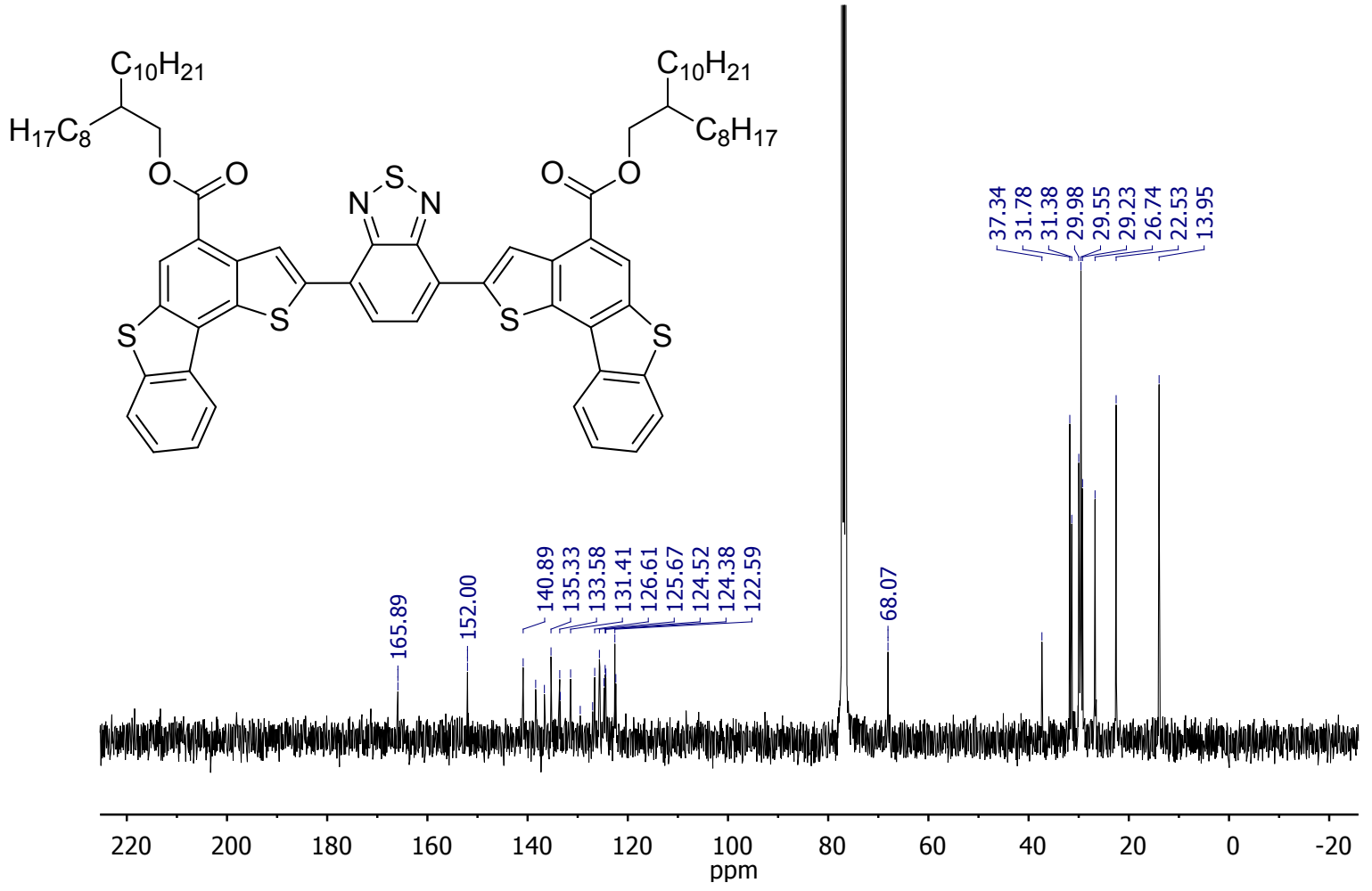




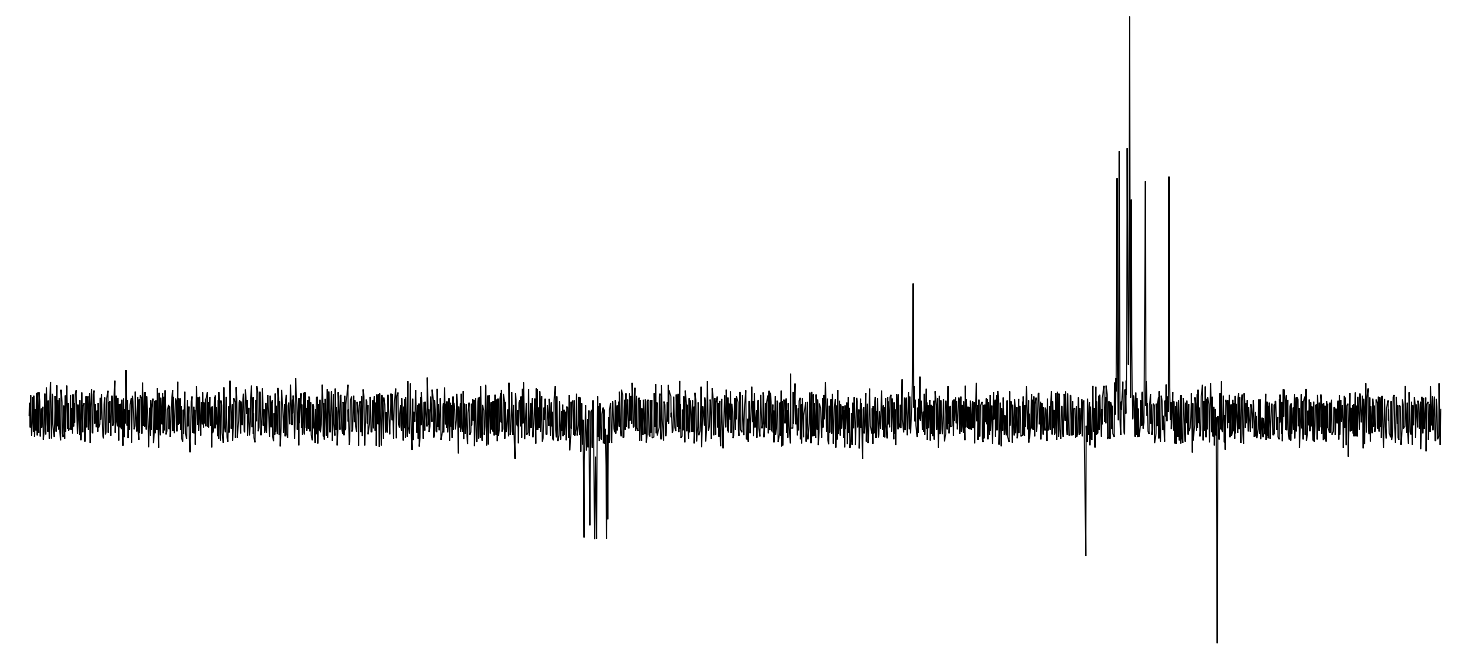

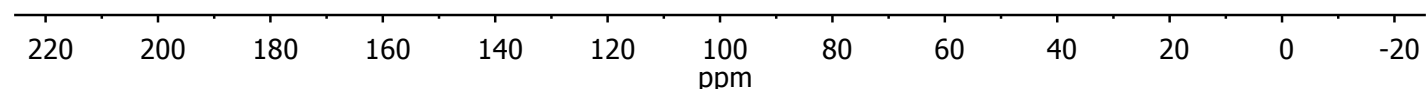

\section{$H M Q C$}

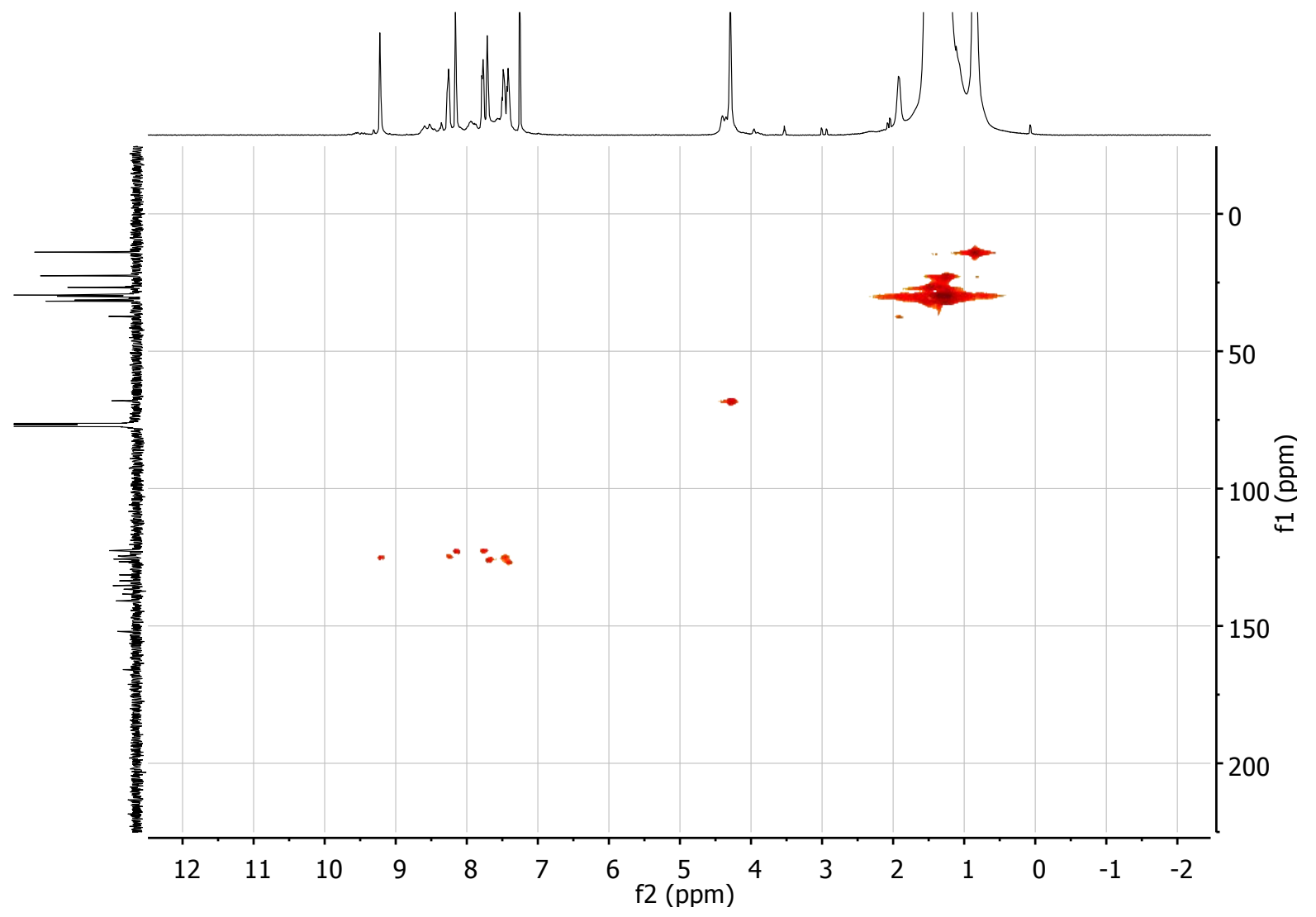




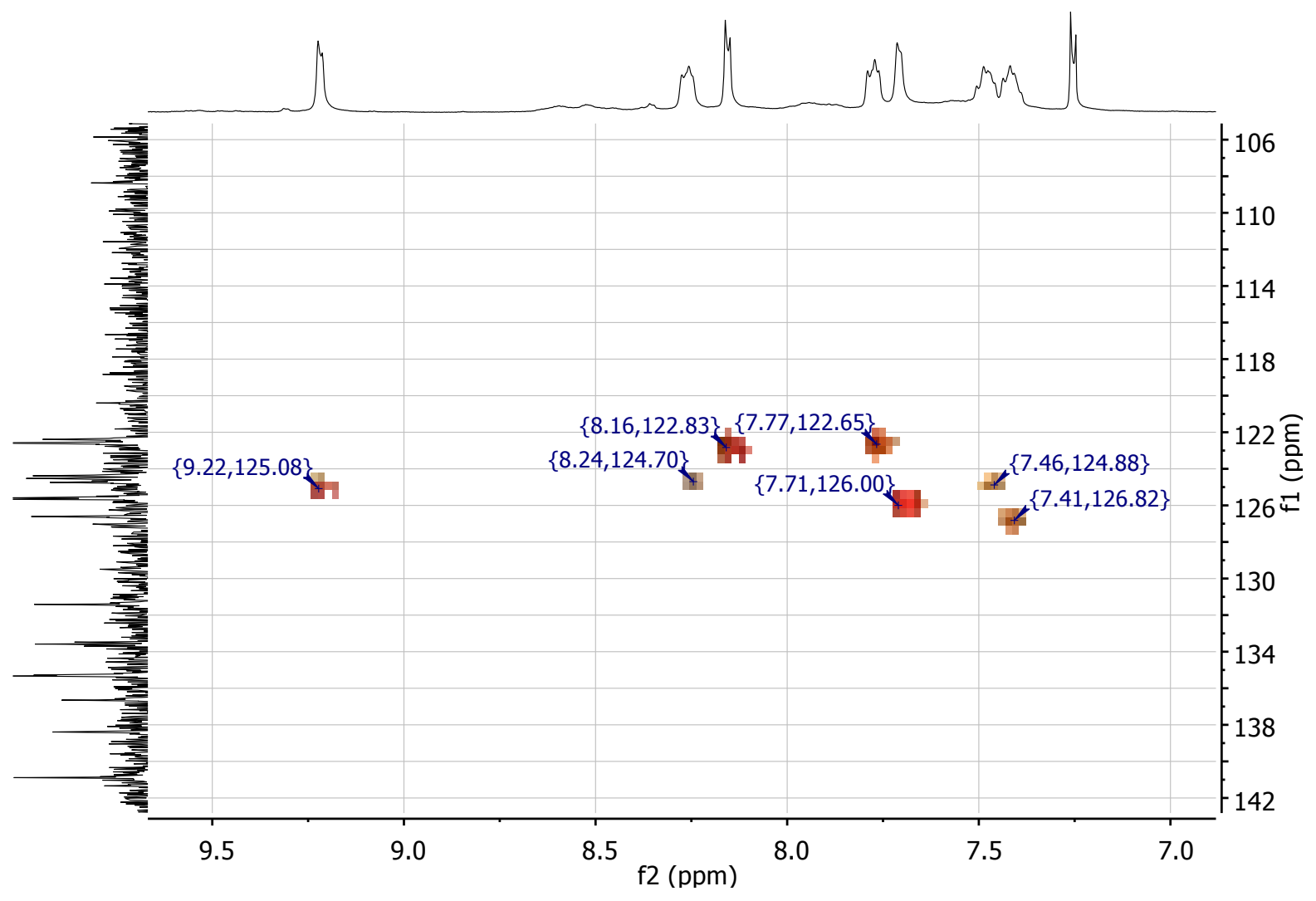

$H M B C$

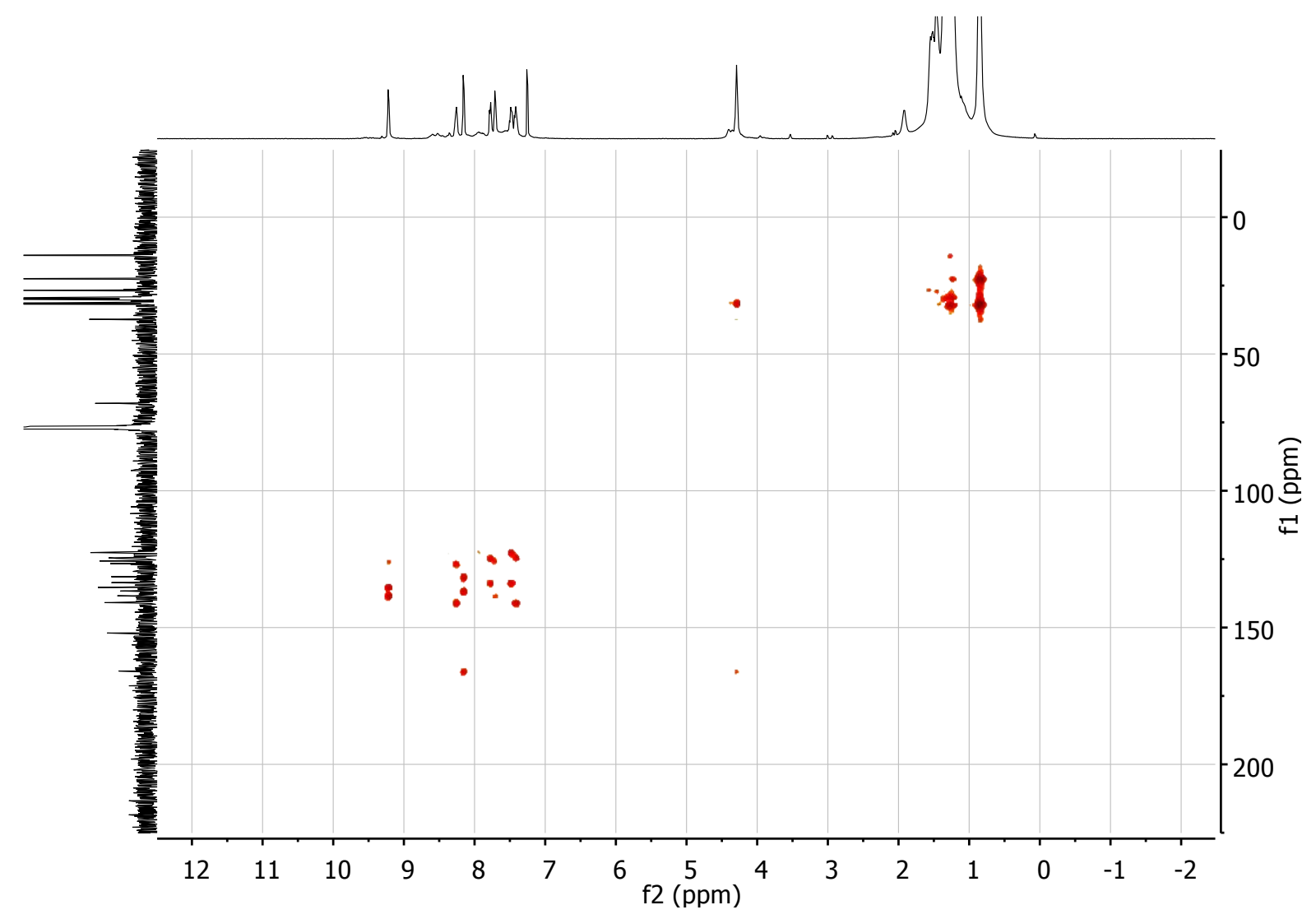




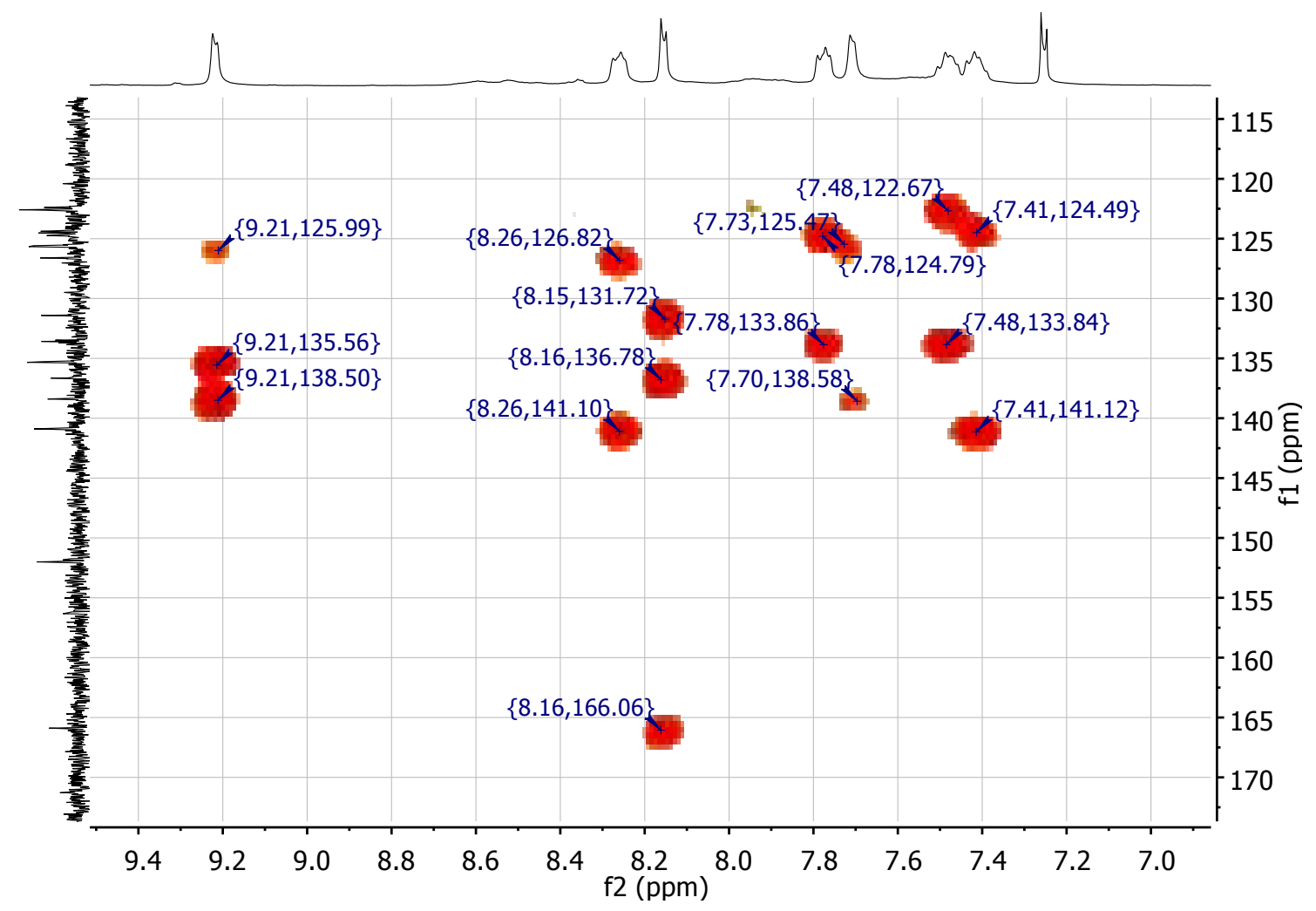

HRMS

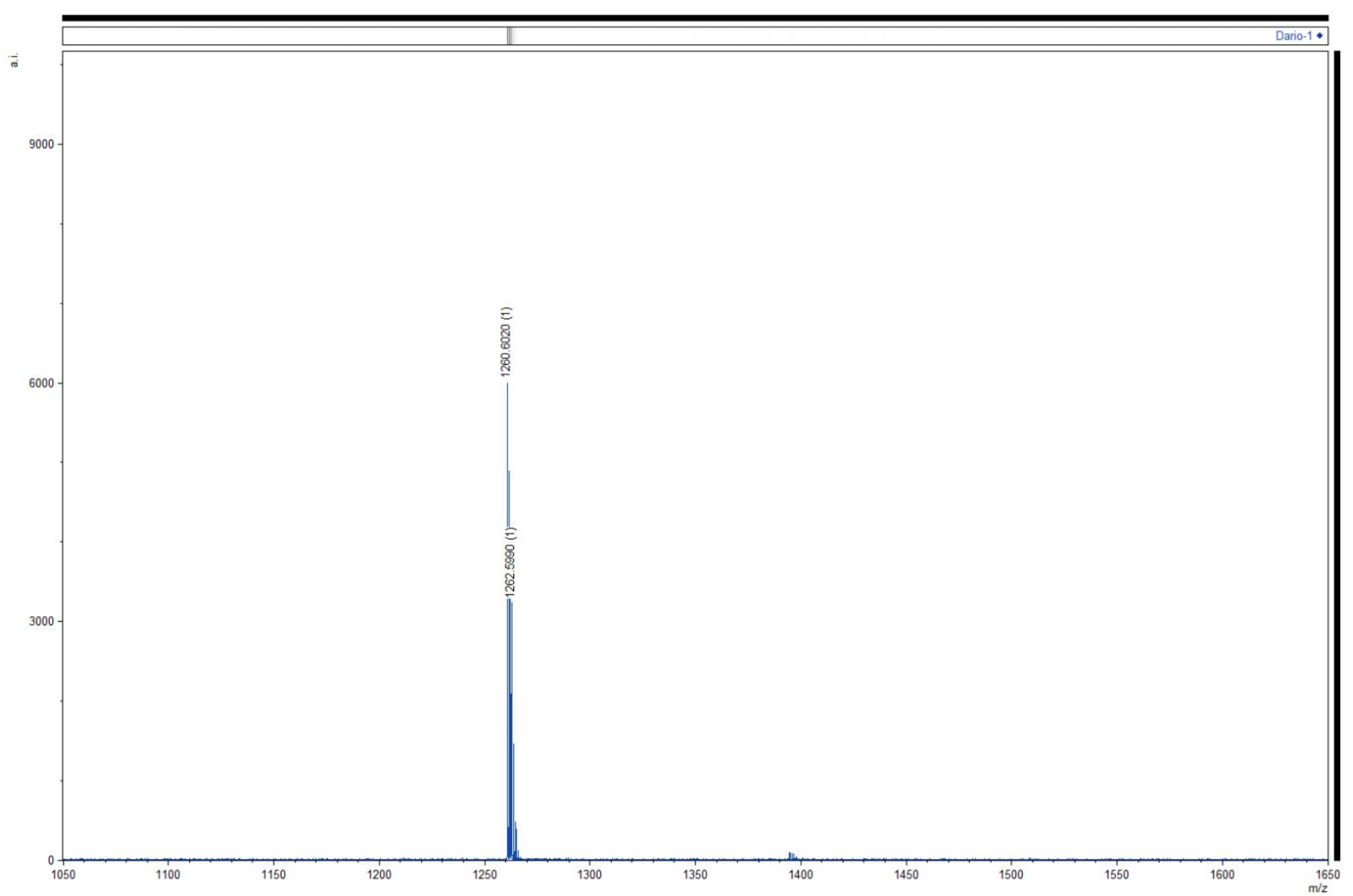




\section{Compound 25}

${ }^{1} \mathrm{HNMR}\left(400 \mathrm{MHz}, \mathrm{CDCl}_{3}\right)$
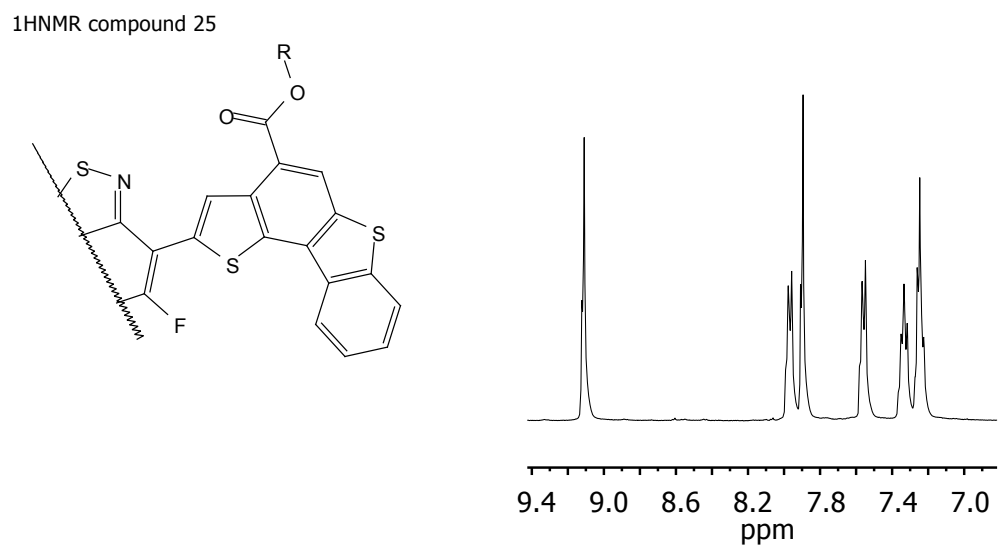

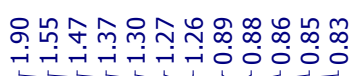

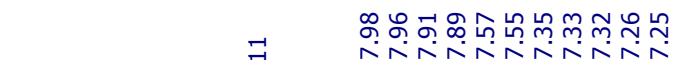

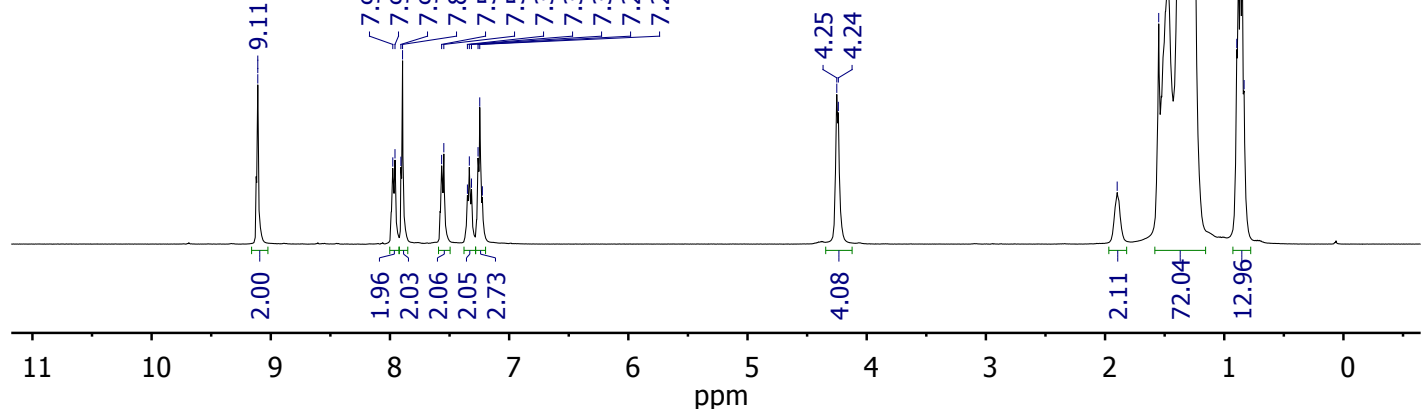

${ }^{19} \mathrm{~F} N M R$

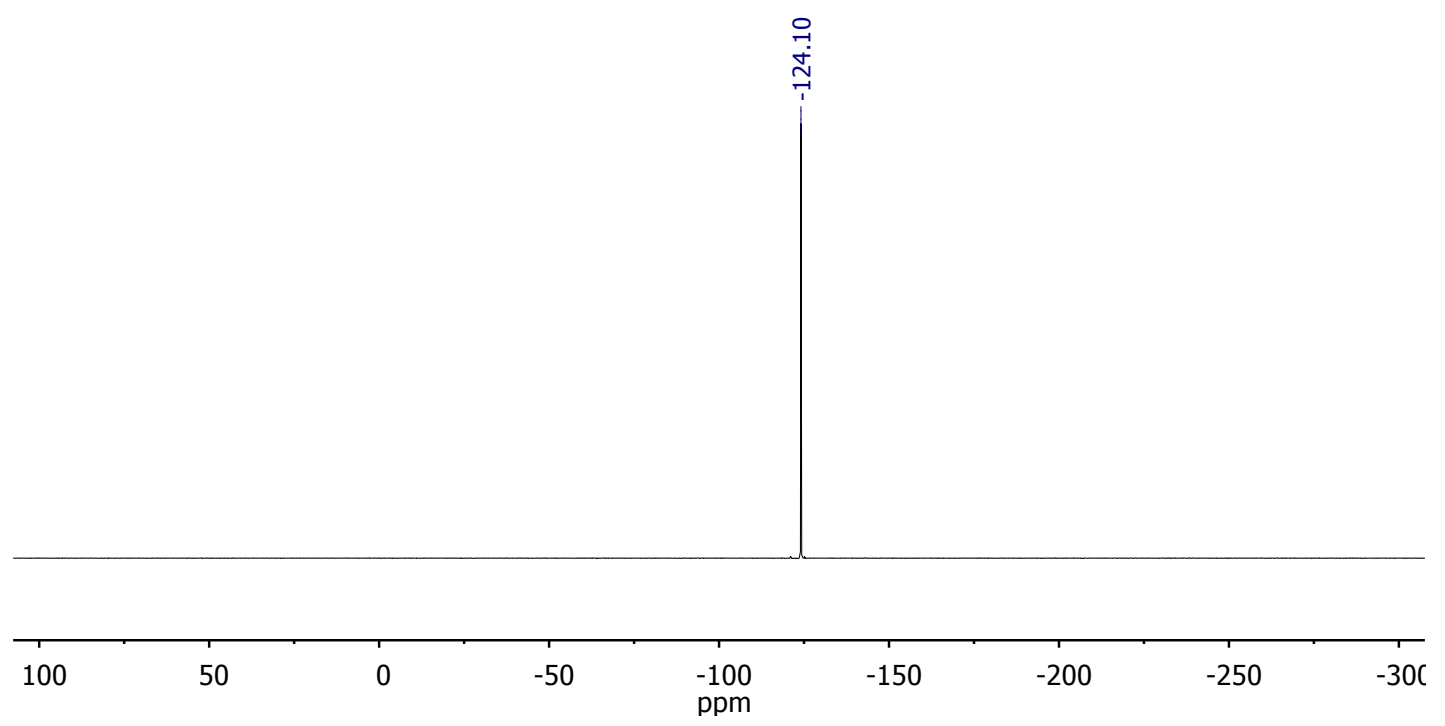


${ }^{13} \mathrm{CNMR}\left(101 \mathrm{MHz}, \mathrm{CDCl}_{3}\right)$

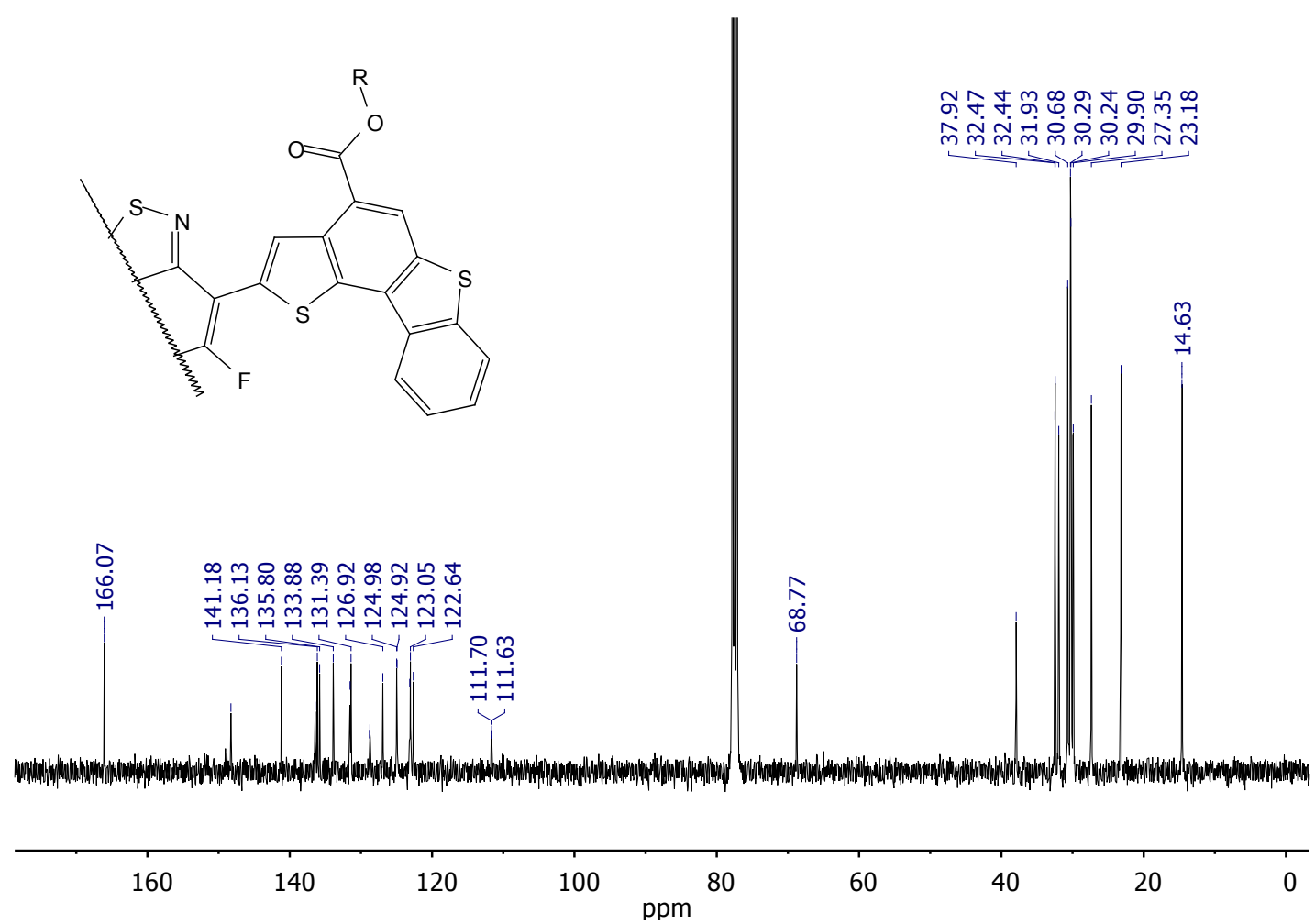

$D E P T$

DEPT compound 25

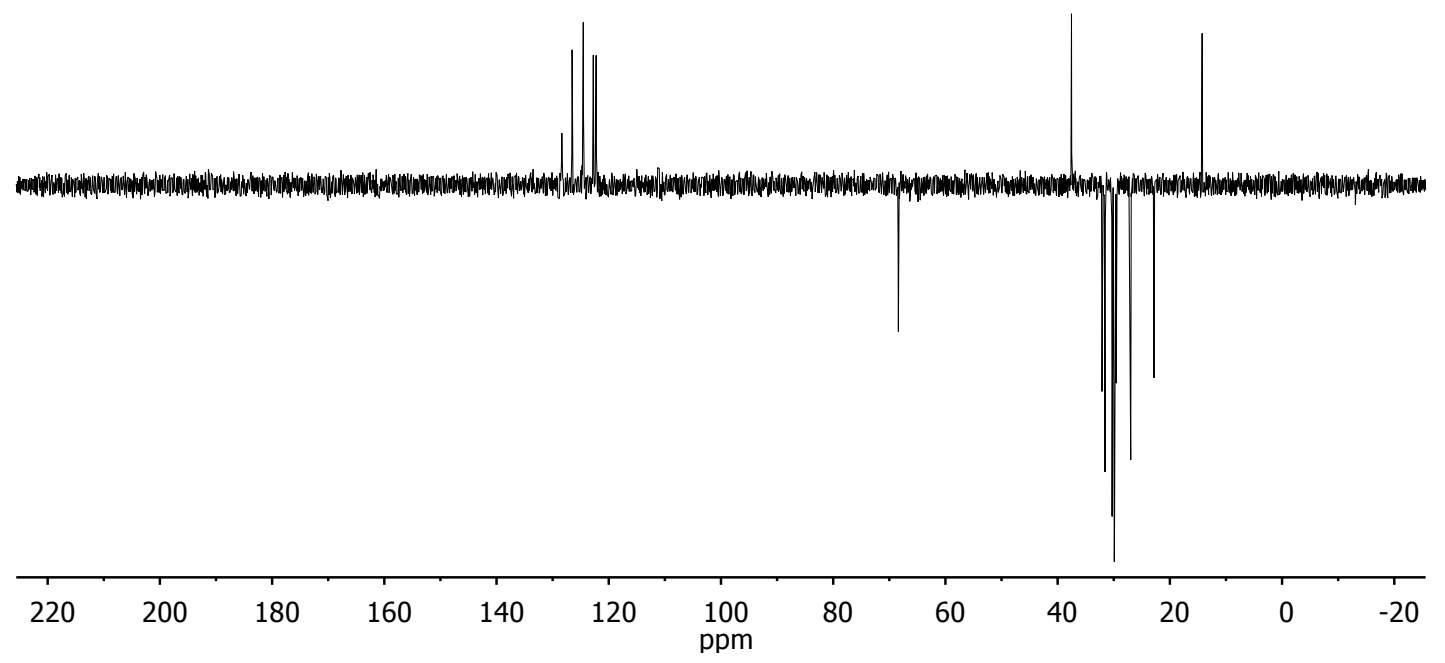


$H M Q C$
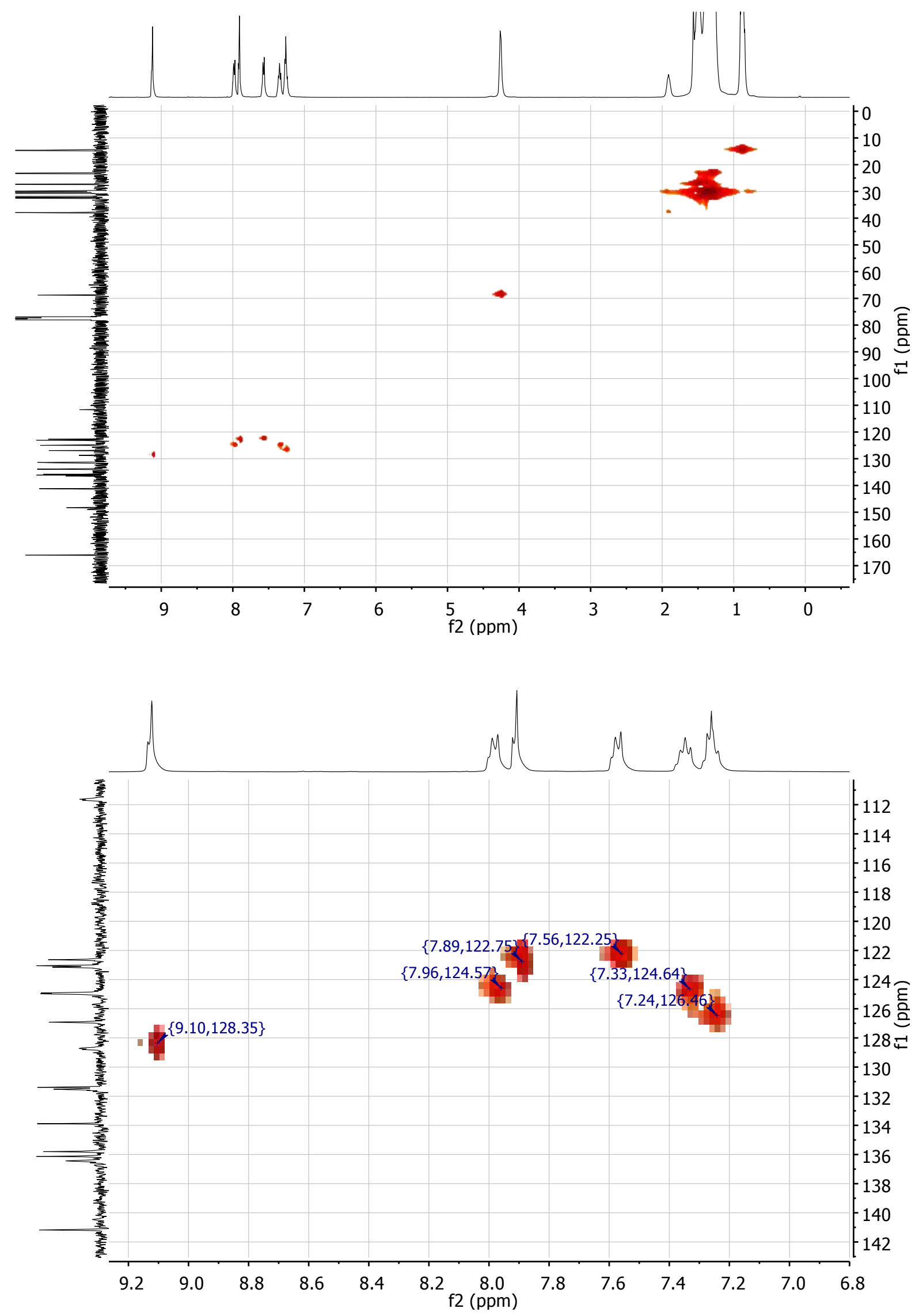

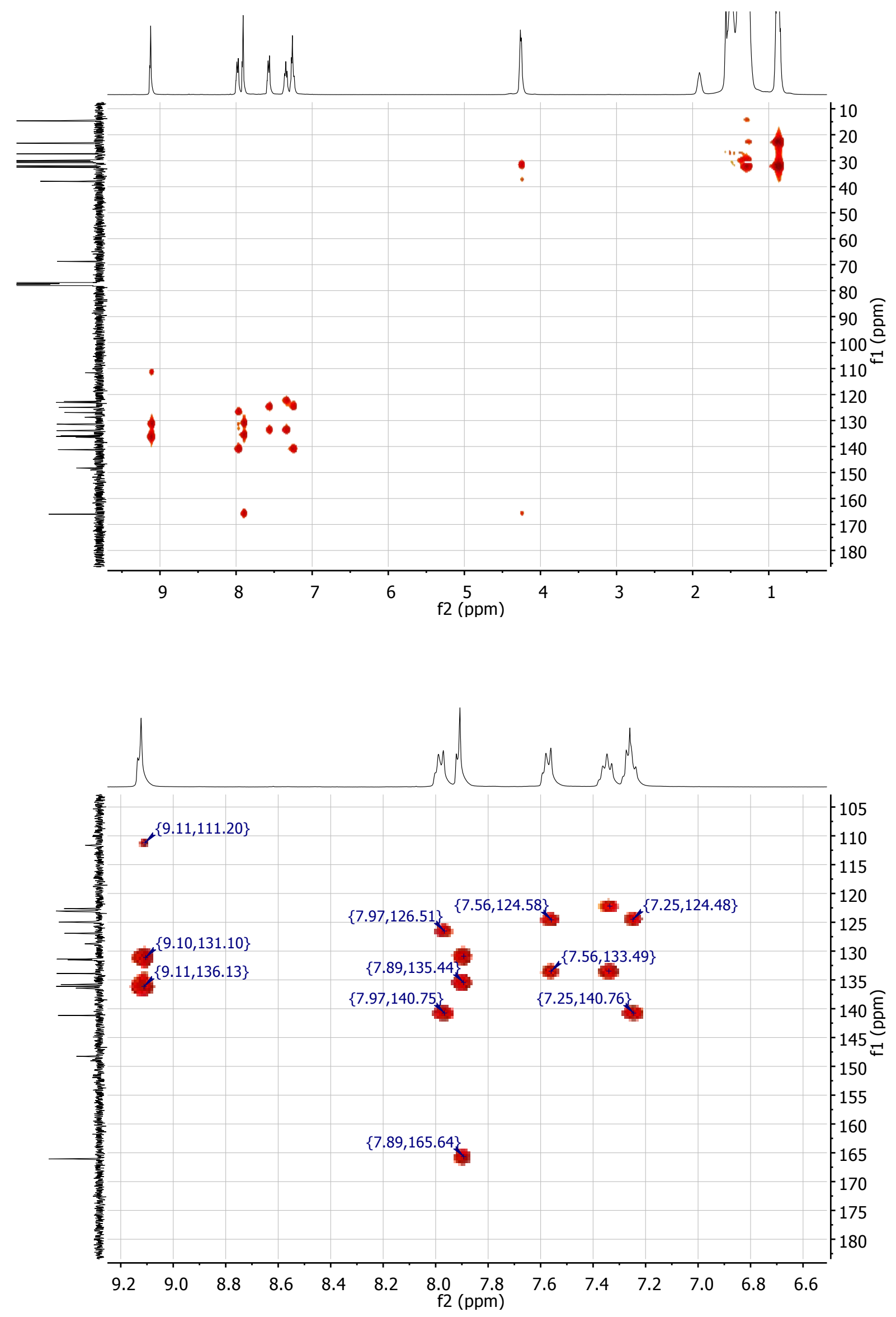


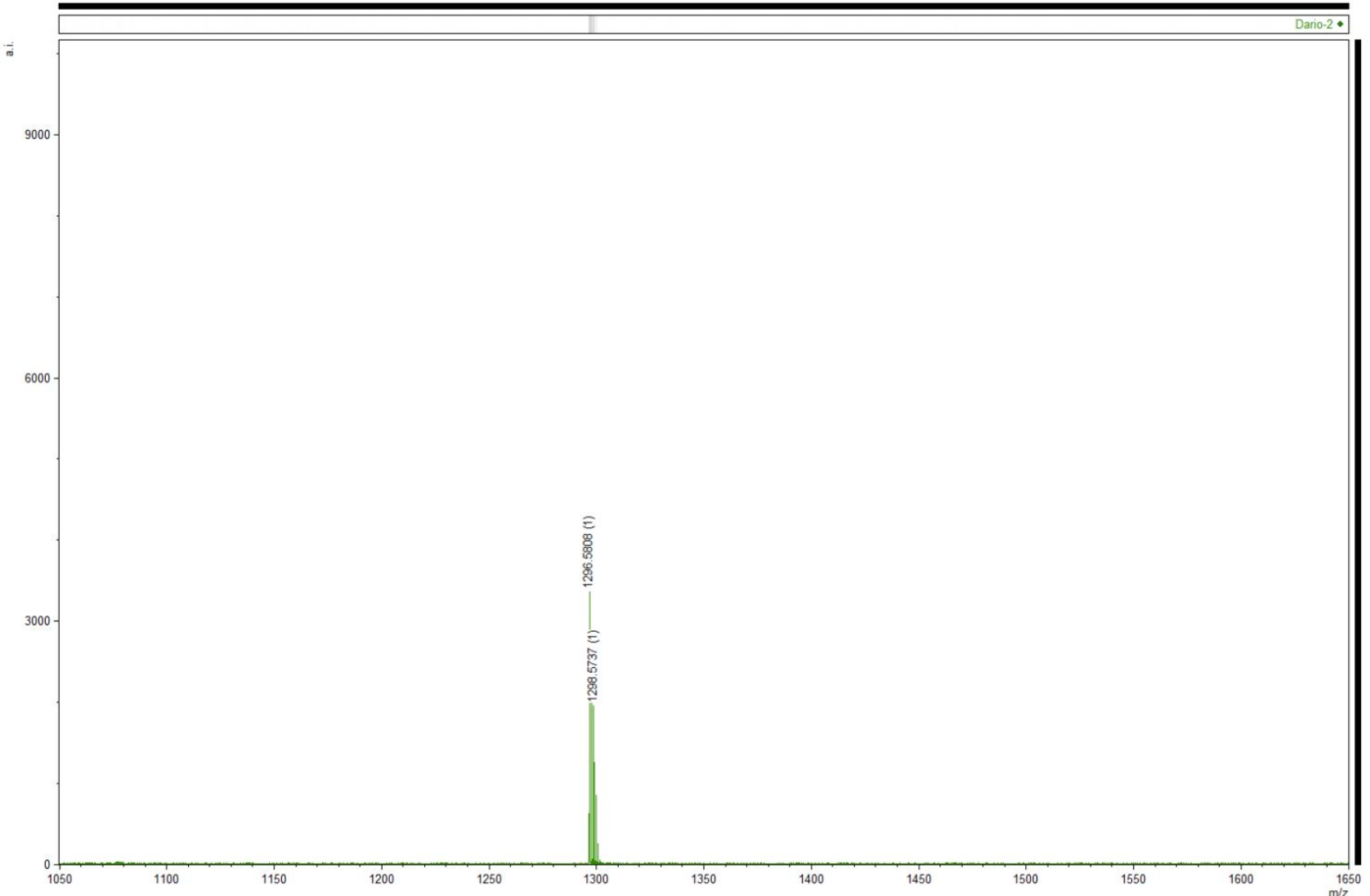


Compound 26

${ }^{1} \mathrm{HNMR}\left(400 \mathrm{MHz}, \mathrm{CDCl}_{3}\right)$

1H NMR compound 26

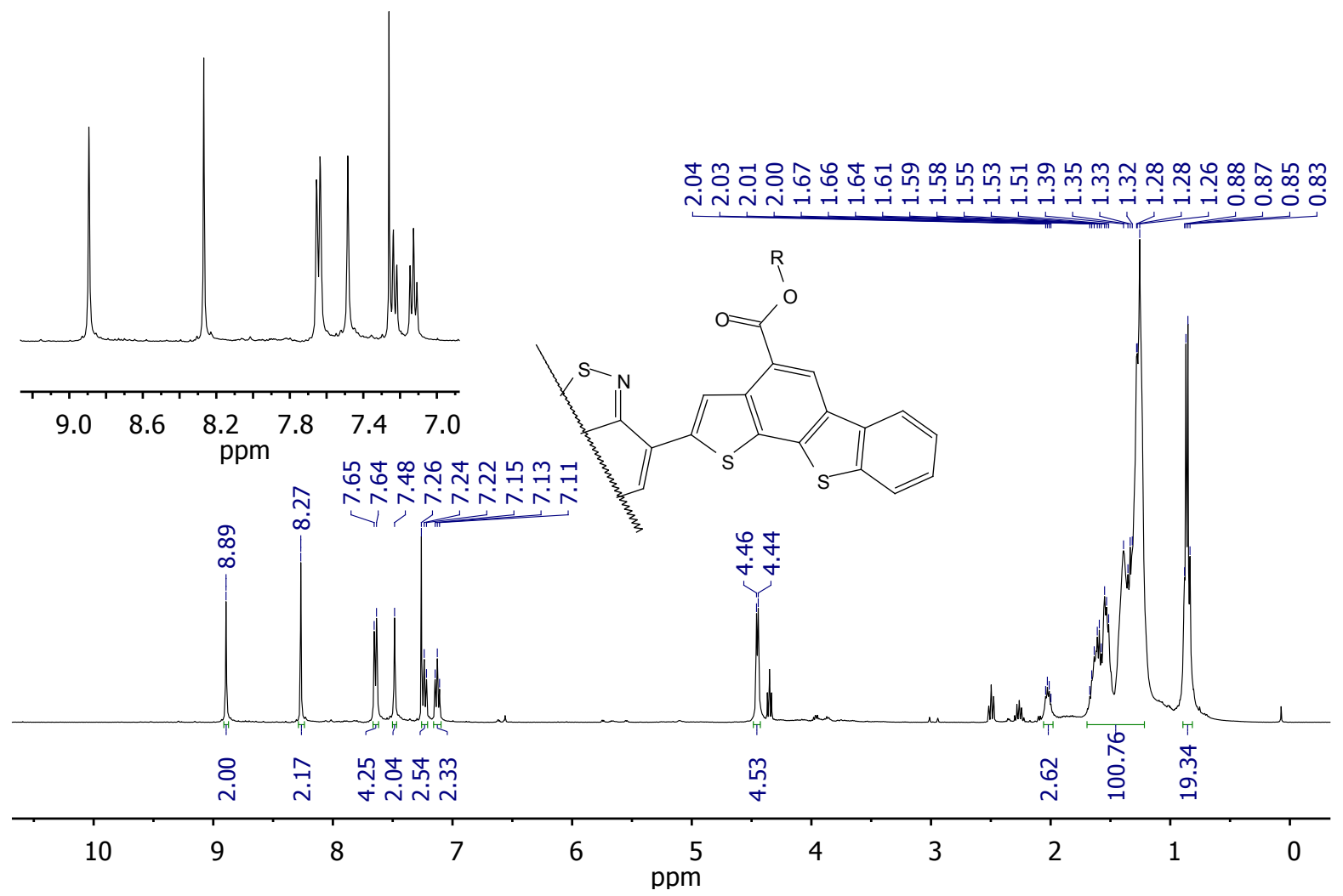

${ }^{13} \mathrm{CNMR}\left(101 \mathrm{MHz}, \mathrm{CDCl}_{3}\right)$

13C NMR compound 26
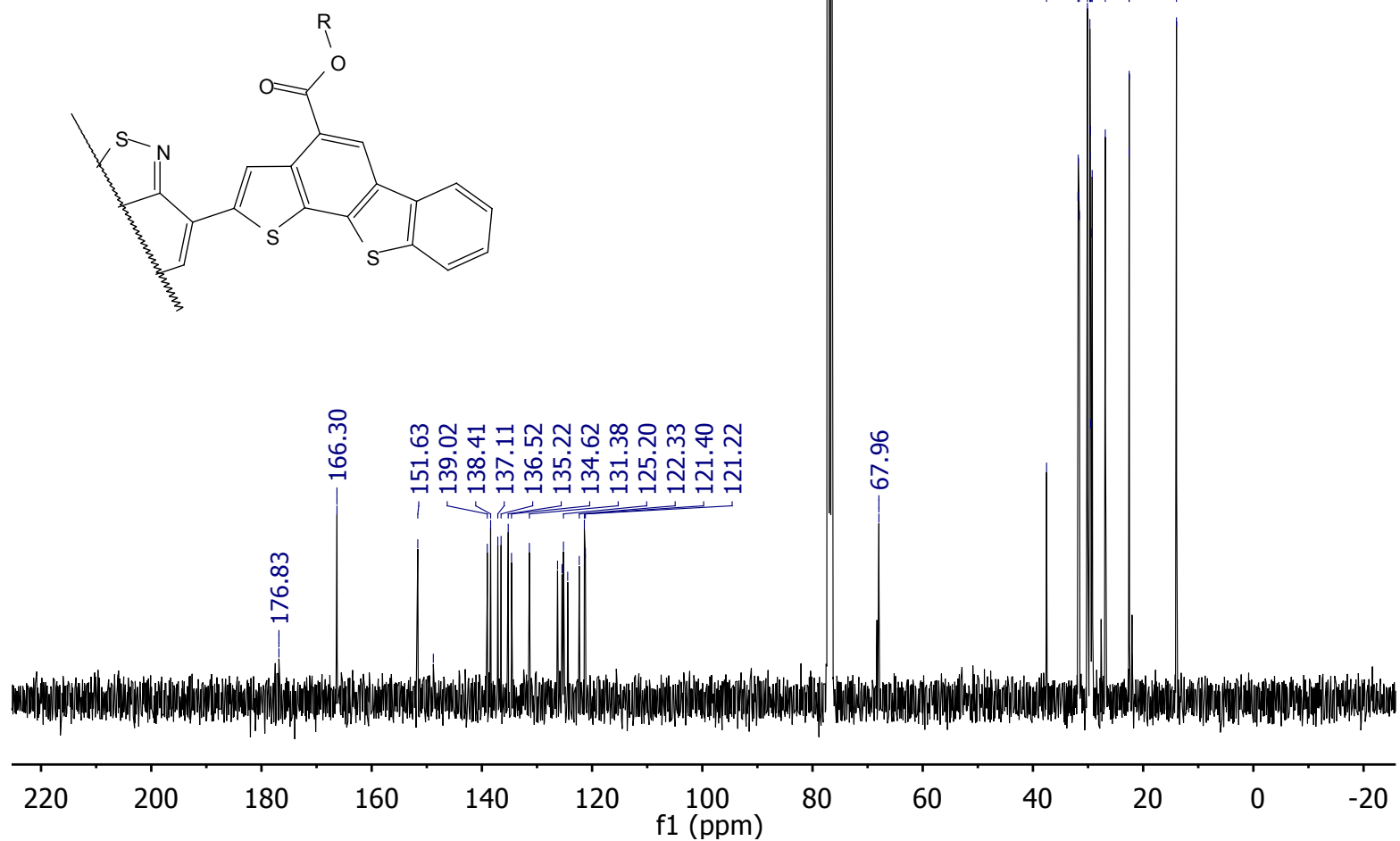


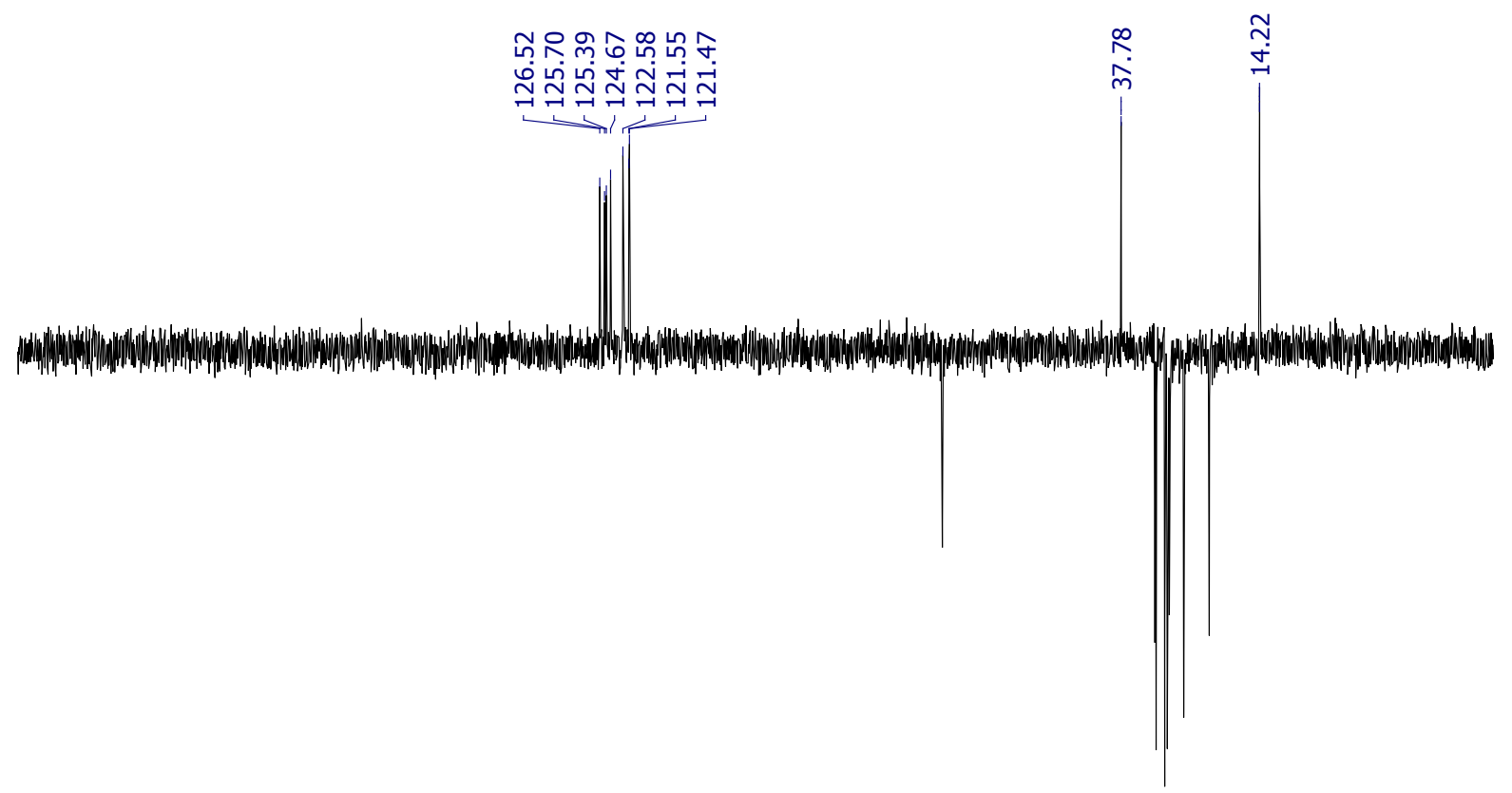

\begin{tabular}{|c|c|c|}
\hline 220 & 200 & 18 \\
\hline
\end{tabular}

$H M Q C$

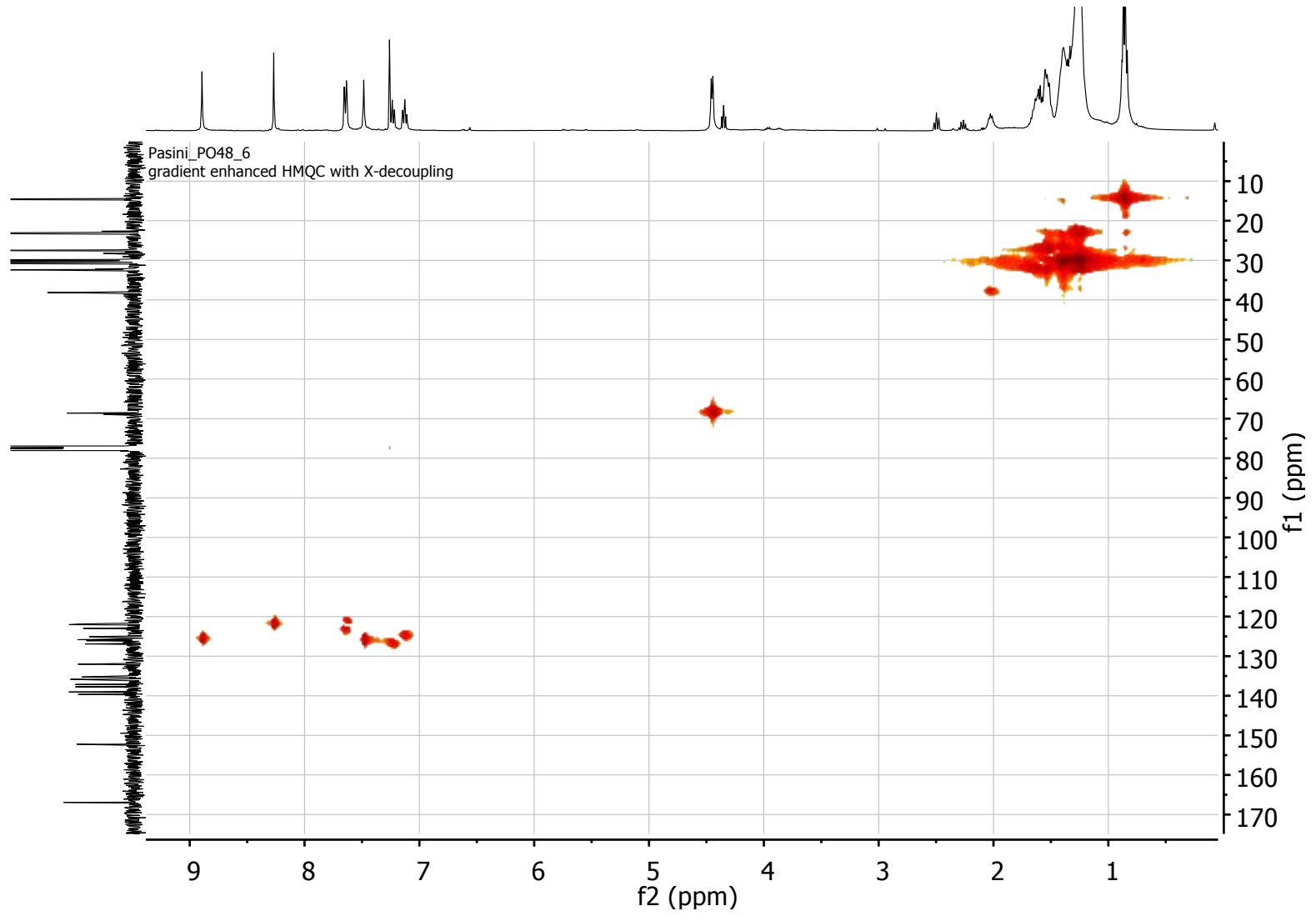




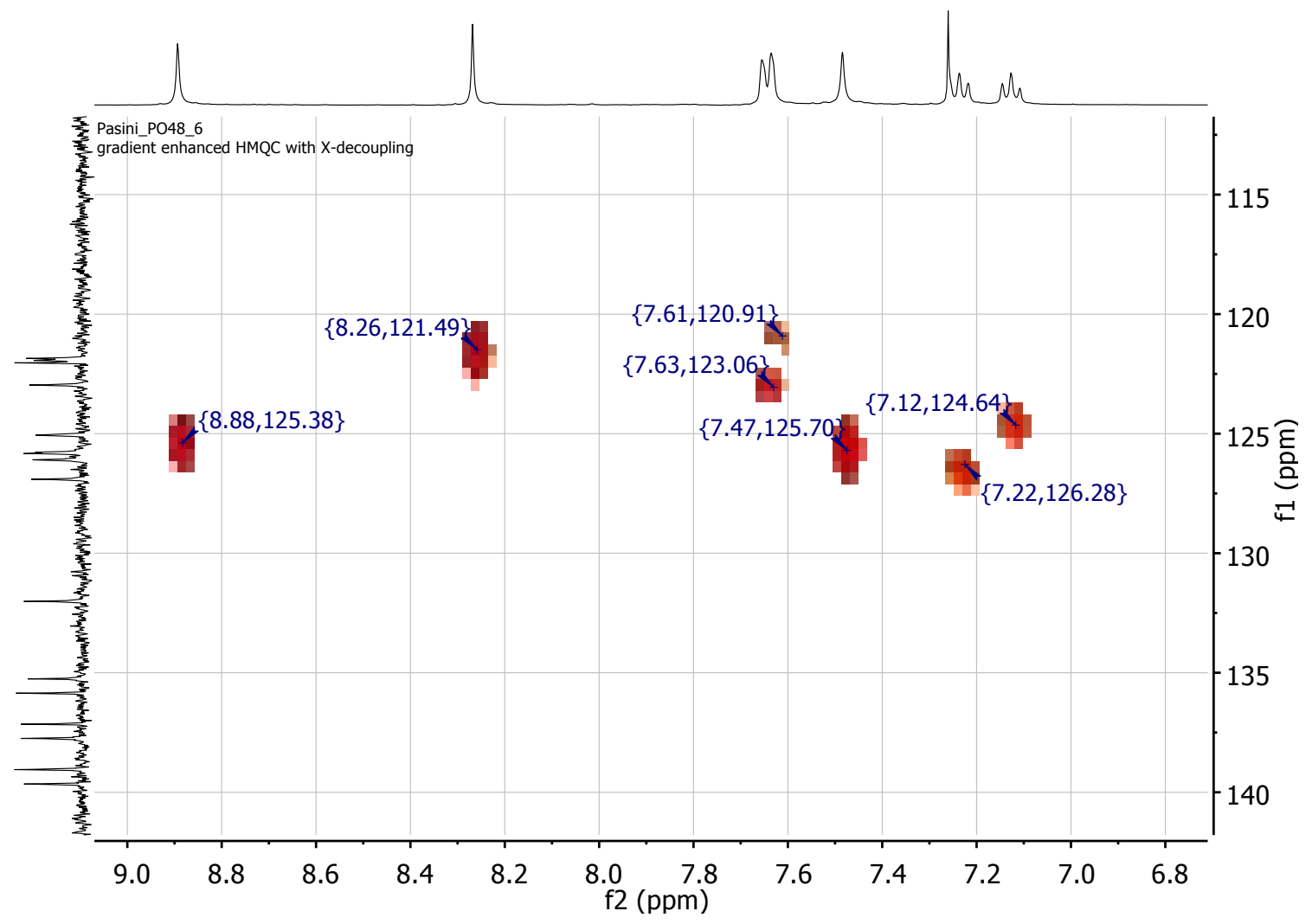

$H M B C$

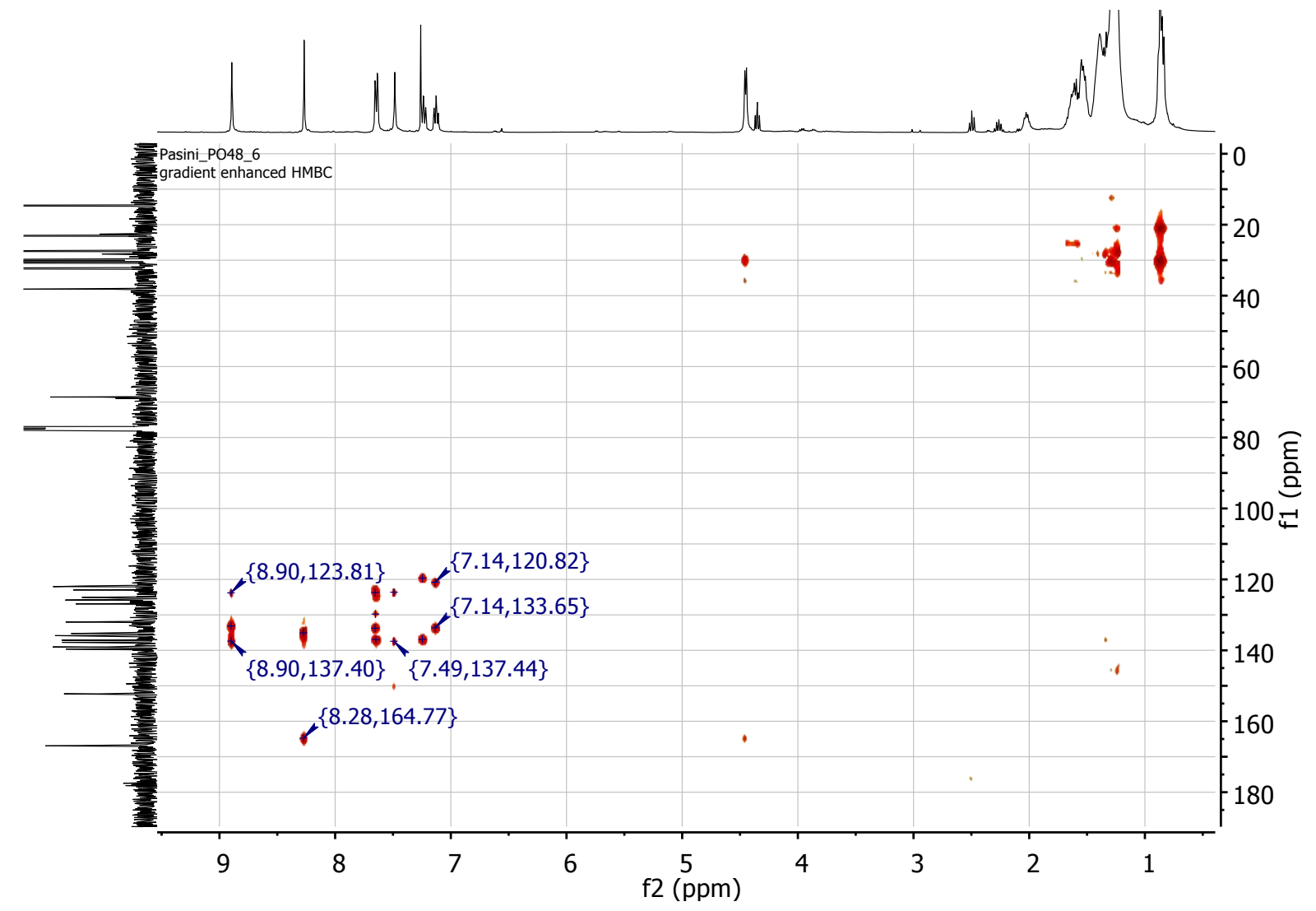




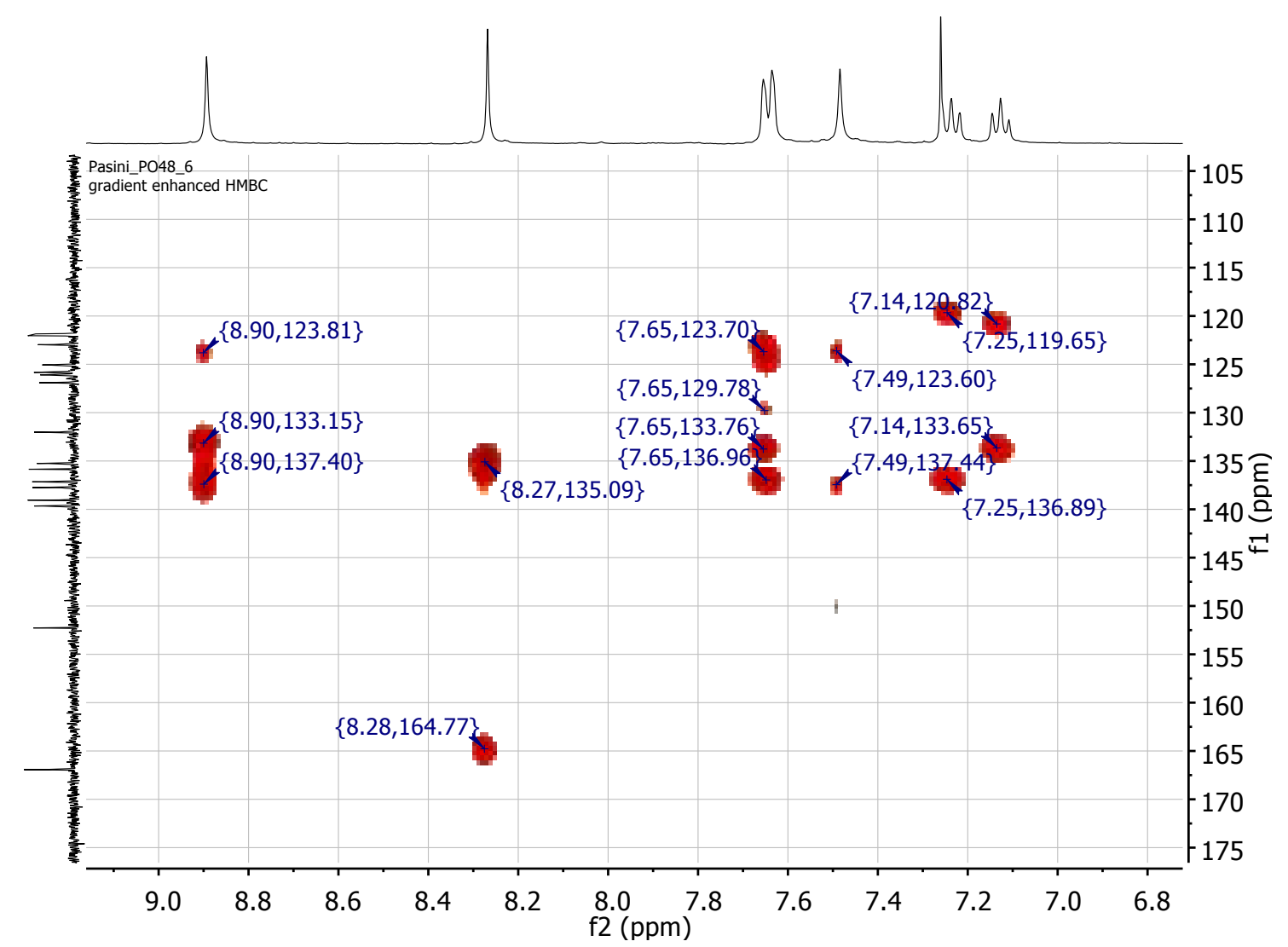

HRMS

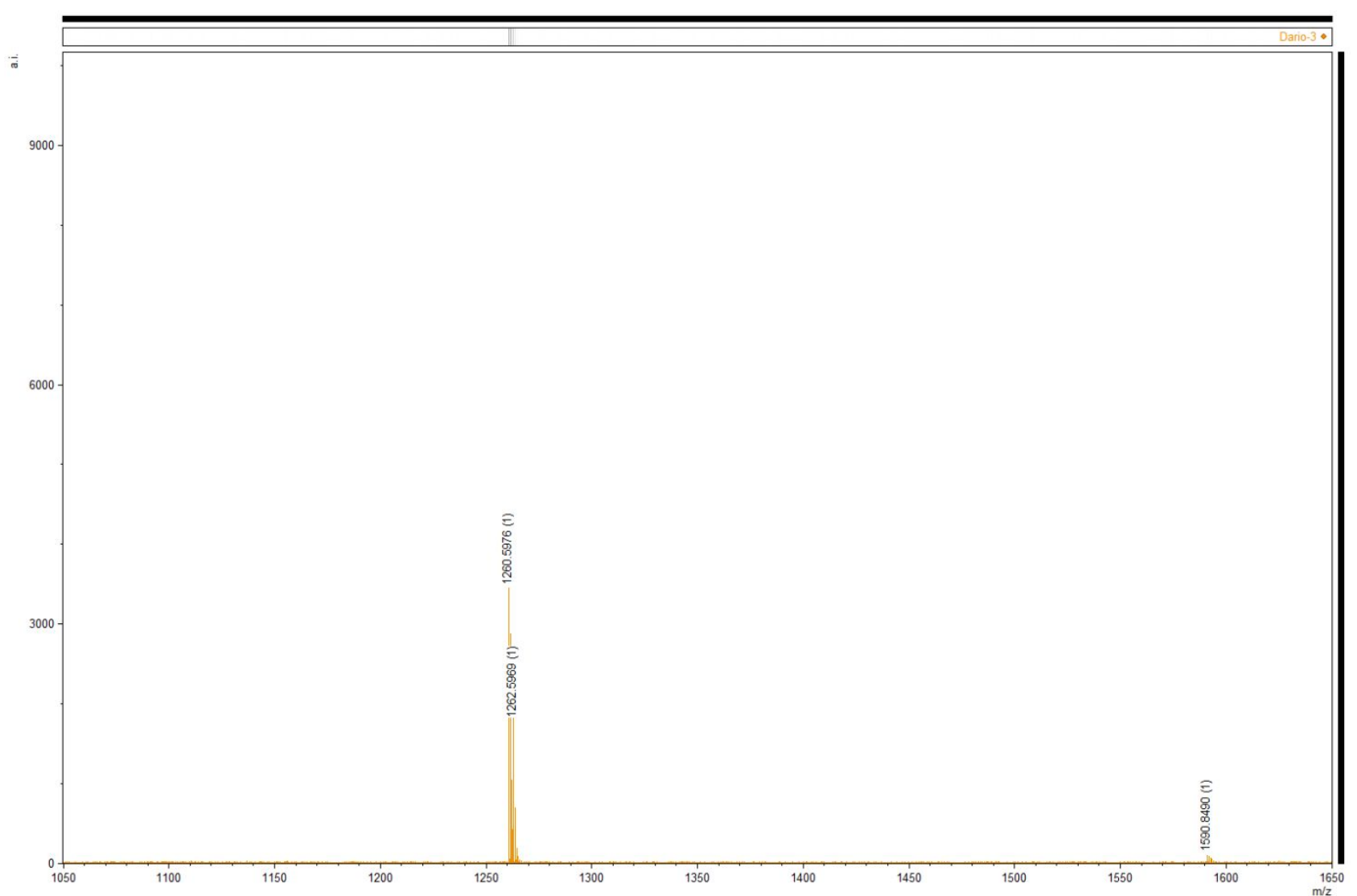


Compound 27

${ }^{1} \mathrm{H} \mathrm{NMR}\left(400 \mathrm{MHz}, \mathrm{CDCl}_{3}\right)$

1HNMR compound 27
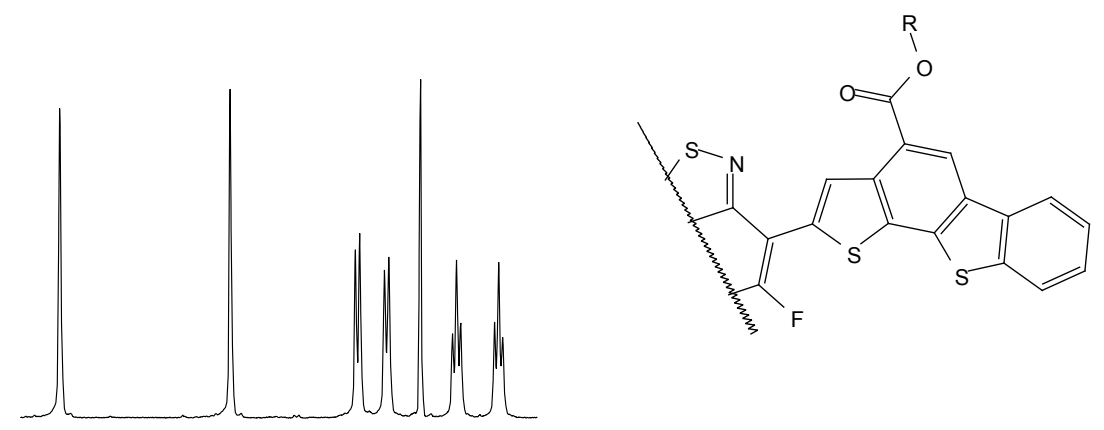

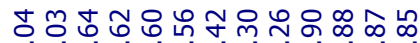

N N
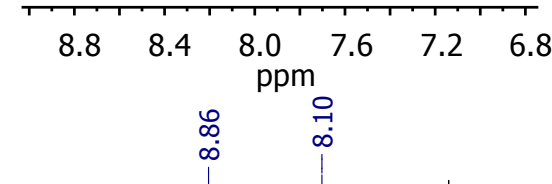

|
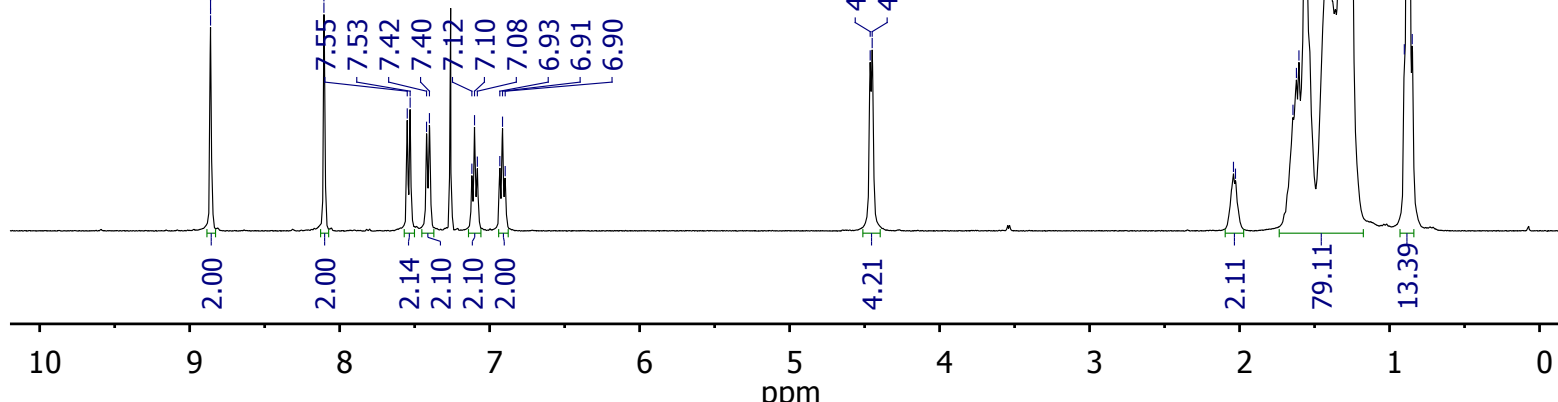

${ }^{19} \mathrm{~F} N M R$

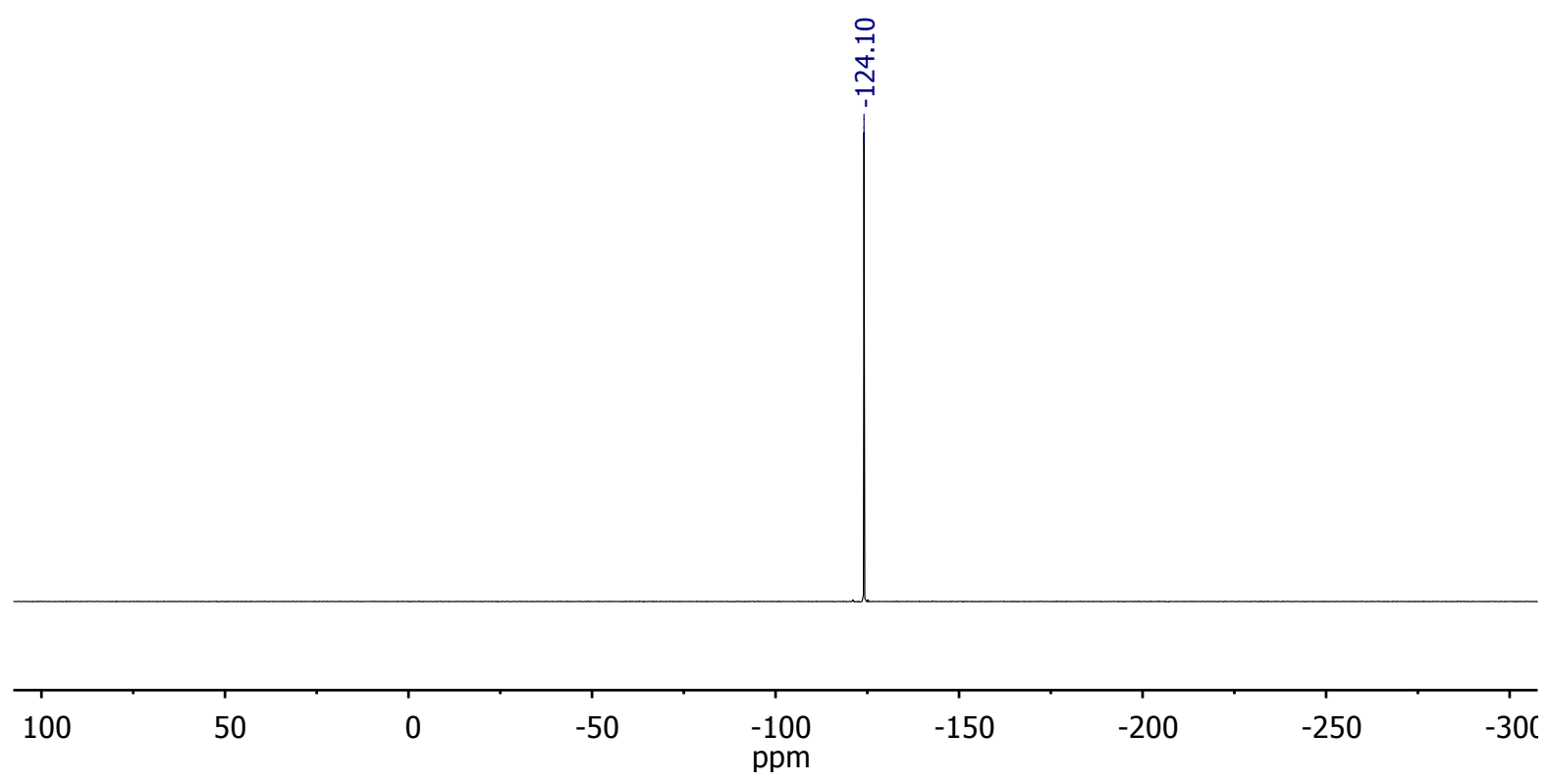




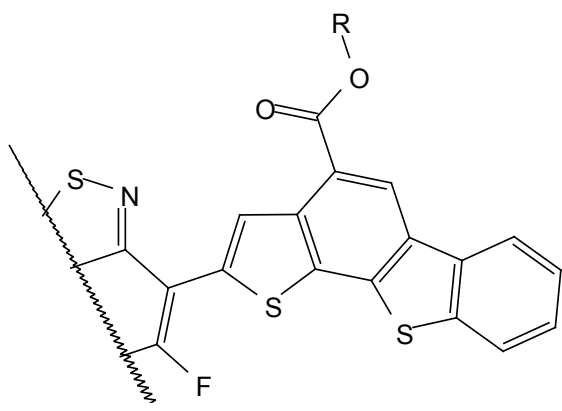

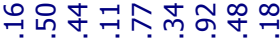

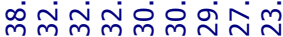
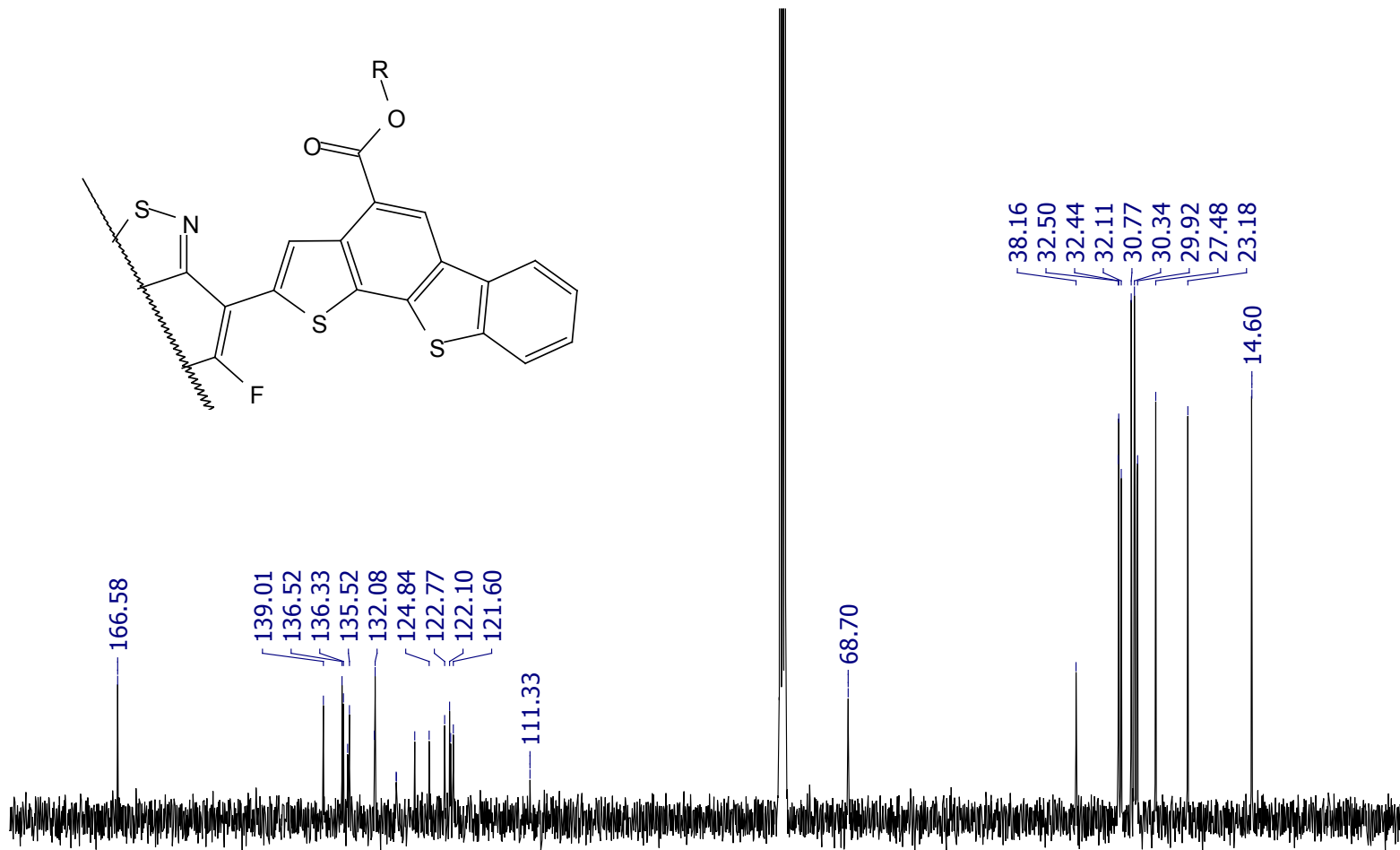

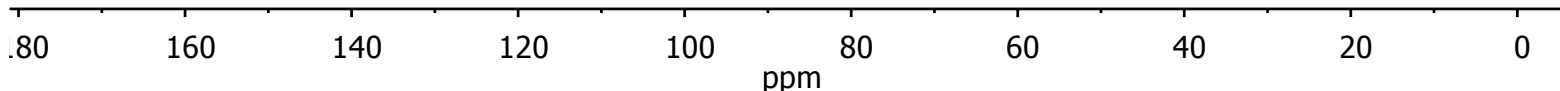

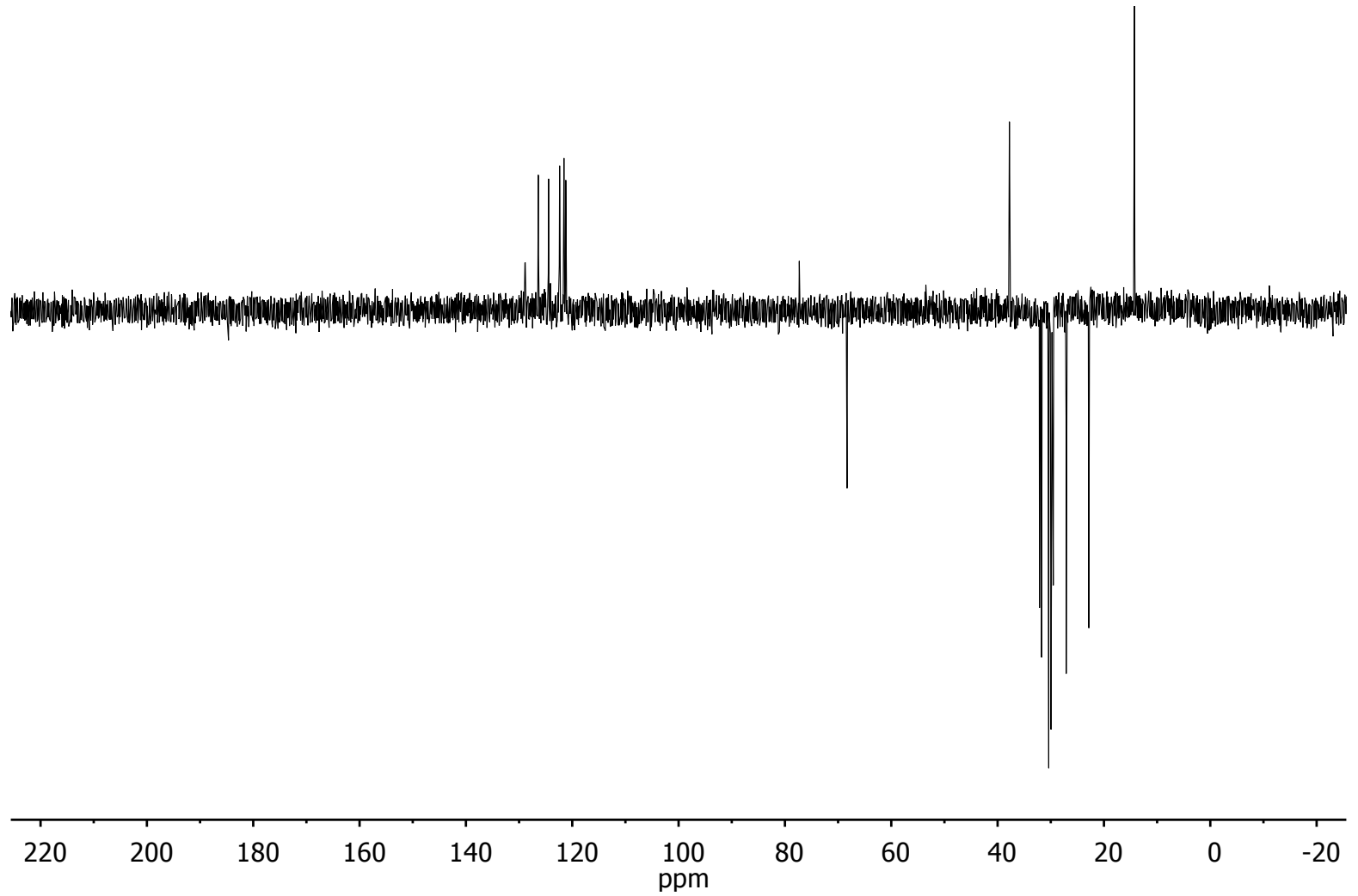



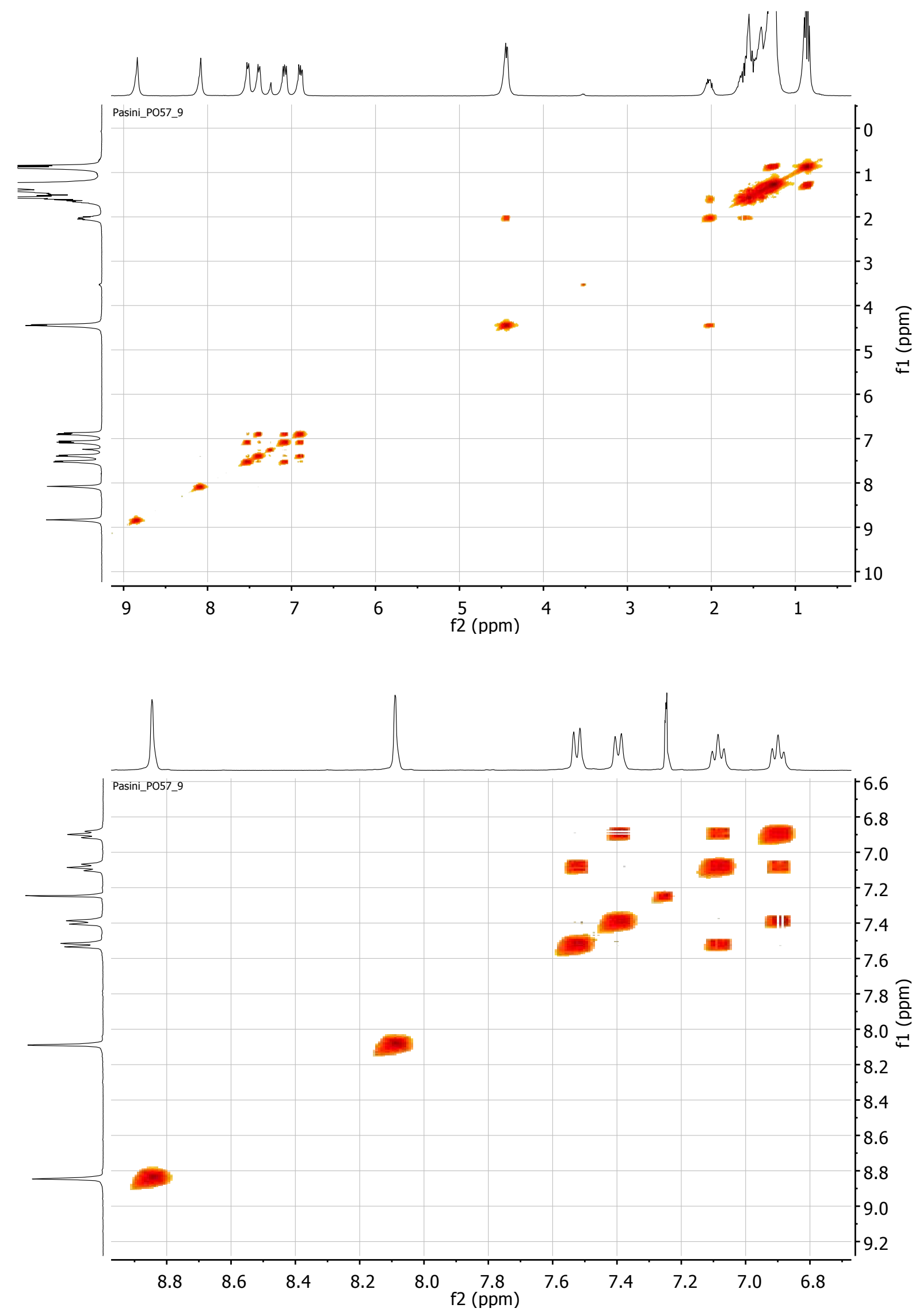

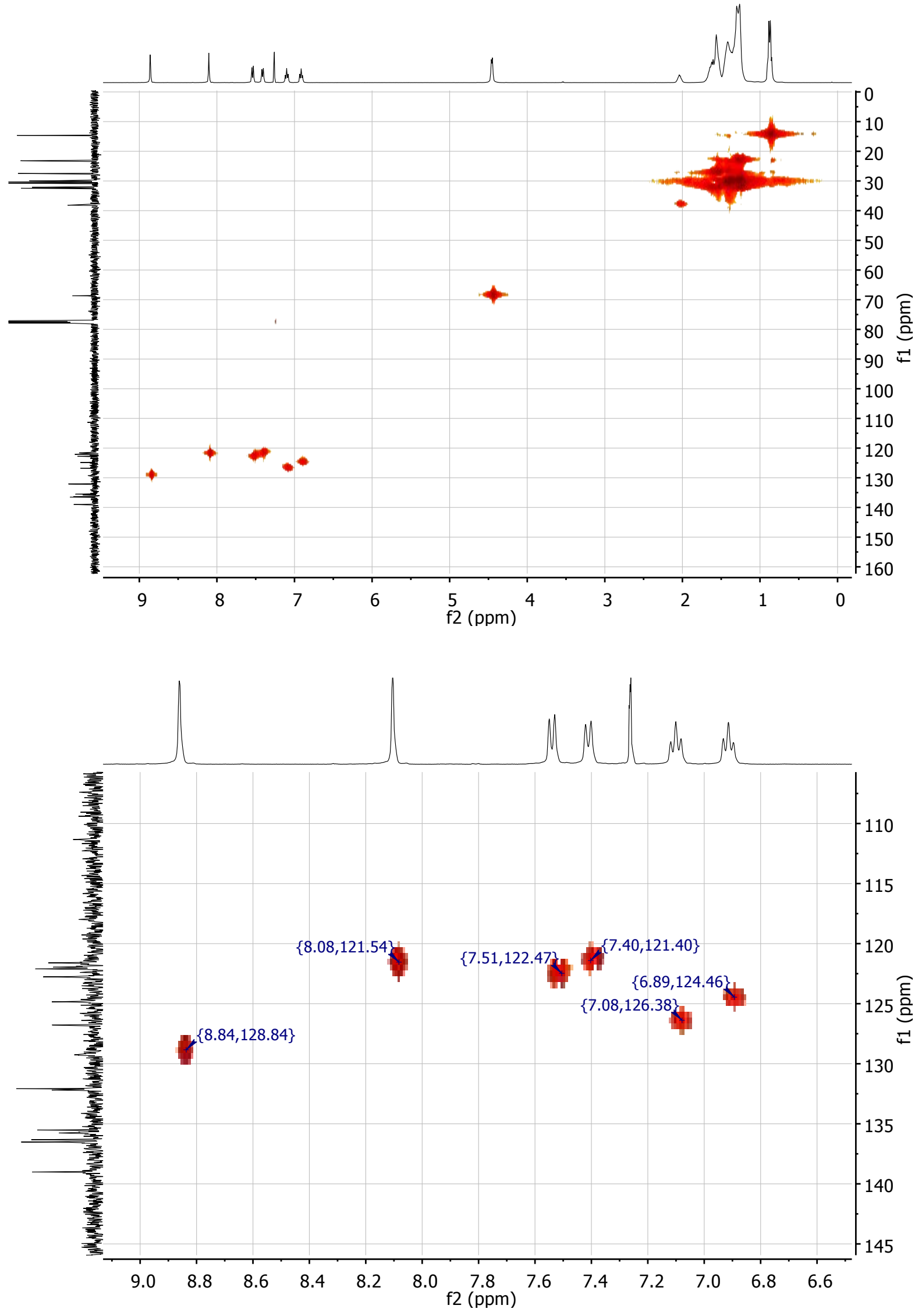

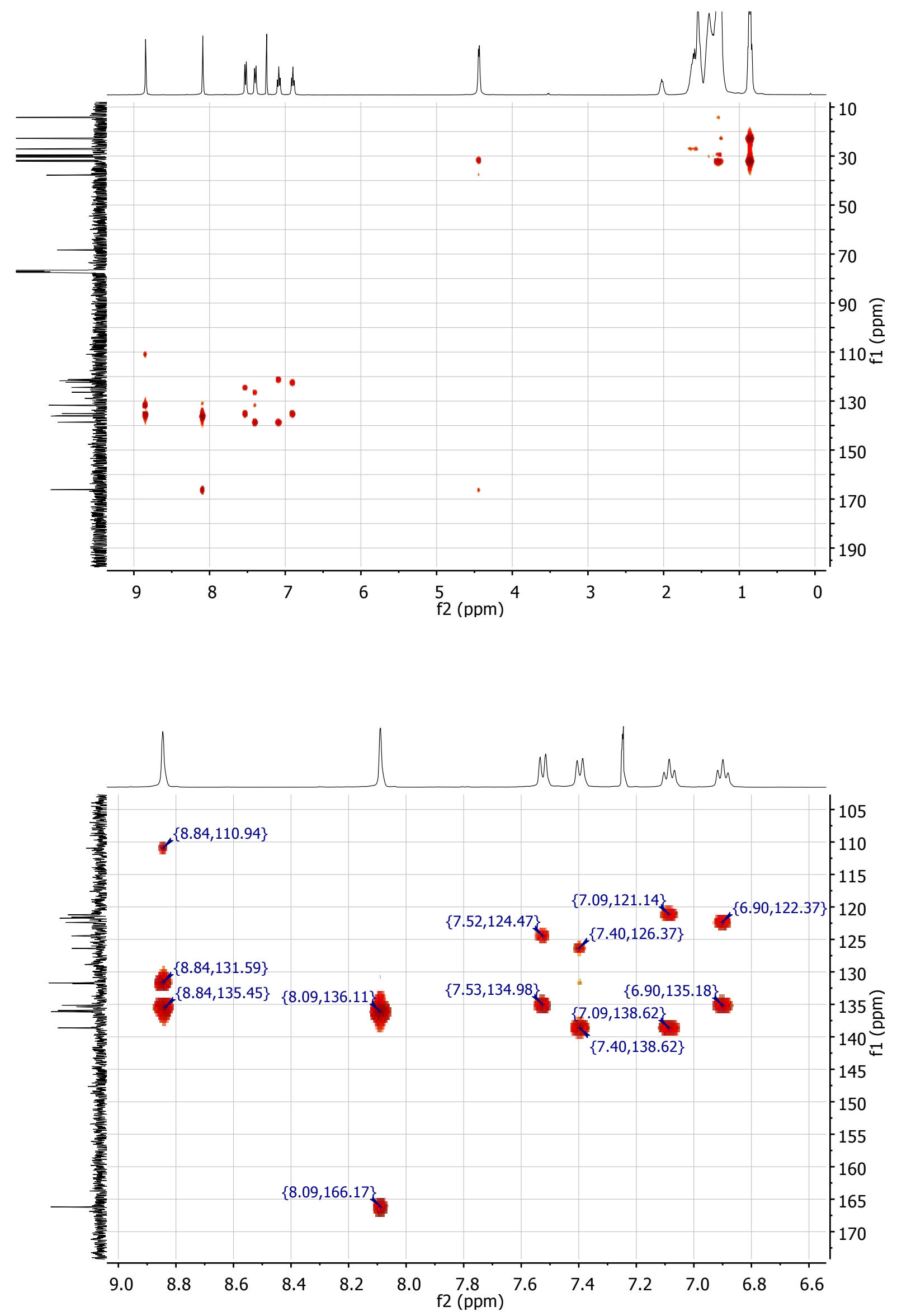


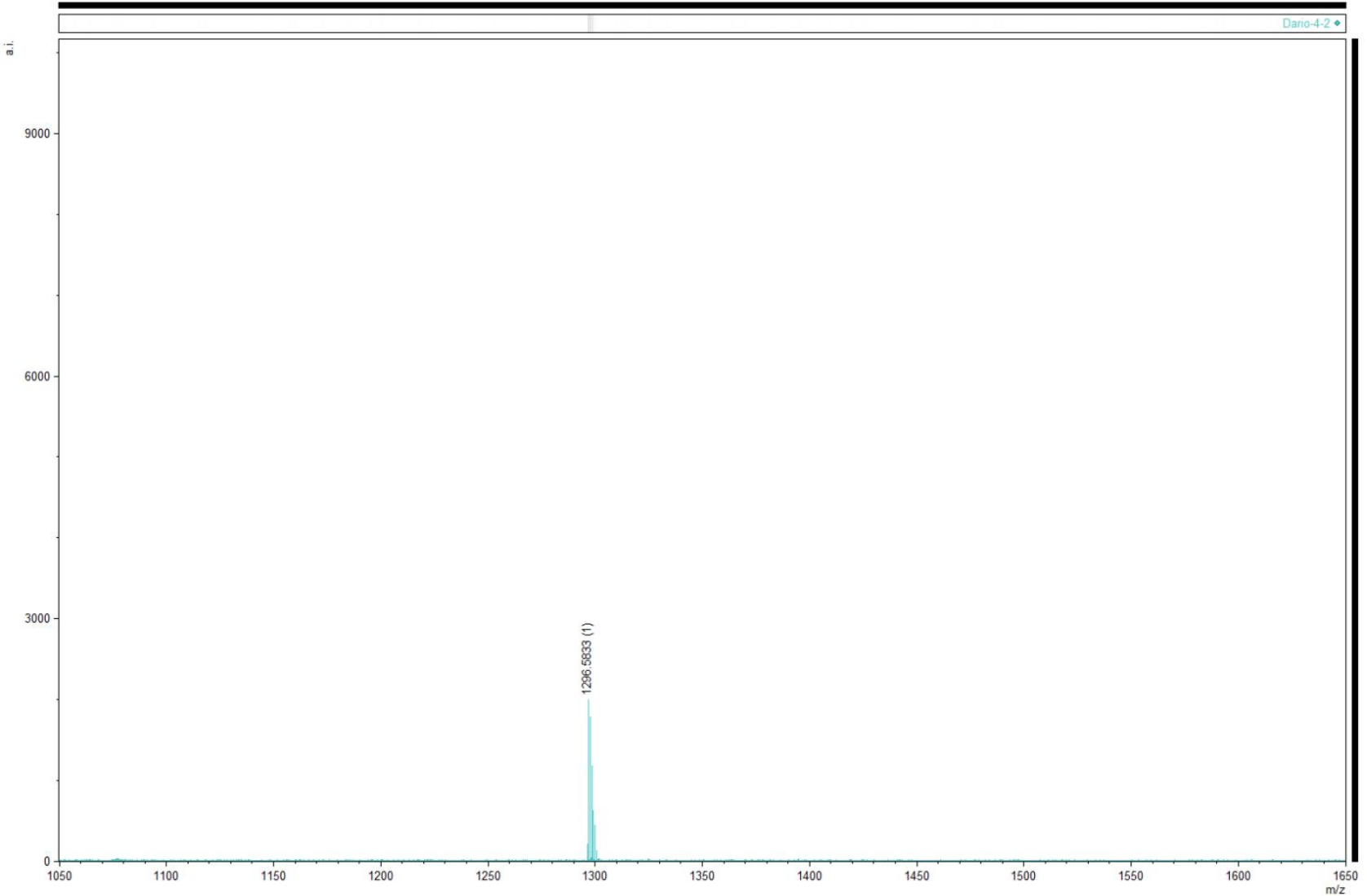

